Supporting Information

\title{
Catalytic Ring-Expansion of Activated Heteroarenes Enabled by Regioselective Dearomatization
}

Jiyoung Kim, and Eun Jeong Yoo*

Department of Applied Chemistry, Kyung Hee University, Yongin 17104, Republic of Korea

Email: ejyoo@khu.ac.kr

\section{Table of contents}

I General Information

II Experimental Procedures $\quad 2$

$\begin{array}{lll}\text { III } & \text { Spectroscopic Data } & 6\end{array}$

IV X-ray Crystal Data (3a) $\quad 14$

$\begin{array}{lll}\text { V Reference } & 15\end{array}$

VI Copies of Spectral Data of Compounds Obtained in this Study 16

Acknowledgment We acknowledge the Korea Basic Science Institute (KBSI) for the HRMS analysis. 


\section{General Information}

Unless otherwise stated, all commercial reagents and solvents were used without additional purification. Analytical thin layer chromatography (TLC) was performed on Merck pre-coated silica gel 60 F254 plates. Visualization on TLC was achieved by use of UV light $(254 \mathrm{~nm})$. Flash column chromatography was undertaken on silica gel (Merck Kiesel gel 60 F254 230-400 mesh). ${ }^{1} \mathrm{H}$ NMR were recorded on JEOL $(400 \mathrm{MHz})$ and JEOL $(300 \mathrm{MHz})$. Chemical shifts were quoted in parts per million (ppm) referenced to the appropriate solvent peak or $0.0 \mathrm{ppm}$ for tetramethylsilane. The following abbreviations were used to describe peak splitting patterns when appropriate: $b r=b r o a d, s=\operatorname{singlet}, d=$ doublet, $t=$ triplet, $q=$ quartet, $m=$ multiplet. Coupling constants, $J$, were reported in hertz unit $(\mathrm{Hz}) \cdot{ }^{1{ }^{3} \mathrm{C} N M R}$ were recorded on JEOL $(100 \mathrm{MHz})$ and JEOL $(75 \mathrm{MHz})$. There were fully decoupled by broad band proton decoupling. Chemical shifts were reported in ppm referenced to the center line of a triplet at $77.0 \mathrm{ppm}$ of chloroform- $d$. Infrared spectra were recorded on JASCO FT/IR-4700 and FT/IR-4200 FT-IR spectrometer. High resolution mass spectra were obtained from the Korea Basic Science Institute (Daegu) and the Organic Chemistry Research Center in Sogang University by electrospray ionization (ESI) apparatus using Q-TOF (quadrupole-time of flight) mass spectrometry. Single crystal x-ray diffraction data was collected on a Bruker D8 QUEST coated with Paraton- $N$ oil under a stream of $\mathrm{N}_{2}$ (g) at $173 \mathrm{~K}$. The diffraction data were integrated, scaled, and reduced by using the Bruker APEX3 software. The crystal structure(s) were solved by SHELX structure solution program and refined by fullmatrix least-squares calculations with the SHELXL.

\section{Experimental Procedures}

\section{II-1. Experimental Procedure for the Synthesis of Starting Materials}

\section{A. Procedure for the Synthesis of N-Aromatic Zwitterions}
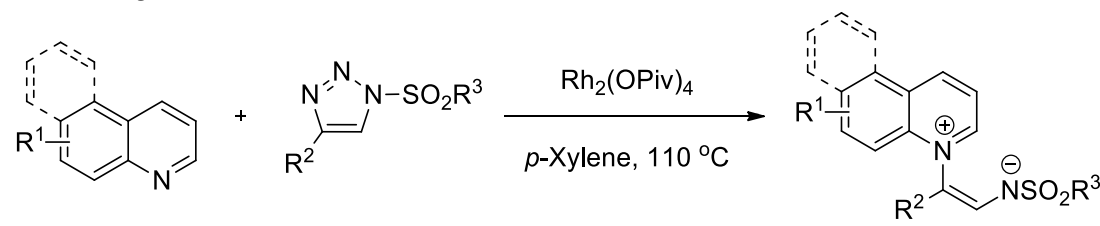

$\mathrm{N}$-aromatic zwitterion

$\mathrm{N}$-aromatic zwitterions (1) were prepared according to the reported procedure. ${ }^{\mathrm{S} 1}$ To a test tube with a triangular-shaped stir bar were added 4-phenyl-1-ptoluenesulfonyl-1,2,3-triazole (2.0 equiv), and $\mathrm{Rh}_{2}(\mathrm{OPiv})_{4}(4.0 \mathrm{~mol} \%)$, and $p$-xylene $(2.0 \mathrm{~mL})$ under $\mathrm{N}_{2}$ atmosphere. To this stirred reaction mixture was added $\mathrm{N}$-heteroaromatic compound $(0.2 \mathrm{mmol})$ and the reaction mixture was stirred at $110{ }^{\circ} \mathrm{C}$ for the indicated time. The reaction mixture was then cooled to room temperature and concentrated under reduced pressure to remove the solvent and the residue was purified by chromatography on silica gel to give the desired $\mathrm{N}$-aromatic zwitterion.

\section{B. Procedure for the Synthesis of Acyl Diazomethanes}

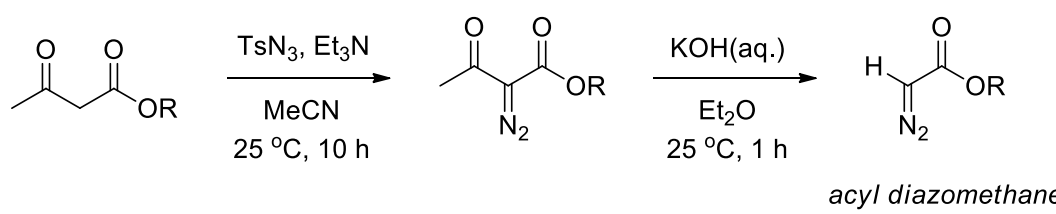

The acyl diazomethanes (2) were prepared according to the reported procedure. ${ }^{\mathrm{S} 2}$ To a solution of 
acetoacetates $(10.0 \mathrm{mmol})$ in acetonitrile $(12 \mathrm{~mL})$ was added triethylamine (1.3 equiv). The solution was cooled in an ice bath and a solution of tosyl azide (1.1 equiv) in acetonitrile (12 mL) was added slowly. The reaction mixture was warmed to $25^{\circ} \mathrm{C}$. After stirring for $10 \mathrm{~h}$, the solvent was removed under reduced pressure. The residue was dissolved in diethyl ether $(60 \mathrm{~mL})$ and washed with $5 \%$ aqueous $\mathrm{KOH}$ solution. To a solution of the crude diazo acetoacetate in diethyl ether was added $5 \% \mathrm{KOH}(50$ $\mathrm{mL}$ ), and the reaction mixture was stirred for $1 \mathrm{~h}$. The organic phase was separated, dried over $\mathrm{Na}_{2} \mathrm{SO}_{4}$, and concentrated under reduced pressure. Purification by vacuum distillation provided the desired product.

\section{Procedure for the Synthesis of Silver(I) Catalysts}

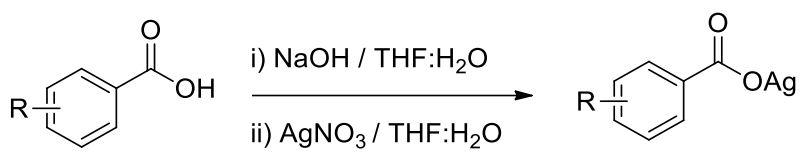

The silver(I) catalysts were prepared according to the reported procedure. ${ }^{\mathrm{S} 3}$ To a round bottom flask was charged with benzoic acids $(3.0 \mathrm{mmol})$ and THF $(5 \mathrm{~mL})$. The mixture was stirred till all the acid was dissolved. A solution of $\mathrm{NaOH}$ (1.0 equiv) in water $(2.5 \mathrm{~mL}$ ) was added to the above solution, and the reaction mixture was stirred at $25^{\circ} \mathrm{C}$ for $10 \mathrm{~min}$. Then, the solvent was removed and the residue was suspended in THF $(5 \mathrm{~mL})$. A solution of $\mathrm{AgNO}_{3}(0.83$ equiv) in water $(2.5 \mathrm{~mL})$ was slowly added to the above suspension and stirred at $25^{\circ} \mathrm{C}$ for $10 \mathrm{~min}$. Afterward, the solvent was evaporated on a rotary. The residue was washed with water and dried under reduced pressure to obtain the desired catalyst.

\section{II-2. Experimental Procedure for the Silver-Catalyzed Ring-Expansion Reactions}

\section{A. Experimental Procedure for the Optimization (Table 1 and Table S1)}

To a flame dried test tube with a triangular-shaped stir bar were added quinolinium zwitterion (1a, 0.2 $\mathrm{mmol}$ ), ethyl diazoacetate (2a, 3.0 equiv), indicated catalyst ( $5 \mathrm{~mol} \%$ ), indicated additive (1.3 equiv), and indicated solvent $(4.0 \mathrm{~mL})$ under $\mathrm{N}_{2}$ atmospheric conditions. The reaction mixture stirred at $35^{\circ} \mathrm{C}$ for $12 \mathrm{~h}$. After cooling the reaction mixture, it was filtered through a pad of celite and then washed with $\mathrm{CH}_{2} \mathrm{Cl}_{2}(10 \mathrm{~mL} \times 3)$. The combined organic layer was concentrated under reduced pressure. The organic residue was purified by chromatography on silica gel deactivated with $5 \% \mathrm{Et}_{3} \mathrm{~N}$ to give the desired product 3.

Table S1. Optimization of the Ring Expansion Reaction ${ }^{a}$

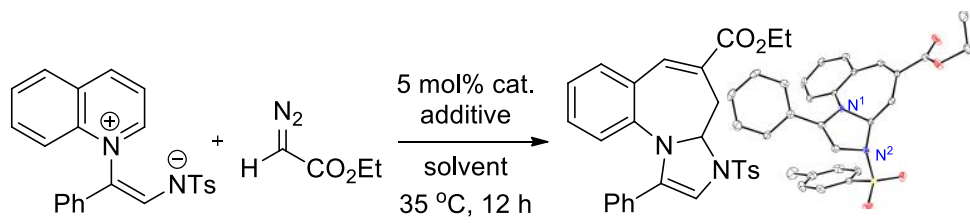

$1 \mathrm{a}$

2a

$3 a$

$\begin{array}{ccccc}\text { entry } & \text { catalyst } & \text { additive } & \text { solvent } & \text { yield }(\%)^{b} \\ 1 & \mathrm{AgOTf} & - & \text { THF } & 58 \\ 2 & \mathrm{Sc}(\mathrm{OTf})_{3} & - & \text { THF } & <1 \\ 3 & \mathrm{Al}(\mathrm{OTf})_{3} & - & \text { THF } & <1 \\ 4^{c} & \mathrm{AgNO}_{3} & - & \text { THF } & 31\end{array}$




$\begin{array}{ccccc}5 & \mathrm{AgOBz} & - & \mathrm{THF} & 49 \\ 6 & \mathrm{Ag}\left[\mathrm{O}_{2} \mathrm{C}\left(3-\mathrm{ClC}_{6} \mathrm{H}_{4}\right)\right] & - & \mathrm{THF} & 34 \\ 7 & \mathrm{Ag}\left[\mathrm{O}_{2} \mathrm{C}\left(3,5-\left(\mathrm{NO}_{2}\right)_{2} \mathrm{C}_{6} \mathrm{H}_{3}\right)\right] & - & \mathrm{THF} & 83 \\ 8 & \mathrm{Ag}\left[\mathrm{O}_{2} \mathrm{C}\left(3,5-\left(\mathrm{NO}_{2}\right)_{2} \mathrm{C}_{6} \mathrm{H}_{3}\right)\right] & - & \text { Toluene } & 21 \\ 9 & \mathrm{Ag}\left[\mathrm{O}_{2} \mathrm{C}\left(3,5-\left(\mathrm{NO}_{2}\right)_{2} \mathrm{C}_{6} \mathrm{H}_{3}\right)\right] & - & (2-\mathrm{Me}) \mathrm{THF} & 59 \\ 10 & \mathrm{Ag}\left[\mathrm{O}_{2} \mathrm{C}\left(3,5-\left(\mathrm{NO}_{2}\right)_{2} \mathrm{C}_{6} \mathrm{H}_{3}\right)\right] & - & 1,4-\text { dioxane } & 58 \\ 11 & \mathrm{Ag}\left[\mathrm{O}_{2} \mathrm{C}\left(3,5-\left(\mathrm{NO}_{2}\right)_{2} \mathrm{C}_{6} \mathrm{H}_{3}\right)\right] & - & \text { DMF } & 31 \\ 12 & \mathrm{Cu}\left[\mathrm{O}_{2} \mathrm{C}\left(3,5-\left(\mathrm{NO}_{2}\right)_{2} \mathrm{C}_{6} \mathrm{H}_{3}\right)\right] & - & \text { THF } & <1 \\ 13 & & \mathrm{~K}\left[\mathrm{O}_{2} \mathrm{C}\left(3,5-\left(\mathrm{NO}_{2}\right)_{2} \mathrm{C}_{6} \mathrm{H}_{3}\right)\right] & \mathrm{THF} & <1 \\ 14 & - & \mathrm{DBU} & \mathrm{THF} & <1 \\ 15 & - & \mathrm{TEA} & \mathrm{THF} & <1 \\ 16 & - & \mathrm{NaH} & \mathrm{THF} & <1 \\ 17^{d} & - & \text { LDA } & \text { THF } & <1\end{array}$

${ }^{a}$ Reaction conditions: 1a $(0.2 \mathrm{mmol}), 2 a(3.0$ equiv), catalyst ( $5 \mathrm{~mol} \%)$, additive (1.3 equiv), and solvent $(4 \mathrm{~mL})$ at $35^{\circ} \mathrm{C}$ for $12 \mathrm{~h}$. ${ }^{b}$ Isolated yields. ${ }^{c} 10$ mol\% of catalyst was used. ${ }^{d} 3.0$ equiv of HMPA were additionally added.

\section{B. General Procedure for the Silver-Catalyzed Ring-Expansion (Scheme 2)}<smiles>[R]C(=C[N+](=O)[O-])[n+]1cccc2c3ncccc3[R1]cc21</smiles>

1

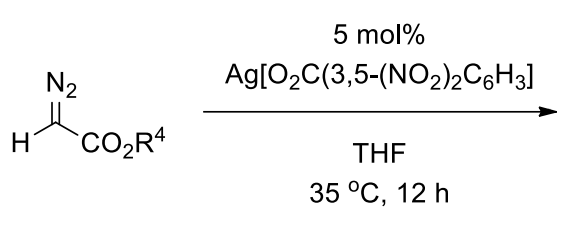

2<smiles>[R]OC1=Cc2c(cc[R1]3ccccc32)N2C([R])=C[N+]([R20])=CC2C1</smiles>

3

To a flame dried test tube with a triangular-shaped stir bar were added quinolinium zwitterion $(\mathbf{1}, 0.2$ $\mathrm{mmol}$ ), acyl diazomethanes (2, 3.0 equiv), silver 3,5-dinitrobenzoate ( $5 \mathrm{~mol} \%)$, and THF $(4.0 \mathrm{~mL})$ under $\mathrm{N}_{2}$ atmospheric conditions. The reaction mixture stirred at $35{ }^{\circ} \mathrm{C}$ for $12 \mathrm{~h}$. After cooling the reaction mixture, it was filtered through a pad of celite and then washed with $\mathrm{CH}_{2} \mathrm{Cl}_{2}(10 \mathrm{~mL} \times 3)$. The combined organic layer was concentrated under reduced pressure. The organic residue was purified by chromatography on silica gel deactivated with $5 \% \mathrm{Et}_{3} \mathrm{~N}$ to give the desired product 3.

\section{II-3. Procedure for Synthetic Applications (Scheme 3)}

\section{A. Procedure for Scale-up Reaction}

To a flame dried round bottom flask with a stirring bar were added quinolinium zwitterion (1a, $1.0 \mathrm{~g}, 2.5$ $\mathrm{mmol}$ ), ethyl diazoacetate (2a, 3.0 equiv), silver 3,5-dinitrobenzoate (5 mol\%), and THF (50 mL) under $\mathrm{N}_{2}$ atmospheric conditions. The reaction mixture stirred at $35{ }^{\circ} \mathrm{C}$ for $12 \mathrm{~h}$. After cooling the reaction mixture, it was filtered through a pad of celite and then washed with $\mathrm{CH}_{2} \mathrm{Cl}_{2}(10 \mathrm{~mL} \times 3)$. The organic residue was purified by chromatography on silica gel deactivated with $5 \% \mathrm{Et}_{3} \mathrm{~N}$ to give the desired product $3 \mathrm{a}(75 \%, 912.4 \mathrm{mg})$.

\section{B. Procedure for the Detosylation of Compound 3a to Afford Compound 4}

To a flame dried test tube with a triangular-shaped stir bar were added compound $3 \mathbf{a}(0.1 \mathrm{mmol}), \mathrm{K}_{2} \mathrm{CO}_{3}$ 
(1.5 equiv), and DMF $(2.0 \mathrm{~mL})$ under $\mathrm{N}_{2}$ atmospheric conditions. The reaction mixture was stirred at $50^{\circ} \mathrm{C}$. After $21 \mathrm{~h}$, the reaction mixture was quenched with $\mathrm{H}_{2} \mathrm{O}$. The mixture was extracted with EtOAc $(10 \mathrm{~mL} \times 3)$, and the organic phase was dried over $\mathrm{MgSO}_{4}$ and concentrated under reduced pressure. The organic residue was purified by chromatography on silica gel to give the desired product 4 .

\section{Procedure for Selective Reduction of Compound 4 to Afford Compound 5}

To a flame dried test tube with a triangular-shape stir bar were added compound $4(0.1 \mathrm{mmol}), \mathrm{Pd} / \mathrm{C}$ (20 mol\%), and $\mathrm{EtOH}(2.5 \mathrm{~mL})$. The reaction vessel was closed, evacuated three times and backfilled by $\mathrm{H}_{2}$-balloon. The reaction mixture was stirred at $35{ }^{\circ} \mathrm{C}$ under $\mathrm{H}_{2}$-balloon pressure for $24 \mathrm{~h}$. After cooling the reaction mixture, it was filtered through a pad of celite and then washed with $\mathrm{CH}_{2} \mathrm{Cl}_{2}(10 \mathrm{~mL}$ $x 3$ ). The combined organic layer was concentrated under reduced pressure. The organic residue was purified by chromatography on silica gel to give the desired product 5 .

\section{Procedure for Selective Reduction of Compound 4 to Afford Compound 6}

To a flame dried test tube with a triangular-shape stir bar were added compound $\mathbf{4}(0.2 \mathrm{mmol})$, and THF $(3.0 \mathrm{~mL})$ under $\mathrm{N}_{2}$ atmospheric conditions and DIBAL-H (2.5 equiv) was slowly added at $0{ }^{\circ} \mathrm{C}$. The reaction mixture was stirred at $35^{\circ} \mathrm{C}$. After $8 \mathrm{~h}$, the reaction mixture was quenched with $1 \mathrm{~N} \mathrm{HCl}$. The mixture was extracted with EtOAc $(10 \mathrm{~mL} \times 3)$, and the organic phase was dried over $\mathrm{MgSO}_{4}$ and concentrated under reduced pressure. The organic residue was purified by chromatography on silica gel to give the desired product 6 .

\section{E. Procedure for the Appel Reaction of Compound 6 to Afford Compound 7}

To a flame dried test tube with a triangular-shape stir bar were added compound $6(0.1 \mathrm{mmol}), \mathrm{PPh}_{3}$ (1.5 equiv), $\mathrm{CCl}_{4}(1.3 \mathrm{~mL})$, and $\mathrm{CH}_{2} \mathrm{Cl}_{2}(1.3 \mathrm{~mL})$ under $\mathrm{N}_{2}$ atmospheric conditions. The reaction mixture was refluxed for $19 \mathrm{~h}$. After cooling the reaction mixture, it was filtered through a pad of celite and then washed with $\mathrm{CH}_{2} \mathrm{Cl}_{2}(10 \mathrm{~mL} \times 3)$. The combined organic layer was concentrated under reduced pressure. The organic residue was purified by chromatography on silica gel to give the desired product 7 .

\section{II-4. Mechanistic Investigations (Scheme 4)}

\section{A. The Reaction of 2-D-labeled Quinolinium Zwitterion (1a-D) and Ethyl Diazoacetate (2a)}

To a flame dried test tube with a triangular-shaped stir bar were added 2-D-labeled quinolinium zwitterion (1a-D, $0.2 \mathrm{mmol}$ ), ethyl diazoacetate (2a, 3.0 equiv), silver 3,5-dinitrobenzoate (5 mol\%), and THF $(4.0 \mathrm{~mL})$ under $\mathrm{N}_{2}$ atmospheric conditions. The reaction mixture stirred at $35{ }^{\circ} \mathrm{C}$ for $12 \mathrm{~h}$. After cooling the reaction mixture, it was filtered through a pad of celite and then washed with DCM $(10 \mathrm{~mL}$ $x 3$ ). The combined organic layer was concentrated under reduced pressure. The organic residue was purified by chromatography on silica gel deactivated with $5 \% \mathrm{Et}_{3} \mathrm{~N}$ to give the desired product $3 \mathbf{a}^{1}-\mathbf{D}$.

\section{B. The Reaction of Quinolinium Zwitterion (1a) and D-labeled Ethyl Diazoacetate (2a-D)}

To a flame dried test tube with a triangular-shaped stir bar were added quinolinium zwitterion (1a, 0.2 mmol), D-labeled ethyl diazoacetate (2a-D, 3.0 equiv), silver 3,5-dinitrobenzoate ( $5 \mathrm{~mol} \%$ ), and THF $(4.0 \mathrm{~mL})$ under $\mathrm{N}_{2}$ atmospheric conditions. The reaction mixture stirred at $35^{\circ} \mathrm{C}$ for $12 \mathrm{~h}$. After cooling the reaction mixture, it was filtered through a pad of celite and then washed with $\mathrm{DCM}(10 \mathrm{~mL} \times 3)$. The combined organic layer was concentrated under reduced pressure. The organic residue was purified by chromatography on silica gel deactivated with $5 \% \mathrm{Et}_{3} \mathrm{~N}$ to give the desired product $3 \mathbf{a}$.

\section{The Reaction of Quinolinium Zwitterion (1a) and Ethyl Diazoacetate (2a) in the $\mathrm{D}_{2} \mathrm{O}$}

To a flame dried test tube with a triangular-shaped stir bar were added quinolinium zwitterion (1a, 0.2 $\mathrm{mmol}$ ), ethyl diazoacetate (2a, 3.0 equiv), silver 3,5-dinitrobenzoate (5 mol\%), THF (4.0 mL), and $\mathrm{D}_{2} \mathrm{O}$ $(0.1 \mathrm{~mL})$ under $\mathrm{N}_{2}$ atmospheric conditions. The reaction mixture stirred at $35^{\circ} \mathrm{C}$ for $12 \mathrm{~h}$. After cooling 
the reaction mixture, it was filtered through a pad of celite and then washed with $\mathrm{DCM}(10 \mathrm{~mL} \times 3)$. The combined organic layer was concentrated under reduced pressure. The organic residue was purified by chromatography on silica gel deactivated with $5 \% \mathrm{Et}_{3} \mathrm{~N}$ to give the desired product mixture $\left(3 \mathbf{a} / \mathbf{3 a}^{2}\right.$ D).

\section{Deprotonation of Ethyl Diazoacetate in the Presence of Silver Catalyst}

To a flame dried test tube with a triangular-shaped stir bar were added silver 3,5-dinitrobenzoate (3.0 $\mathrm{mmol})$, $2 \mathbf{a}$ (1.0 equiv), and THF- $\mathrm{d}_{8}(4.0 \mathrm{~mL})$ under $\mathrm{N}_{2}$ atmospheric conditions. The reaction mixture stirred at $35^{\circ} \mathrm{C}$ for $6 \mathrm{~h}$. The deprotonated compound $2 \mathrm{a}$ was observed through ${ }^{1} \mathrm{H}$-NMR using $1,1,2,2,-$ tetrachloroethane as an internal standard.

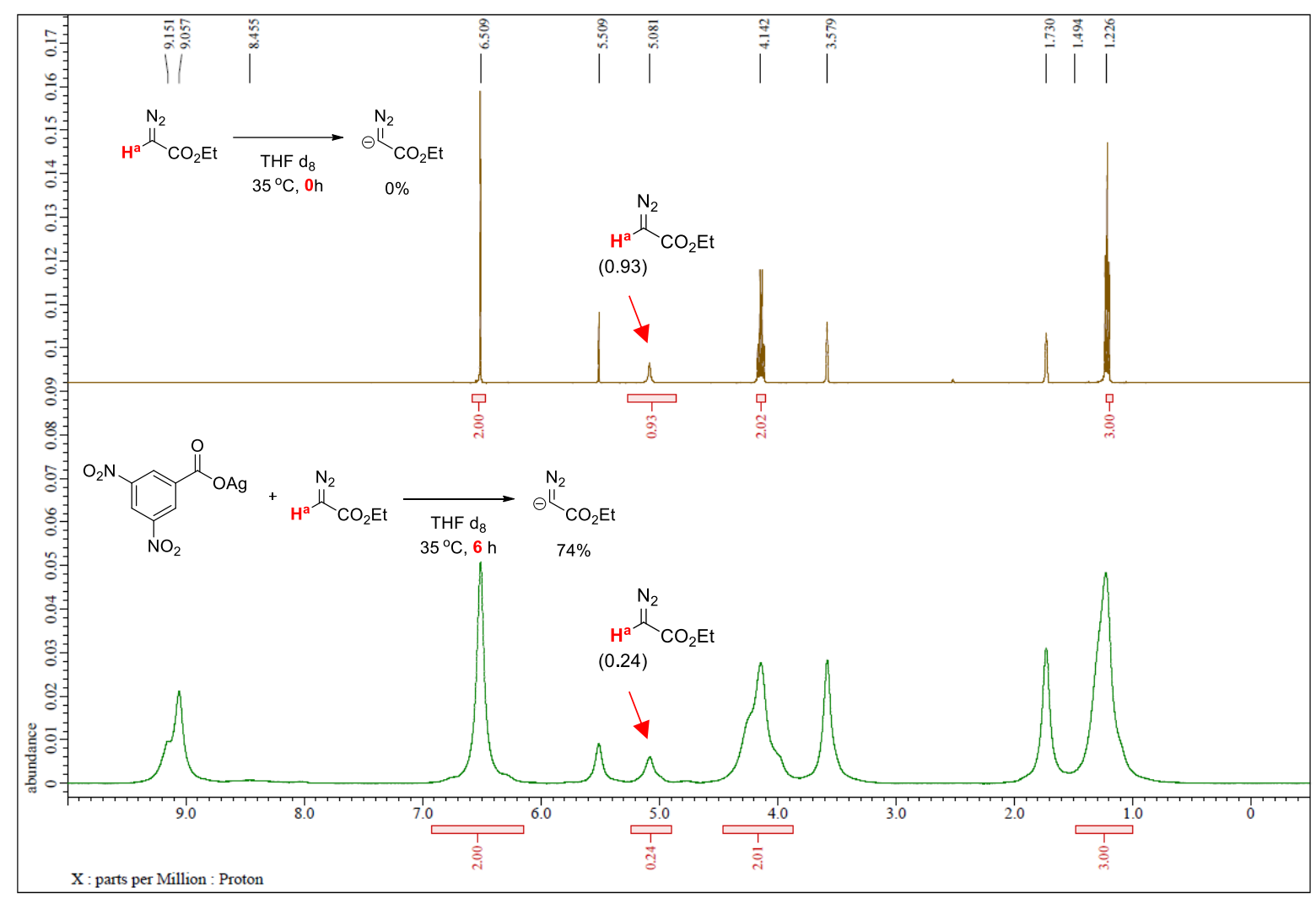

\section{E. The Reaction of Quinolinium Zwitterion (1a) and Other Diazo Compounds}

reaction of other diazo compounds

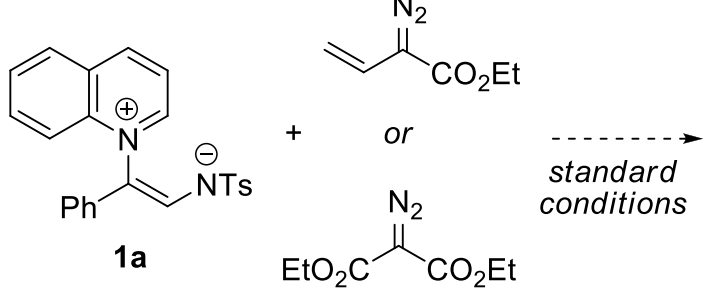

no reaction

To a flame dried test tube with a triangular-shaped stir bar were added quinolinium zwitterion $(1,0.2$ $\mathrm{mmol}$ ), diazo compound (ethyl 2-diazobut-3-enoate or diethyl 2-diazomalonate, 3.0 equiv), silver 3,5dinitrobenzoate $(5 \mathrm{~mol} \%)$, and THF $(4.0 \mathrm{~mL})$ under $\mathrm{N}_{2}$ atmospheric conditions. The reaction mixture 
stirred at $35^{\circ} \mathrm{C}$ for $12 \mathrm{~h}$. However, substrate 1a did not convert at all.

\section{Spectroscopic Data}

\section{III-1. Non-reported N-Aromatic Zwitterions}

(Z)-(2-phenyl-2-(2-deuterium-quinolin-1-ium-1-yl)vinyl)(tosyl)amide (1a-D):

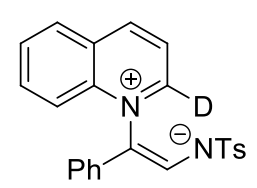

Purple Solid $(75 \%, 60.2 \mathrm{mg}) ; \mathrm{R}_{\mathrm{f}}=0.5(\mathrm{DCM} / \mathrm{MeOH}=10: 1)$; m.p. $217.4-218.8{ }^{\circ} \mathrm{C} ;{ }^{1} \mathrm{H}$ NMR (400 MHz, DMSO-d $)$ ס 9.40-9.36 (m, 1H), 8.51-8.48 (m, 1H), 8.24-8.22 (m, $0.6 \mathrm{H}), 8.08(\mathrm{~s}, 1 \mathrm{H}), 8.01-7.92(\mathrm{~m}, 3 \mathrm{H}), 7.39(\mathrm{~d}, J=8.2 \mathrm{~Hz}, 2 \mathrm{H}), 7.17(\mathrm{t}, J=7.9 \mathrm{~Hz}$, $4 \mathrm{H}), 6.99(\mathrm{t}, J=7.3 \mathrm{~Hz}, 1 \mathrm{H}), 6.79(\mathrm{~d}, J=7.5 \mathrm{~Hz}, 2 \mathrm{H}), 2.30(\mathrm{~s}, 3 \mathrm{H}) ;{ }^{13} \mathrm{C}$ NMR $(100$ $\mathrm{MHz}$, DMSO-d 6 ) $\delta 147.5,147.4,143.7,139.8,139.3,139.2,136.6,135.6,130.2$, 130.0, 129.8, 128.9, 128.6, 125.2, 123.9, 122.7, 119.7, 119.7, 113.1, 20.7; IR (liquid) v 3057, 1593, 1262, 1236, 1130, $1083 \mathrm{~cm}^{-1}$; HRMS (ESI) m/z calcd. for $\mathrm{C}_{24} \mathrm{H}_{20} \mathrm{DN}_{2} \mathrm{O}_{2} \mathrm{~S}[\mathrm{M}+\mathrm{H}]^{+}:$402.1381, found: 402.1386 .

\section{III-2. Products of the Silver-Catalyzed Ring-Expansion Reaction}

A. Azepine Derivatives (3)

ethyl 1-phenyl-3-tosyl-3a,4-dihydro-3H-benzo[f]imidazo[1,2-a]azepine-5-carboxylate (3a):<smiles>CCOC(=O)C1=Cc2ccccc2N2C(c3ccccc3)=C[NH+](S)C2C1</smiles>

Yellow Solid $(83 \%, 80.9 \mathrm{mg}) ; \mathrm{R}_{\mathrm{f}}=0.4$ (hexane/EtOAc $=4: 1$ ); m.p. $141.5-142.3{ }^{\circ} \mathrm{C}$; ${ }^{1} \mathrm{H}$ NMR $\left(400 \mathrm{MHz}, \mathrm{CDCl}_{3}\right) \delta 7.80(\mathrm{~d}, J=1.4 \mathrm{~Hz}, 1 \mathrm{H}), 7.58(\mathrm{~d}, J=8.2 \mathrm{~Hz}, 2 \mathrm{H}), 7.35$ (d, $J=7.1 \mathrm{~Hz}, 1 \mathrm{H}), 7.24-7.20(\mathrm{~m}, 3 \mathrm{H}), 7.12-7.11(\mathrm{~m}, 4 \mathrm{H}), 6.90-6.86(\mathrm{~m}, 1 \mathrm{H}), 6.73-$ $6.69(\mathrm{~m}, 1 \mathrm{H}), 6.19(\mathrm{~s}, 1 \mathrm{H}), 5.39(\mathrm{~d}, J=8.0 \mathrm{~Hz}, 1 \mathrm{H}), 4.80(\mathrm{dd}, J=9.8,2.3 \mathrm{~Hz}, 1 \mathrm{H})$, 4.31 (q, $J=7.1 \mathrm{~Hz}, 2 \mathrm{H}), 3.22-3.07(\mathrm{~m}, 2 \mathrm{H}), 2.30(\mathrm{~s}, 3 \mathrm{H}), 1.37(\mathrm{t}, J=7.2 \mathrm{~Hz}, 3 \mathrm{H}) ;{ }^{13} \mathrm{C}$ $\operatorname{NMR}\left(100 \mathrm{MHz}, \mathrm{CDCl}_{3}\right) \delta 167.8,144.3,140.7,137.7,136.5,134.7,130.7,129.1$, 128.9, 128.7, 128.6, 128.4, 128.4, 127.8, 126.7, 125.9, 122.5, 120.3, 109.1, 78.8, 61.3, 39.5, 21.4, 14.3; IR (solid) v 3128, 2923, 1705, 1164, 1155, $678 \mathrm{~cm}^{-1}$; HRMS (ESI) $\mathrm{m} / \mathrm{z}$ calcd. for $\mathrm{C}_{28} \mathrm{H}_{26} \mathrm{~N}_{2} \mathrm{NaO}_{4} \mathrm{~S}$ [M+Na]+: 509.1505, found: 509.1508 .

\section{ethyl 7-bromo-1-phenyl-3-tosyl-3a,4-dihydro-3H-benzo[f]imidazo[1,2-a]azepine-5-carboxylate}

(3b):<smiles>CCOC(=O)C1=Cc2c(Br)cccc2N2CC1C=[N+](S)C=C2c1ccccc1</smiles>

Yellow Solid $\left(52 \%, 58.8 \mathrm{mg}\right.$ ); $\mathrm{R}_{\mathrm{f}}=0.4$ (hexane/EtOAc $\left.=4: 1\right)$; m.p. $148.8-149.9{ }^{\circ} \mathrm{C}$; ${ }^{1} \mathrm{H}$ NMR $\left(400 \mathrm{MHz}, \mathrm{CDCl}_{3}\right) \delta 8.30(\mathrm{~s}, 1 \mathrm{H}), 7.58(\mathrm{~d}, J=8.2 \mathrm{~Hz}, 2 \mathrm{H}), 7.28-7.09(\mathrm{~m}$, $8 \mathrm{H}), 6.53(\mathrm{t}, J=8.0 \mathrm{~Hz}, 1 \mathrm{H}), 6.24(\mathrm{~s}, 1 \mathrm{H}), 5.33(\mathrm{~d}, J=8.0 \mathrm{~Hz}, 1 \mathrm{H}), 4.81(\mathrm{t}, J=6.6$ $\mathrm{Hz}, 1 \mathrm{H}), 4.35-4.30(\mathrm{~m}, 2 \mathrm{H}), 3.16-3.14(\mathrm{~m}, 2 \mathrm{H}), 2.31(\mathrm{~s}, 3 \mathrm{H}), 1.38(\mathrm{t}, \mathrm{J}=7.1 \mathrm{~Hz}, 3 \mathrm{H})$; ${ }^{13} \mathrm{C} \mathrm{NMR}\left(100 \mathrm{MHz}, \mathrm{CDCl}_{3}\right) \delta$ 167.5, 144.3, 143.1, 136.1, 135.7, 130.7, 130.6, 129.2, $129.0,128.7,128.5,128.2,127.9,127.6,126.5,125.5,119.7,110.0,79.4,61.4$, 39.0, 21.4, 14.3; IR (solid) v 3122, 2925, 1700, 1244, 1166, $668 \mathrm{~cm}^{-1}$; HRMS (ESI) $\mathrm{m} / \mathrm{z}$ calcd. for $\mathrm{C}_{28} \mathrm{H}_{25} \mathrm{BrN}_{2} \mathrm{NaO}_{4} \mathrm{~S}[\mathrm{M}+\mathrm{Na}]^{+}: 587.0611$, found: 587.0613 .

ethyl 8-methoxy-1-phenyl-3-tosyl-3a,4-dihydro-3H-benzo[f]imidazo[1,2-a]azepine-5-carboxylate (3c):<smiles>CCOC(=O)C1=Cc2cc(OC)ccc2N2C(c3ccccc3)=C[N+]([S+])C2C1</smiles>
Yellow Solid $(62 \%, 63.7 \mathrm{mg}) ; \mathrm{R}_{\mathrm{f}}=0.4$ (hexane/EtOAc $=4: 1$ ); m.p. 126.6$127.3^{\circ} \mathrm{C} ;{ }^{1} \mathrm{H}$ NMR $\left(400 \mathrm{MHz}, \mathrm{CDCl}_{3}\right) \delta 7.79(\mathrm{~d}, J=1.6 \mathrm{~Hz}, 1 \mathrm{H}), 7.57(\mathrm{~d}, J=$ $8.2 \mathrm{~Hz}, 2 \mathrm{H}), 7.34(\mathrm{~d}, J=6.9 \mathrm{~Hz}, 1 \mathrm{H}), 7.13(\mathrm{t}, J=8.1 \mathrm{~Hz}, 3 \mathrm{H}), 6.88(\mathrm{td}, J=7.5$, $0.9 \mathrm{~Hz}, 1 \mathrm{H}), 6.79(\mathrm{dd}, J=8.2,1.8 \mathrm{~Hz}, 1 \mathrm{H}), 6.74-6.68(\mathrm{~m}, 2 \mathrm{H}), 6.63(\mathrm{t}, J=1.8$ $\mathrm{Hz}, 1 \mathrm{H}), 6.19(\mathrm{~s}, 1 \mathrm{H}), 5.42(\mathrm{~d}, J=7.8 \mathrm{~Hz}, 1 \mathrm{H}), 4.80-4.77(\mathrm{~m}, 1 \mathrm{H}), 4.34-4.26$ $(\mathrm{m}, 2 \mathrm{H}), 3.66(\mathrm{~s}, 3 \mathrm{H}), 3.21-3.06(\mathrm{~m}, 2 \mathrm{H}), 2.30(\mathrm{~s}, 3 \mathrm{H}), 1.36(\mathrm{t}, \mathrm{J}=7.1 \mathrm{~Hz}, 3 \mathrm{H})$; ${ }^{13} \mathrm{C}$ NMR $\left(100 \mathrm{MHz}, \mathrm{CDCl}_{3}\right) \delta 167.8,159.5,144.3,140.7,137.7,136.4,134.7,130.6,130.1,129.7$, 129.1, 128.5, 128.3, 127.8, 125.8, 122.5, 120.2, 119.2, 114.2, 112.3, 109.3, 78.8, 61.3, 55.2, 39.5, 21.4 , 14.3; IR (solid) v 3125, 1704, 1357, 1251, 1220, $1170 \mathrm{~cm}^{-1}$; HRMS (ESI) m/z calcd. for $\mathrm{C}_{29} \mathrm{H}_{28} \mathrm{~N}_{2} \mathrm{NaO}_{5} \mathrm{~S}$ $[\mathrm{M}+\mathrm{Na}]^{+}: 539.1611$, found: 539.1614 . 
(3d):<smiles>CCOC(=O)C1=Cc2cc(F)ccc2N2C(c3ccccc3)=C[NH+](S)C2C1</smiles>

Yellow Solid $(67 \%, 67.6 \mathrm{mg}) ; \mathrm{R}_{\mathrm{f}}=0.4$ (hexane/EtOAc $\left.=4: 1\right) ; \mathrm{m} . \mathrm{p} \cdot 147.8-148.4^{\circ} \mathrm{C}$; ${ }^{1} \mathrm{H}$ NMR $\left(300 \mathrm{MHz}, \mathrm{CDCl}_{3}\right) \delta 7.70(\mathrm{~s}, 1 \mathrm{H}), 7.59(\mathrm{~d}, J=8.1 \mathrm{~Hz}, 2 \mathrm{H}), 7.25-7.04(\mathrm{~m}$, $8 \mathrm{H}), 6.47-6.40(\mathrm{~m}, 1 \mathrm{H}), 6.19(\mathrm{~s}, 1 \mathrm{H}), 5.33(\mathrm{dd}, J=8.9,5.0 \mathrm{~Hz}, 1 \mathrm{H}), 4.76(\mathrm{dd}, J=$ 9.2, $2.9 \mathrm{~Hz}, 1 \mathrm{H}), 4.31(\mathrm{q}, J=7.1 \mathrm{~Hz}, 2 \mathrm{H}), 3.24-3.06(\mathrm{~m}, 2 \mathrm{H}), 2.32(\mathrm{~s}, 3 \mathrm{H}), 1.37(\mathrm{t}$, $J=7.2 \mathrm{~Hz}, 3 \mathrm{H}) ;{ }^{13} \mathrm{C}$ NMR $\left(75 \mathrm{MHz}, \mathrm{CDCl}_{3}\right) \delta 167.4,157.9(\mathrm{~d}, J=241.5 \mathrm{~Hz})$, $144.3,137.0,136.3,136.2,130.5,130.1,129.1,129.0,128.7,128.4,127.8$, $127.3(\mathrm{~d}, J=7.6 \mathrm{~Hz}), 126.6,121.3(\mathrm{~d}, J=7.6 \mathrm{~Hz}), 119.9(\mathrm{~d}, J=23.0 \mathrm{~Hz}), 115.4(\mathrm{~d}, J=23.0 \mathrm{~Hz}), 108.9$, 78.7, 61.4, 39.6, 21.4, 14.3; IR (solid) v 1686, 1494, 1351, 1221, 1158, $677 \mathrm{~cm}^{-1}$; HRMS (ESI) m/z calcd. for $\mathrm{C}_{28} \mathrm{H}_{26} \mathrm{FN}_{2} \mathrm{O}_{4} \mathrm{~S}[\mathrm{M}+\mathrm{H}]^{+}:$505.1592, found: 505.1594 .

ethyl 10-methoxy-1-phenyl-4H-benzo[f]imidazo[1,2-a]azepine-5-carboxylate (3e'):

White Solid $\left(46 \%, 33.2 \mathrm{mg}\right.$ ); $\mathrm{R}_{\mathrm{f}}=0.4$ (hexane/EtOAc $=2: 1$ ); m.p.88.1-90.7 ${ }^{\circ} \mathrm{C} ;{ }^{1} \mathrm{H}$<smiles>CCOC(=O)C1=Cc2cccc(OC)c2-n2c(-c3ccccc3)cnc2C1</smiles>
$\operatorname{NMR}\left(400 \mathrm{MHz}, \mathrm{CDCl}_{3}\right) \delta 7.79(\mathrm{~s}, 1 \mathrm{H}), 7.33(\mathrm{t}, J=8.0 \mathrm{~Hz}, 1 \mathrm{H}), 7.23-7.12(\mathrm{~m}, 4 \mathrm{H})$, $7.08(\mathrm{~s}, 1 \mathrm{H}), 6.98-6.96(\mathrm{~m}, 2 \mathrm{H}), 6.75(\mathrm{~d}, J=8.2 \mathrm{~Hz}, 1 \mathrm{H}), 4.43-4.26(\mathrm{~m}, 3 \mathrm{H}), 3.06-$ $3.02(\mathrm{~m}, 4 \mathrm{H}), 1.38(\mathrm{t}, J=7.1 \mathrm{~Hz}, 3 \mathrm{H}) ;{ }^{13} \mathrm{C}$ NMR $\left(100 \mathrm{MHz}, \mathrm{CDCl}_{3}\right) \delta 165.3,152.4$, 151.7, 136.3, 135.6, 133.9, 132.0, 131.7, 128.1, 127.8, 127.2, 126.8, 125.2, 124.7, 122.3, 112.4, 61.6, 54.7, 26.4, 14.2; IR (liquid) v 3049, 2978, 1705, 1473, 1272 , 1214, $736 \mathrm{~cm}^{-1}$; HRMS (ESI) m/z calcd. for $\mathrm{C}_{22} \mathrm{H}_{21} \mathrm{~N}_{2} \mathrm{O}_{3}[\mathrm{M}+\mathrm{H}]^{+}: 361.1547$, found: 361.1550 .

ethyl 6-phenyl-4-tosyl-3a,4-dihydro-3H-imidazo[1',2':1,7]azepino[2,3-h]quinoline-2-carboxylate (3f):<smiles>CCOC(=O)C1=Cc2c(ccc3cccnc23)N2C(c3ccccc3)=C[NH+]C2C1</smiles>

Yellow Solid $\left(66 \%, 68.7 \mathrm{mg}\right.$ ); $\mathrm{R}_{\mathrm{f}}=0.4$ (hexane/EtOAc $=2: 1$ ); m.p.159.0-159.7 ${ }^{\circ} \mathrm{C}$; ${ }^{1} \mathrm{H}$ NMR $\left(300 \mathrm{MHz}, \mathrm{CDCl}_{3}\right) \delta 9.45(\mathrm{~d}, J=1.7 \mathrm{~Hz}, 1 \mathrm{H}), 8.96(\mathrm{dd}, J=4.3,1.7 \mathrm{~Hz}$, $1 \mathrm{H}), 7.93(\mathrm{dd}, J=8.1,1.7 \mathrm{~Hz}, 1 \mathrm{H}), 7.57(\mathrm{~d}, J=8.3 \mathrm{~Hz}, 2 \mathrm{H}), 7.34(\mathrm{q}, J=4.1 \mathrm{~Hz}$, $1 \mathrm{H}), 7.27-7.12(\mathrm{~m}, 6 \mathrm{H}), 7.05(\mathrm{~d}, J=8.1 \mathrm{~Hz}, 2 \mathrm{H}), 6.30(\mathrm{~s}, 1 \mathrm{H}), 5.72(\mathrm{~d}, J=8.8 \mathrm{~Hz}$, $1 \mathrm{H}), 4.92(\mathrm{dd}, J=9.7,2.9 \mathrm{~Hz}, 1 \mathrm{H}), 4.44-4.28(\mathrm{~m}, 2 \mathrm{H}), 3.38-3.17(\mathrm{~m}, 2 \mathrm{H}), 2.29$ (s, $3 \mathrm{H}), 1.40(\mathrm{t}, J=7.1 \mathrm{~Hz}, 3 \mathrm{H}) ;{ }^{13} \mathrm{C}$ NMR $\left(100 \mathrm{MHz}, \mathrm{CDCl}_{3}\right) \delta 168.1,150.3,147.7$, 144.2, 141.9, 136.2, 135.9, 131.9, 131.1, 129.2, 129.2, 128.8, 127.9, 127.8, 126.6, 124.2, 121.0, 120.5, 120.0, 110.1, 79.6, 61.2, 39.5, 21.4, 14.4; IR (solid) v 2955, 1698, 1596, 1348, 1249, $1165 \mathrm{~cm}^{-1}$; HRMS (ESI) $\mathrm{m} / \mathrm{z}$ calcd. for $\mathrm{C}_{31} \mathrm{H}_{28} \mathrm{~N}_{3} \mathrm{O}_{4} \mathrm{~S}[\mathrm{M}+\mathrm{H}]^{+}:$538.1795, found: 538.1802.

ethyl carboxylate $(3 \mathrm{~g})$ :<smiles>CCOC(=O)C1=Cc2ccccc2N2C(c3ccc(C(C)(C)C)cc3)=C[NH+](S)C2C1</smiles>
1-(4-(tert-butyl)phenyl)-3-tosyl-3a,4-dihydro-3H-benzo[f]imidazo[1,2-a]azepine-5130.6, 129.1, 128.5, 128.4, 127.8, 126.3, 125.8, 125.7, 125.5, 122.4, 120.4, 108.6, 78.8, 61.2, 39.4, 34.7, 31.1, 21.4, 14.3; IR (solid) v 3120, 2955, 1709, 1240, 1198, 765, $670 \mathrm{~cm}^{-1}$; HRMS (ESI) m/z calcd. for $\mathrm{C}_{32} \mathrm{H}_{34} \mathrm{~N}_{2} \mathrm{NaO}_{4} \mathrm{~S}$ [M+Na]+: 565.2131, found: 565.2132 .

ethyl 3-tosyl-1-(4-(trifluoromethyl)phenyl)-3a,4-dihydro-3H-benzo[f]imidazo[1,2-a]azepine-5carboxylate (3h): 
<smiles>CCOC(=O)C1=Cc2ccccc2N2C(c3ccc(C(F)(F)F)cc3)=C[NH+]([SH+](=O)S)CC2C1</smiles>

Yellow Solid $\left(73 \%, 80.7 \mathrm{mg}\right.$ ); $\mathrm{R}_{\mathrm{f}}=0.4$ (hexane/EtOAc $=4: 1$ ); m.p.160.3$161.1^{\circ} \mathrm{C} ;{ }^{1} \mathrm{H}$ NMR $\left(400 \mathrm{MHz}, \mathrm{CDCl}_{3}\right) \delta 7.80(\mathrm{~s}, 1 \mathrm{H}), 7.58(\mathrm{~d}, J=8.0 \mathrm{~Hz}, 2 \mathrm{H})$, $7.47(\mathrm{~d}, J=8.2 \mathrm{~Hz}, 2 \mathrm{H}), 7.37(\mathrm{~d}, J=7.5 \mathrm{~Hz}, 1 \mathrm{H}), 7.22(\mathrm{~d}, J=8.2 \mathrm{~Hz}, 2 \mathrm{H}), 7.13$ (d, $J=8.2 \mathrm{~Hz}, 2 \mathrm{H}), 6.92$ (t, $J=7.5 \mathrm{~Hz}, 1 \mathrm{H}), 6.75(\mathrm{t}, J=7.4 \mathrm{~Hz}, 1 \mathrm{H}), 6.31$ (s, $1 \mathrm{H}), 5.38(\mathrm{~d}, J=8.0 \mathrm{~Hz}, 1 \mathrm{H}), 4.84(\mathrm{dd}, J=10.1,2.5 \mathrm{~Hz}, 1 \mathrm{H}), 4.31(\mathrm{q}, J=7.1$ $\mathrm{Hz}, 2 \mathrm{H}), 3.24-3.07(\mathrm{~m}, 2 \mathrm{H}), 2.31(\mathrm{~s}, 3 \mathrm{H}), 1.37(\mathrm{t}, J=7.2 \mathrm{~Hz}, 3 \mathrm{H}) ;{ }^{13} \mathrm{C}$ NMR $\left(100 \mathrm{MHz}, \mathrm{CDCl}_{3}\right) \delta 167.7,144.5,140.5,137.6,134.9,134.7,132.5,130.9$, $130.7,130.4,129.3,128.7,128.6,127.8,126.7,126.1,125.6,125.6,125.1,123.0,122.4,120.0,111.2$, 79.2, 61.3, 39.7, 21.4, 14.3; IR (solid) v 3065, 1710, 1324, 1155, 1124, $851 \mathrm{~cm}^{-1} ; \mathrm{HRMS}$ (ESI) m/z calcd. for $\mathrm{C}_{29} \mathrm{H}_{25} \mathrm{~F}_{3} \mathrm{~N}_{2} \mathrm{NaO}_{4} \mathrm{~S}[\mathrm{M}+\mathrm{Na}]^{+}: 577.1379$, found: 577.1384 .

ethyl 1-(3-methoxyphenyl)-3-tosyl-3a,4-dihydro-3H-benzo[f]imidazo[1,2-a]azepine-5-carboxylate (3i):

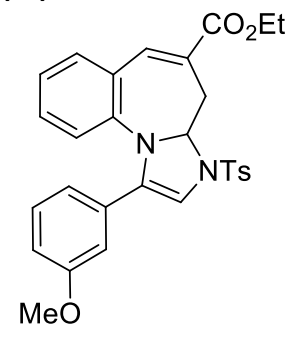

Yellow Solid $(63 \%, 62.9 \mathrm{mg}) ; \mathrm{R}_{\mathrm{f}}=0.4$ (hexane/EtOAc $\left.=4: 1\right) ;$ m.p.119.2-120.3 ${ }^{\circ} \mathrm{C}$; ${ }^{1} \mathrm{H}$ NMR $\left(400 \mathrm{MHz}, \mathrm{CDCl}_{3}\right) \delta 7.79(\mathrm{~d}, J=1.6 \mathrm{~Hz}, 1 \mathrm{H}), 7.58(\mathrm{~d}, J=8.2 \mathrm{~Hz}, 2 \mathrm{H})$, 7.35-7.33 (m, $1 \mathrm{H}), 7.13(\mathrm{t}, J=7.9 \mathrm{~Hz}, 3 \mathrm{H}), 6.88(\mathrm{td}, J=7.5,1.1 \mathrm{~Hz}, 1 \mathrm{H}), 6.80-6.77$ $(\mathrm{m}, 1 \mathrm{H}), 6.74-6.68(\mathrm{~m}, 2 \mathrm{H}), 6.63(\mathrm{t}, J=2.1 \mathrm{~Hz}, 1 \mathrm{H}), 6.19(\mathrm{~d}, J=0.7 \mathrm{~Hz}, 1 \mathrm{H}), 5.44$ $(\mathrm{d}, J=8.0 \mathrm{~Hz}, 1 \mathrm{H}), 4.81-4.78(\mathrm{~m}, 1 \mathrm{H}), 4.30(\mathrm{q}, J=7.1 \mathrm{~Hz}, 2 \mathrm{H}), 3.66(\mathrm{~s}, 3 \mathrm{H}), 3.22-$ $3.06(\mathrm{~m}, 2 \mathrm{H}), 2.30(\mathrm{~s}, 3 \mathrm{H}), 1.37(\mathrm{t}, J=3.5 \mathrm{~Hz}, 3 \mathrm{H}) ;{ }^{13} \mathrm{C} \mathrm{NMR}\left(100 \mathrm{MHz}, \mathrm{CDCl}_{3}\right) \delta$ $167.8,159.6,144.3,140.7,137.7,136.3,134.7,130.8,130.1,129.7,129.2,128.5$, $128.4,127.8,125.9,122.5,120.3,119.2,114.2,112.4,109.4,78.9,61.3,55.2$, 39.5, 21.4, 14.3; IR (solid) v 2962, 1703, 1351, 1235, 1195, $1161 \mathrm{~cm}^{-1}$; HRMS (ESI) m/z calcd. for $\mathrm{C}_{29} \mathrm{H}_{28} \mathrm{~N}_{2} \mathrm{NaO}_{5} \mathrm{~S}[\mathrm{M}+\mathrm{Na}]^{+}: 539.1611$, found: 539.1615 .

\section{ethyl 1-(3-fluorophenyl)-3-tosyl-3a,4-dihydro-3H-benzo[f]imidazo[1,2-a]azepine-5-carboxylate}

(3j):

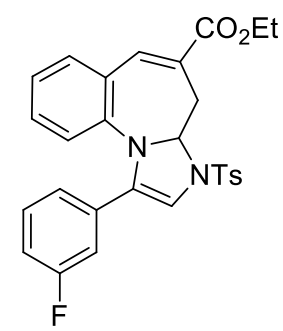

Yellow Solid $(83 \%, 83.9 \mathrm{mg}) ; R_{\mathrm{f}}=0.4$ (hexane/EtOAc $=4: 1$ ); m.p. $148.3-148.8^{\circ} \mathrm{C}$; ${ }^{1} \mathrm{H} \mathrm{NMR}\left(400 \mathrm{MHz}, \mathrm{CDCl}_{3}\right) \delta 7.80(\mathrm{~d}, J=1.1 \mathrm{~Hz}, 1 \mathrm{H}), 7.58(\mathrm{~d}, J=8.2 \mathrm{~Hz}, 2 \mathrm{H}), 7.36$ $(\mathrm{d}, J=7.5 \mathrm{~Hz}, 1 \mathrm{H}), 7.21-7.12(\mathrm{~m}, 3 \mathrm{H}), 6.96-6.90(\mathrm{~m}, 3 \mathrm{H}), 6.82-6.73(\mathrm{~m}, 2 \mathrm{H}), 6.24$ (s, 1H), $5.42(\mathrm{~d}, J=8.2 \mathrm{~Hz}, 1 \mathrm{H}), 4.82(\mathrm{dd}, J=9.9,2.4 \mathrm{~Hz}, 1 \mathrm{H}), 4.31(\mathrm{q}, J=7.1 \mathrm{~Hz}$, 2H), 3.23-3.07 (m, 2H), 2.31 (s, 3H), 1.37 (t, $J=7.1 \mathrm{~Hz}, 3 \mathrm{H}) ;{ }^{13} \mathrm{C} \mathrm{NMR}(100 \mathrm{MHz}$, $\left.\mathrm{CDCl}_{3}\right) \delta 167.7,162.6(\mathrm{~d}, J=245.3 \mathrm{~Hz}), 144.4,140.6,137.6,135.1,134.8,131.0$ $(\mathrm{d}, J=8.6 \mathrm{~Hz}), 130.7,130.3(\mathrm{~d}, J=7.6 \mathrm{~Hz}), 129.2,128.6,128.4,127.8,126.0$, $122.8,122.3(\mathrm{~d}, J=2.9 \mathrm{~Hz}), 120.0,115.8(\mathrm{~d}, J=21.1 \mathrm{~Hz}), 113.4(\mathrm{~d}, J=23.0 \mathrm{~Hz})$, 110.3, 79.1, 61.2, 39.6, 21.4, 14.3; IR (solid) v 3116, 1703, 1353, 1258, 1193, 1155, $674 \mathrm{~cm}^{-1}$; HRMS (ESI) $\mathrm{m} / \mathrm{z}$ calcd. for $\mathrm{C}_{28} \mathrm{H}_{25} \mathrm{FN}_{2} \mathrm{NaO}_{4} \mathrm{~S}[\mathrm{M}+\mathrm{Na}]^{+}: 527.1411$, found: 527.1415 .

\section{ethyl 1-(2-fluorophenyl)-3-tosyl-3a,4-dihydro-3H-benzo[f]imidazo[1,2-a]azepine-5-carboxylate}

(3k):<smiles>CCOC(=O)C1=Cc2ccccc2N2C(c3ccccc3F)=C[NH+]([SH+])C2C1</smiles>

Yellow Solid $(80 \%, 80.3 \mathrm{mg}) ; R_{\mathrm{f}}=0.4$ (hexane/EtOAc $\left.=4: 1\right) ;$ m.p. $149.2-149.9{ }^{\circ} \mathrm{C}$, ${ }^{1} \mathrm{H}$ NMR $\left(400 \mathrm{MHz}, \mathrm{CDCl}_{3}\right) \delta 7.79(\mathrm{~s}, 1 \mathrm{H}), 7.60(\mathrm{~d}, J=8.2 \mathrm{~Hz}, 2 \mathrm{H}), 7.35-7.31(\mathrm{~m}$, $1 \mathrm{H}), 7.22-7.17(\mathrm{~m}, 1 \mathrm{H}), 7.13-7.05(\mathrm{~m}, 3 \mathrm{H}), 6.88(\mathrm{td}, J=7.4,3.0 \mathrm{~Hz}, 2 \mathrm{H}), 6.81-6.78$ $(\mathrm{m}, 1 \mathrm{H}), 6.72(\mathrm{t}, J=7.3 \mathrm{~Hz}, 1 \mathrm{H}), 6.45(\mathrm{~d}, J=1.6 \mathrm{~Hz}, 1 \mathrm{H}), 5.41(\mathrm{~d}, J=8.0 \mathrm{~Hz}, 1 \mathrm{H})$, $4.75(\mathrm{dd}, J=9.9,2.6 \mathrm{~Hz}, 1 \mathrm{H}), 4.30(\mathrm{q}, J=7.1 \mathrm{~Hz}, 2 \mathrm{H}), 3.22-3.07(\mathrm{~m}, 2 \mathrm{H}), 2.30(\mathrm{~s}$, $3 \mathrm{H}), 1.36(\mathrm{t}, J=7.1 \mathrm{~Hz}, 3 \mathrm{H}) ;{ }^{13} \mathrm{C}$ NMR $\left(100 \mathrm{MHz}, \mathrm{CDCl}_{3}\right) \delta 167.8,159.9(\mathrm{~d}, \mathrm{~J}=$ $250.1 \mathrm{~Hz}), 144.3,141.0,137.6,134.7,130.4,129.8(\mathrm{~d}, J=8.6 \mathrm{~Hz}), 129.2,129.1$, 128.8, 128.6, 128.5, 127.9, 125.9, 124.0 (d, $J=2.9 \mathrm{~Hz}), 122.6,119.5,116.8$ (d, $J=11.5 \mathrm{~Hz}), 116.1$ (d, $J=22.1 \mathrm{~Hz}$ ), $113.2(\mathrm{~d}, J=8.7 \mathrm{~Hz}), 78.2,61.3,39.6,21.4,14.3$; IR (solid) v 3122, 1703, 1492, 1353, $1243,1156,756 \mathrm{~cm}^{-1}$; HRMS (ESI) $\mathrm{m} / \mathrm{z}$ calcd. for $\mathrm{C}_{28} \mathrm{H}_{25} \mathrm{FN}_{2} \mathrm{NaO}_{4} \mathrm{~S}[\mathrm{M}+\mathrm{Na}]^{+}: 527.1411$, found: 527.1415 .

\section{ethyl 1-(3,5-difluorophenyl)-3-tosyl-3a,4-dihydro-3H-benzo[f]imidazo[1,2-a]azepine} -5-carboxylate (3I): 


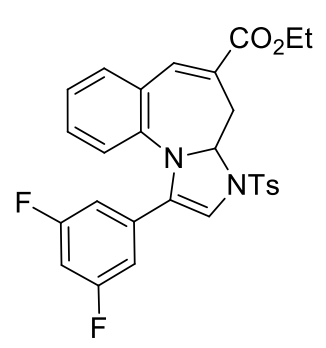

Yellow Solid $\left(70 \%, 73.0 \mathrm{mg}\right.$ ); $\mathrm{R}_{\mathrm{f}}=0.4$ (hexane/EtOAc $=4: 1$ ); m.p. 162.0$163.0{ }^{\circ} \mathrm{C} ;{ }^{1} \mathrm{H} \mathrm{NMR}\left(400 \mathrm{MHz}, \mathrm{CDCl}_{3}\right) \delta 7.79(\mathrm{~d}, J=1.8 \mathrm{~Hz}, 1 \mathrm{H}), 7.56(\mathrm{~d}, J=8.2$ $\mathrm{Hz}, 2 \mathrm{H}), 7.37(\mathrm{~d}, J=7.1 \mathrm{~Hz}, 1 \mathrm{H}), 7.15(\mathrm{~d}, J=8.0 \mathrm{~Hz}, 2 \mathrm{H}), 6.95-6.91(\mathrm{~m}, 1 \mathrm{H})$, 6.80-6.76 (m, 1H), 6.71-6.60 (m, 3H), $6.27(\mathrm{~s}, 1 \mathrm{H}), 5.44(\mathrm{~d}, J=8.0 \mathrm{~Hz}, 1 \mathrm{H}), 4.82$ (dd, $J=9.8,2.5 \mathrm{~Hz}, 1 \mathrm{H}), 4.31(\mathrm{q}, J=7.2 \mathrm{~Hz}, 2 \mathrm{H}), 3.22-3.05(\mathrm{~m}, 2 \mathrm{H}), 2.32$ (s, $3 \mathrm{H}), 1.37(\mathrm{t}, J=7.1 \mathrm{~Hz}, 3 \mathrm{H}) ;{ }^{13} \mathrm{C} \mathrm{NMR}\left(100 \mathrm{MHz}, \mathrm{CDCl}_{3}\right) \delta 167.7,163.0(\mathrm{~d}, J=$ $248.2 \mathrm{~Hz}), 162.9(\mathrm{~d}, J=248.1 \mathrm{~Hz}), 144.6,140.5,137.6,134.9,134.0,132.2(\mathrm{t}$, $J=9.6 \mathrm{~Hz}), 130.7,129.3,128.8,128.5,127.7,126.1,123.1,119.7,111.4,109.4$

(dd, $J=19.2,7.7 \mathrm{~Hz}$ ), 102.2 (t, $J=25.8 \mathrm{~Hz}$ ), 79.3, 61.3, 39.7, 21.5, 14.3; IR (solid) v 2988, 1701, 1589 , 1355, 1155, $666 \mathrm{~cm}^{-1}$; HRMS (ESI) $\mathrm{m} / \mathrm{z}$ calcd. for $\mathrm{C}_{28} \mathrm{H}_{24} \mathrm{~F}_{2} \mathrm{~N}_{2} \mathrm{NaO}_{4} \mathrm{~S}$ [M+Na] $]^{+}$: 545.1317, found: 545.1322.

ethyl 1-phenyl-3-(phenylsulfonyl)-3a,4-dihydro-3H-benzo[f]imidazo[1,2-a]azepine-5-carboxylate $(3 \mathrm{~m})$ :<smiles>CCOC(=O)C1=Cc2ccccc2N2C(c3ccccc3)=CN(S(=O)(=O)c3ccccc3)C2C1</smiles>

Yellow Solid $\left(67 \%, 62.9 \mathrm{mg}\right.$ ); $\mathrm{R}_{\mathrm{f}}=0.4$ (hexane/EtOAc $=4: 1$ ); m.p. 122.8$123.6{ }^{\circ} \mathrm{C} ;{ }^{1} \mathrm{H}$ NMR $\left(400 \mathrm{MHz}, \mathrm{CDCl}_{3}\right) \delta 7.81(\mathrm{~d}, J=1.8 \mathrm{~Hz}, 1 \mathrm{H}), 7.73-7.71(\mathrm{~m}$, 2H), 7.47 (t, $J=7.4 \mathrm{~Hz}, 1 \mathrm{H}), 7.36(\mathrm{t}, J=7.5 \mathrm{~Hz}, 3 \mathrm{H}), 7.25-7.19(\mathrm{~m}, 3 \mathrm{H}), 7.09$ (dd, $J=8.0,1.4 \mathrm{~Hz}, 2 \mathrm{H}$ ), $6.88(\mathrm{td}, J=7.5,1.0 \mathrm{~Hz}, 1 \mathrm{H}), 6.74-6.70(\mathrm{~m}, 1 \mathrm{H}), 6.20$ $(\mathrm{d}, J=0.7 \mathrm{~Hz}, 1 \mathrm{H}), 5.39(\mathrm{~d}, J=8.0 \mathrm{~Hz}, 1 \mathrm{H}), 4.87-4.84(\mathrm{~m}, 1 \mathrm{H}), 4.31(\mathrm{q}, J=7.1$ $\mathrm{Hz}, 2 \mathrm{H}), 3.24-3.09(\mathrm{~m}, 2 \mathrm{H}), 1.37$ (t, $J=7.1 \mathrm{~Hz}, 3 \mathrm{H}) ;{ }^{13} \mathrm{C} \mathrm{NMR}\left(100 \mathrm{MHz}, \mathrm{CDCl}_{3}\right)$ $\delta 167.8,140.5,137.7,136.6,134.7,134.0,133.3,128.9,128.8,128.6,128.6$, 128.3, 127.8, 126.7, 125.9, 122.6, 120.5, 108.7, 78.7, 61.3, 39.5, 14.3; IR (solid) v 2919, 1701, 1237, 1195, 1170, 714, $692 \mathrm{~cm}^{-1}$; HRMS (ESI) m/z calcd. for $\mathrm{C}_{27} \mathrm{H}_{24} \mathrm{~N}_{2} \mathrm{NaO}_{4} \mathrm{~S}[\mathrm{M}+\mathrm{Na}]^{+}:$495.1349, found: 495.1352.

ethyl 3-((4-methoxyphenyl)sulfonyl)-1-phenyl-3a,4-dihydro-3H-benzo[ $f$ imidazo [1,2-a]azepine-5-carboxylate (3n):

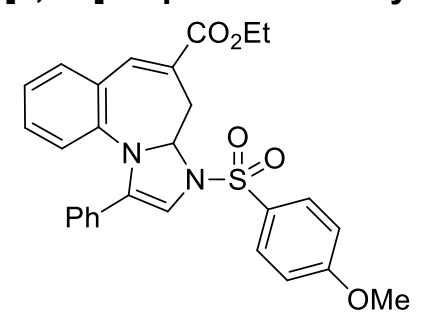

Orange Solid $\left(78 \%, 78.0 \mathrm{mg}\right.$ ); $\mathrm{R}_{\mathrm{f}}=0.4$ (hexane/EtOAc $=4: 1$ ); m.p. 124.4125.0 ${ }^{\circ} \mathrm{C} ;{ }^{1} \mathrm{H} \mathrm{NMR}\left(300 \mathrm{MHz}, \mathrm{CDCl}_{3}\right) \delta 7.81(\mathrm{~s}, 1 \mathrm{H}), 7.64(\mathrm{~d}, J=8.6 \mathrm{~Hz}$, $2 \mathrm{H}), 7.36(\mathrm{~d}, J=7.5 \mathrm{~Hz}, 1 \mathrm{H}), 7.27-7.20(\mathrm{~m}, 3 \mathrm{H}), 7.13-7.10(\mathrm{~m}, 2 \mathrm{H}), 6.89$ (t, $J=7.5 \mathrm{~Hz}, 1 \mathrm{H}), 6.79-6.70(\mathrm{~m}, 3 \mathrm{H}), 6.19(\mathrm{~s}, 1 \mathrm{H}), 5.45(\mathrm{~d}, J=7.9 \mathrm{~Hz}$, $1 \mathrm{H}), 4.81(\mathrm{dd}, J=9.4,3.1 \mathrm{~Hz}, 1 \mathrm{H}), 4.30(\mathrm{q}, J=7.1 \mathrm{~Hz}, 2 \mathrm{H}), 3.74(\mathrm{~s}, 3 \mathrm{H})$, 3.23-3.06 (m, 2H), $1.37(\mathrm{t}, J=7.1 \mathrm{~Hz}, 3 \mathrm{H}) ;{ }^{13} \mathrm{C} \mathrm{NMR}\left(75 \mathrm{MHz}, \mathrm{CDCl}_{3}\right) \delta$ $167.8,163.4,140.7,137.6,136.5,134.7,129.9,128.9,128.7,128.6$, $128.5,128.4,126.6,125.8,125.3,122.4,120.4,113.7,109.1,78.9,61.2$,

55.5, 39.5, 14.3; IR (solid) v 3045, 1702, 1492, 1243, 1198, 1155, $678 \mathrm{~cm}^{-1}$; HRMS (ESI) m/z calcd. for $\mathrm{C}_{28} \mathrm{H}_{26} \mathrm{~N}_{2} \mathrm{NaO}_{5} \mathrm{~S}[\mathrm{M}+\mathrm{Na}]^{+}: 525.1455$, found: 525.1458 .

ethyl 1-phenyl-3-((4-(trifluoromethyl)phenyl)sulfonyl)-3a,4-dihydro-3H-benzo[f]imidazo[1,2a]azepine-5-carboxylate (30):<smiles>CCOC(=O)C1=Cc2ccccc2N2C(c3ccccc3)=CN(S(=O)(=O)c3ccc(C(F)(F)F)cc3)CC12</smiles>

Yellow Solid (28\%, $25.2 \mathrm{mg}$ ); $\mathrm{R}_{\mathrm{f}}=0.4$ (hexane/EtOAc $=4: 1$ ); m.p. 285.3$286.2{ }^{\circ} \mathrm{C} ;{ }^{1} \mathrm{H}$ NMR $\left(300 \mathrm{MHz}, \mathrm{CDCl}_{3}\right) \delta 7.85-7.80(\mathrm{~m}, 3 \mathrm{H}), 7.61(\mathrm{~d}, J=8.4$ $\mathrm{Hz}, 2 \mathrm{H}$ ), 7.36 (dd, $J=7.9,0.6 \mathrm{~Hz}, 1 \mathrm{H}), 7.28-7.20(\mathrm{~m}, 3 \mathrm{H}), 7.10(\mathrm{dd}, J=8.0$, $1.6 \mathrm{~Hz}, 2 \mathrm{H}), 6.91(\mathrm{td}, J=7.5,1.0 \mathrm{~Hz}, 1 \mathrm{H}), 6.74-6.68(\mathrm{~m}, 1 \mathrm{H}), 6.20(\mathrm{~s}, 1 \mathrm{H})$, $5.27(\mathrm{~d}, J=7.7 \mathrm{~Hz}, 1 \mathrm{H}), 4.79(\mathrm{q}, J=4.2 \mathrm{~Hz}, 1 \mathrm{H}), 4.31(\mathrm{q}, J=7.1 \mathrm{~Hz}, 2 \mathrm{H})$, 3.24-3.08 (m, 2H), 1.37 (t, $J=7.2 \mathrm{~Hz}, 3 \mathrm{H}) ;{ }^{13} \mathrm{C} \mathrm{NMR}\left(75 \mathrm{MHz}, \mathrm{CDCl}_{3}\right) \delta$ $167.6,140.3,137.6,137.4,136.9,134.9,129.6,129.3,129.1,128.9,128.7$, $128.3,128.3,128.2,126.9,126.7,126.7,125.9,125.6,125.6,125.5,123.0$, 119.8, 108.0, 78.9, 61.4, 39.3, 14.3, 14.2; IR (liquid) v 3056, 2926, 1706, 1322, 1240, 1172, $712 \mathrm{~cm}^{-1}$; HRMS (ESI) m/z calcd. for $\mathrm{C}_{28} \mathrm{H}_{23} \mathrm{~F}_{3} \mathrm{~N}_{2} \mathrm{NaO}_{4} \mathrm{~S}$ [M+Na] $]^{+}$563.1223, found: 563.1226 .

hexyl 1-phenyl-3-tosyl-3a,4-dihydro-3H-benzo[f]imidazo[1,2-a]azepine-5-carboxylate (3p): 


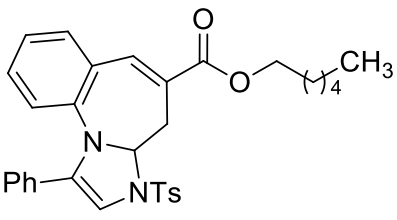

Yellow Solid $\left(69 \%, 74.4 \mathrm{mg}\right.$ ); $\mathrm{R}_{\mathrm{f}}=0.4$ (hexane/EtOAc $\left.=4: 1\right)$; m.p. 135.3$135.9^{\circ} \mathrm{C} ;{ }^{1} \mathrm{H}$ NMR $\left(300 \mathrm{MHz} \mathrm{CDCl}_{3}\right) \delta 7.80$ (s, 1H), 7.58 (d, J = 8.1 Hz, $2 \mathrm{H}), 7.35$ (d, J = 7.5 Hz, 1H), 7.26-7.20 (m, 3H), $7.11(\mathrm{~d}, J=7.5 \mathrm{~Hz}, 4 \mathrm{H})$, $6.88(\mathrm{t}, J=7.4 \mathrm{~Hz}, 1 \mathrm{H}), 6.71(\mathrm{t}, J=7.6 \mathrm{~Hz}, 1 \mathrm{H}), 6.20(\mathrm{~s}, 1 \mathrm{H}), 5.37(\mathrm{~d}, J=$ $7.9 \mathrm{~Hz}, 1 \mathrm{H}), 4.81(\mathrm{dd}, J=9.2,3.1 \mathrm{~Hz}, 1 \mathrm{H}), 4.24(\mathrm{t}, J=6.6 \mathrm{~Hz}, 2 \mathrm{H}), 3.22-$ $3.06(\mathrm{~m}, 2 \mathrm{H}), 2.30(\mathrm{~s}, 3 \mathrm{H}), 1.78-1.69(\mathrm{~m}, 2 \mathrm{H}), 1.45-1.34(\mathrm{~m}, 6 \mathrm{H}), 0.91(\mathrm{t}, J$ $=6.1 \mathrm{~Hz}, 3 \mathrm{H}) ;{ }^{13} \mathrm{C}$ NMR $\left(75 \mathrm{MHz}, \mathrm{CDCl}_{3}\right) \delta 167.8,144.3,140.6,137.6,136.5,134.7,130.6,129.1,128.9$, 128.7, 128.6, 128.4, 127.8, 126.6, 125.9, 122.5, 120.3, 109.1, 78.8, 65.5, 39.4, 31.4, 28.6, 25.7, 22.5, 21.4, 14.0; IR (solid) v 3117, 2961, 1704, 1236, 1188, $679 \mathrm{~cm}^{-1} ;$ HRMS (ESI) m/z calcd. for $\mathrm{C}_{32} \mathrm{H}_{34} \mathrm{~N}_{2} \mathrm{NaO}_{4} \mathrm{~S}[\mathrm{M}+\mathrm{Na}]^{+}:$565.2131, found: 565.2126.

benzyl 1-phenyl-3-tosyl-3a,4-dihydro-3H-benzo[f]imidazo[1,2-a]azepine-5-carboxylate (3q):<smiles>O=C(OCc1ccccc1)C1=Cc2ccccc2-n2c(-c3ccccc3)c[n+]([S-])c2C1</smiles>
Yellow Solid $\left(76 \%, 83.2 \mathrm{mg}\right.$ ); $\mathrm{R}_{\mathrm{f}}=0.4$ (hexane/EtOAc $=4: 1$ ); m.p. 137.4$138.0^{\circ} \mathrm{C} ;{ }^{1} \mathrm{H}$ NMR $\left(300 \mathrm{MHz}, \mathrm{CDCl}_{3}\right) \delta 7.84(\mathrm{~s}, 1 \mathrm{H}), 7.57(\mathrm{~d}, J=8.1 \mathrm{~Hz}, 2 \mathrm{H})$, 7.45-7.32 (m, 6H), 7.25-7.19 (m, 3H), $7.10(\mathrm{~d}, J=6.8 \mathrm{~Hz}, 4 \mathrm{H}), 6.87(\mathrm{t}, J=7.4$ $\mathrm{Hz}, 1 \mathrm{H}), 6.70(\mathrm{t}, J=7.6 \mathrm{~Hz}, 1 \mathrm{H}), 6.18(\mathrm{~s}, 1 \mathrm{H}), 5.38(\mathrm{~d}, J=8.1 \mathrm{~Hz}, 1 \mathrm{H}), 5.33-$ $5.25(\mathrm{~m}, 2 \mathrm{H}), 4.81(\mathrm{~d}, J=6.6 \mathrm{~Hz}, 1 \mathrm{H}), 3.25-3.08(\mathrm{~m}, 2 \mathrm{H}), 2.29(\mathrm{~s}, 3 \mathrm{H}) ;{ }^{13} \mathrm{C}$ NMR $\left(75 \mathrm{MHz}, \mathrm{CDCl}_{3}\right) \delta 167.6,144.2,140.7,138.2,136.5,135.9,134.8$, $130.7,130.7,129.1,128.9,128.7,128.6,128.2,128.0,127.8,126.6,125.8,122.5,120.3,109.1,78.8$, 67.0, 39.5, 21.4; IR (solid) v 3116, 1712, 1698, 1352, 1238, 1194, 1155, $681 \mathrm{~cm}^{-1}$; HRMS (ESI) m/z calcd. for $\mathrm{C}_{33} \mathrm{H}_{29} \mathrm{~N}_{2} \mathrm{O}_{4} \mathrm{~S}[\mathrm{M}+\mathrm{H}]+:$ 549.1843, found: 549.1849 .

isopropyl 1-phenyl-3-tosyl-3a,4-dihydro-3H-benzo[f]imidazo[1,2-a]azepine-5-carboxylate (3r):<smiles>CC(C)OC(=O)C1=Cc2ccccc2N2C(c3ccccc3)=C[N+](C)(S)C2C1</smiles>
Yellow Solid $(77 \%, 77.2 \mathrm{mg}) ; \mathrm{R}_{\mathrm{f}}=0.4$ (hexane/EtOAc $\left.=4: 1\right) ;$ m.p. $134.1-134.7^{\circ} \mathrm{C}$; ${ }^{1} \mathrm{H}$ NMR $\left(300 \mathrm{MHz}_{\mathrm{CDCl}}\right) \delta 7.78(\mathrm{~s}, 1 \mathrm{H}), 7.58(\mathrm{~d}, \mathrm{~J}=8.3 \mathrm{~Hz}, 2 \mathrm{H}), 7.35(\mathrm{~d}, \mathrm{~J}=$ $7.5 \mathrm{~Hz}, 1 \mathrm{H}), 7.26-7.20(\mathrm{~m}, 3 \mathrm{H}), 7.11(\mathrm{~d}, J=7.9 \mathrm{~Hz}, 4 \mathrm{H}), 6.88(\mathrm{t}, J=7.4 \mathrm{~Hz}, 1 \mathrm{H})$, $6.70(\mathrm{t}, J=7.3 \mathrm{~Hz}, 1 \mathrm{H}), 6.19(\mathrm{~s}, 1 \mathrm{H}), 5.37(\mathrm{~d}, J=7.9 \mathrm{~Hz}, 1 \mathrm{H}), 5.23-5.10(\mathrm{~m}, 1 \mathrm{H})$, $4.80(\mathrm{dd}, J=9.1,3.4 \mathrm{~Hz}, 1 \mathrm{H}), 3.22-3.05(\mathrm{~m}, 2 \mathrm{H}), 2.30(\mathrm{~s}, 3 \mathrm{H}), 1.36-1.32(\mathrm{~m}, 6 \mathrm{H})$; ${ }^{13} \mathrm{C}$ NMR $\left(75 \mathrm{MHz}, \mathrm{CDCl}_{3}\right) \delta$ 167.3, 144.3, 140.6, 137.4, 136.5, 134.6, 130.7, 129.1, 128.9, 128.8, 128.7, 128.6, 128.3, 127.8, 126.7, 126.0, 122.5, 120.3, 109.0, 78.9, 68.7, 39.5, 21.9, 21.9, 21.4; IR (solid) v 2983, 1702, 1348, 1162, 678, $661 \mathrm{~cm}^{-1} ; \mathrm{HRMS}$ (ESI) m/z calcd. for $\mathrm{C}_{29} \mathrm{H}_{29} \mathrm{~N}_{2} \mathrm{O}_{4} \mathrm{~S}[\mathrm{M}+\mathrm{H}]^{+}: 501.1843$, found: 501.1845 .

cyclohexyl 1-phenyl-3-tosyl-3a,4-dihydro-3H-benzo[f]imidazo[1,2-a]azepine-5-carboxylate (3s):

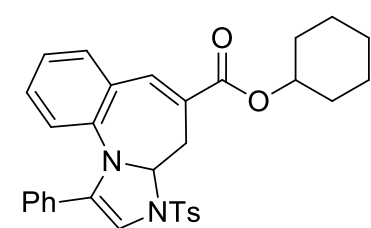
Yellow Solid $\left(85 \%, 91.7 \mathrm{mg}\right.$ ); $\mathrm{R}_{\mathrm{f}}=0.4$ (hexane/EtOAc $=4: 1$ ); m.p. 118.6$119.5^{\circ} \mathrm{C} ;{ }^{1} \mathrm{H}$ NMR $\left(400 \mathrm{MHz}, \mathrm{CDCl}_{3}\right) \delta 7.80(\mathrm{~s}, 1 \mathrm{H}), 7.58(\mathrm{~d}, J=8.0 \mathrm{~Hz}, 2 \mathrm{H})$, $7.35(\mathrm{~d}, J=7.5 \mathrm{~Hz}, 1 \mathrm{H}), 7.27-7.20(\mathrm{~m}, 3 \mathrm{H}), 7.12(\mathrm{~d}, J=7.8 \mathrm{~Hz}, 3 \mathrm{H}), 6.88(\mathrm{t}$, $J=7.4 \mathrm{~Hz}, 1 \mathrm{H}), 6.70(\mathrm{t}, J=7.5 \mathrm{~Hz}, 1 \mathrm{H}), 6.20(\mathrm{~s}, 1 \mathrm{H}), 5.37(\mathrm{~d}, J=8.0 \mathrm{~Hz}$, $1 \mathrm{H}), 4.97-4.92(\mathrm{~m}, 1 \mathrm{H}), 4.81(\mathrm{dd}, J=9.4,3.0 \mathrm{~Hz}, 1 \mathrm{H}), 3.20-3.07(\mathrm{~m}, 2 \mathrm{H})$, 2.38-2.30 (m, 3H), 1.91-1.77 (m, 4H), 1.57-1.26 (m, 7H); ${ }^{13} \mathrm{C}$ NMR (100 MHz, $\left.\mathrm{CDCl}_{3}\right) \delta 167.1,144.3,140.6,137.4,136.6,134.6,130.5,129.1,128.9,128.8,128.7,128.6,128.3$, 127.8, 126.6, 125.9, 122.5, 120.2, 108.9, 78.9, 73.3, 39.4, 31.6, 31.5, 25.4, 23.6, 21.4; IR (solid) v 2933, 1704, 1245, 1198, 1158, $678 \mathrm{~cm}^{-1}$; HRMS (ESI) m/z calcd. for $\mathrm{C}_{32} \mathrm{H}_{33} \mathrm{~N}_{2} \mathrm{O}_{4} \mathrm{~S}[\mathrm{M}+\mathrm{H}]^{+}: 541.2156$, found: 541.2162.

tert-butyl 1-phenyl-3-tosyl-3a,4-dihydro-3H-benzo[f]imidazo[1,2-a]azepine-5-carboxylate (3t): Yellow Solid $\left(75 \%, 77.4 \mathrm{mg}\right.$ ); $\mathrm{R}_{\mathrm{f}}=0.4$ (hexane/EtOAc $=4: 1$ ); m.p. 130.2$131.2{ }^{\circ} \mathrm{C} ;{ }^{1} \mathrm{H}$ NMR $\left(400 \mathrm{MHz}, \mathrm{CDCl}_{3}\right) \delta 7.73(\mathrm{~s}, 1 \mathrm{H}), 7.58(\mathrm{~d}, J=8.2 \mathrm{~Hz}, 2 \mathrm{H})$,<smiles>CC(C)(C)OC(=O)C1=Cc2ccccc2N2C(c3ccccc3)=C[NH+]([SH+])CC2C1</smiles>
$7.33(\mathrm{~d}, J=7.5 \mathrm{~Hz}, 1 \mathrm{H}), 7.26-7.10(\mathrm{~m}, 7 \mathrm{H}), 6.87(\mathrm{t}, J=7.3 \mathrm{~Hz}, 1 \mathrm{H}), 6.69$ (t, J $=7.2 \mathrm{~Hz}, 1 \mathrm{H}), 6.19(\mathrm{~s}, 1 \mathrm{H}), 5.36(\mathrm{~d}, J=7.8 \mathrm{~Hz}, 1 \mathrm{H}), 4.81-4.78(\mathrm{~m}, 1 \mathrm{H}), 3.15-$ $3.04(\mathrm{~m}, 2 \mathrm{H}), 2.30$ (s, 3H), $1.56(\mathrm{~s}, 9 \mathrm{H}) ;{ }^{13} \mathrm{C} \mathrm{NMR}\left(100 \mathrm{MHz}, \mathrm{CDCl}_{3}\right) \delta$ 166.9, 144.3, 140.6, 136.8, 136.6, 134.6, 130.6, 129.9, 129.1, 128.9, 128.7, 128.6, 
128.2, 127.8, 126.7, 126.0, 122.5, 120.2, 108.9, 81.2, 78.9, 39.6, 28.1, 21.4; IR (solid) v 3067, 1698, 1488, 1359, 1252, 1167, $668 \mathrm{~cm}^{-1}$; HRMS (ESI) m/z calcd. for $\mathrm{C}_{30} \mathrm{H}_{31} \mathrm{~N}_{2} \mathrm{O}_{4} \mathrm{~S}[\mathrm{M}+\mathrm{H}]^{+}: 515.1999$, found: 515.2003.

4-methoxyphenyl 1-phenyl-3-tosyl-3a,4-dihydro-3H-benzo[f]imidazo[1,2-a]azepine-5carboxylate $(3 u)$ :<smiles>COc1ccc(OC(=O)C2=Cc3ccccc3N3C(c4ccccc4)=C[N+](S)(Cc4ccccc4)C3C2)cc1</smiles>

Yellow Solid $(50 \%, 56.9 \mathrm{mg}) ; \mathrm{R}_{\mathrm{f}}=0.4$ (hexane/EtOAc $=4: 1$ ); m.p. 128.1-128.9 ${ }^{\circ} \mathrm{C} ;{ }^{1} \mathrm{H}$ NMR $\left(300 \mathrm{MHz}, \mathrm{CDCl}_{3}\right) \delta 7.98(\mathrm{~s}, 1 \mathrm{H}), 7.60(\mathrm{~d}, J=$ $8.1 \mathrm{~Hz}, 2 \mathrm{H}), 7.39(\mathrm{~d}, J=7.7 \mathrm{~Hz}, 1 \mathrm{H}), 7.25-7.08(\mathrm{~m}, 9 \mathrm{H}), 6.95-6.88(\mathrm{~m}$, $3 \mathrm{H}), 6.75(\mathrm{t}, J=7.7 \mathrm{~Hz}, 1 \mathrm{H}), 6.22(\mathrm{~s}, 1 \mathrm{H}), 5.43(\mathrm{~d}, J=7.9 \mathrm{~Hz}, 1 \mathrm{H})$, 4.90-4.87 (m, 1H), $3.82(\mathrm{~s}, 3 \mathrm{H}), 3.38-3.20(\mathrm{~m}, 2 \mathrm{H}), 2.30(\mathrm{~s}, 3 \mathrm{H}) ;{ }^{13} \mathrm{C}$ NMR $\left(75 \mathrm{MHz}, \mathrm{CDCl}_{3}\right) \delta 166.7,157.2,144.5,144.3,140.9,139.3$, 136.4, 135.0, 130.8, 129.1, 129.0, 128.9, 128.7, 128.6, 127.8, 127.6, 126.7, 125.6, 122.6, 122.3, 120.4, 114.5, 109.3, 78.9, 55.6, 39.7, 21.4; IR (solid) v 1722, 1503, 1158 , $760,694,660 \mathrm{~cm}^{-1}$; HRMS (ESI) m/z calcd. for $\mathrm{C}_{33} \mathrm{H}_{28} \mathrm{~N}_{2} \mathrm{NaO}_{5} \mathrm{~S}$ [M+Na]+: 587.1611, found: 587.1618.

\section{B. Products of Application Reactions}

\section{ethyl 1-phenyl-4H-benzo[f] imidazo[1,2-a]azepine-5-carboxylate (4):}<smiles>CCOC(=O)C1=Cc2ccccc2-n2c(-c3ccccc3)cnc2C1</smiles>

White Solid (99\%, $32.7 \mathrm{mg}) ; \mathrm{R}_{\mathrm{f}}=0.5$ (EtOAc); m.p. 66.6-67.5 ${ }^{\circ} \mathrm{C} ;{ }^{1} \mathrm{H} \mathrm{NMR}(300 \mathrm{MHz}$, $\left.\mathrm{CDCl}_{3}\right) \delta 7.84(\mathrm{~s}, 1 \mathrm{H}), 7.52(\mathrm{~d}, J=7.7 \mathrm{~Hz}, 1 \mathrm{H}), 7.34-7.23(\mathrm{~m}, 4 \mathrm{H}), 7.17-7.12(\mathrm{~m}$, $2 \mathrm{H}), 7.04-7.01(\mathrm{~m}, 2 \mathrm{H}), 6.87(\mathrm{~d}, J=8.1 \mathrm{~Hz}, 1 \mathrm{H}), 4.46-4.32(\mathrm{~m}, 3 \mathrm{H}), 3.05(\mathrm{~d}, J=$ $13.6 \mathrm{~Hz}, 1 \mathrm{H}), 1.39$ (t, $J=7.2 \mathrm{~Hz}, 3 \mathrm{H}) ;{ }^{13} \mathrm{C} \mathrm{NMR}\left(75 \mathrm{MHz}, \mathrm{CDCl}_{3}\right) \delta 165.2,152.1$, $136.7,134.9,133.8,132.9,130.9,130.1,129.5,129.1,128.8,128.4,127.7,127.3$, 126.7, 126.3, 61.5, 26.4, 14.2; IR (solid) v 2984, 1704, 1450, 1181, 754, $695 \mathrm{~cm}^{-1}$; HRMS (ESI) $\mathrm{m} / \mathrm{z}$ calcd. for $\mathrm{C}_{21} \mathrm{H}_{19} \mathrm{~N}_{2} \mathrm{O}_{2}[\mathrm{M}+\mathrm{H}]^{+}: 331.1441$, found: 331.1445 .

ethyl 1-phenyl-5,6-dihydro-4H-benzo[f]imidazo[1,2-a]azepine-5-carboxylate (5):<smiles>CCOC(=O)C1Cc2ccccc2-n2c(-c3ccccc3)cnc2C1</smiles>
Colourless oil $(98 \%, 32.6 \mathrm{mg}) ; \mathrm{R}_{\mathrm{f}}=0.4$ (hexane/EtOAc $\left.=2: 1\right) ;{ }^{1} \mathrm{H} \mathrm{NMR}(300 \mathrm{MHz}$, $\left.\mathrm{CDCl}_{3}\right) \delta 7.42(\mathrm{~d}, J=7.3 \mathrm{~Hz}, 1 \mathrm{H}), 7.30-7.24(\mathrm{~m}, 4 \mathrm{H}), 7.16-7.10(\mathrm{~m}, 4 \mathrm{H}), 6.73(\mathrm{~d}, J$ $=7.9 \mathrm{~Hz}, 1 \mathrm{H}), 4.28-4.11(\mathrm{~m}, 2 \mathrm{H}), 3.49-3.40(\mathrm{~m}, 1 \mathrm{H}), 3.34-2.91(\mathrm{~m}, 3 \mathrm{H}), 2.73-2.64$ $(\mathrm{m}, 1 \mathrm{H}), 1.34-1.26(\mathrm{~m}, 3 \mathrm{H}) ;{ }^{13} \mathrm{C} \mathrm{NMR}\left(75 \mathrm{MHz}, \mathrm{CDCl}_{3}\right) \delta 173.2,136.4,136.2,133.4$, 132.5, 131.2, 130.1, 129.7, 128.4, 127.8, 127.5, 127.4, 127.3, 125.5, 125.3, 60.9, 46.4, 32.3, 27.4, 14.2; IR (liquid) v 2977, 2924, 1731, 1184, $761 \mathrm{~cm}^{-1}$; HRMS (ESI) $\mathrm{m} / \mathrm{z}$ calcd. for $\mathrm{C}_{21} \mathrm{H}_{21} \mathrm{~N}_{2} \mathrm{O}_{2}[\mathrm{M}+\mathrm{H}]^{+}: 333.1598$, found: 333.1598 .

\section{(1-phenyl-4H-benzo[f]imidazo[1,2-a]azepin-5-yl)methanol (6):}<smiles>OCC1=Cc2ccccc2-n2c(-c3ccccc3)cnc2C1</smiles>

White Solid $(99 \%, 57.2 \mathrm{mg}) ; \mathrm{R}_{\mathrm{f}}=0.3$ (EtOAc); m.p. $177.9-178.3^{\circ} \mathrm{C} ;{ }^{1} \mathrm{H}$ NMR $(300$ $\left.\mathrm{MHz}, \mathrm{CDCl}_{3}\right) \delta 7.43(\mathrm{~d}, J=7.7 \mathrm{~Hz}, 1 \mathrm{H}), 7.29-7.22(\mathrm{~m}, 4 \mathrm{H}), 7.06-7.00(\mathrm{~m}, 4 \mathrm{H})$, $6.84(\mathrm{~d}, J=8.3 \mathrm{~Hz}, 1 \mathrm{H}), 6.74(\mathrm{~s}, 1 \mathrm{H}), 4.45(\mathrm{~s}, 2 \mathrm{H}), 3.59(\mathrm{~d}, J=14.3 \mathrm{~Hz}, 1 \mathrm{H}), 3.08$ $(\mathrm{d}, J=12.8 \mathrm{~Hz}, 1 \mathrm{H}) ;{ }^{13} \mathrm{C}$ NMR $\left(75 \mathrm{MHz}, \mathrm{CDCl}_{3}\right) \delta 152.1,144.1,133.8,133.6,131.3$, 130.2, 130.1, 128.4, 128.1, 127.8, 127.3, 126.6, 126.5, 126.3, 122.5, 65.4, 28.5; IR (solid) v 3049, 1512, 1452, 760, 730, $691 \mathrm{~cm}^{-1}$; HRMS (ESI) m/z calcd. for $\mathrm{C}_{19} \mathrm{H}_{17} \mathrm{~N}_{2} \mathrm{O}[\mathrm{M}+\mathrm{H}]^{+}: 289.1335$, found: 289.1340 .

\section{5-(chloromethyl)-1-phenyl-4H-benzo[f]imidazo[1,2-a]azepine (7):}<smiles>ClCC1=Cc2ccccc2-n2c(-c3ccccc3)cnc2C1</smiles>

White Solid (45\%, $13.7 \mathrm{mg}) ; \mathrm{R}_{\mathrm{f}}=0.4$ (EtOAc); m.p. 260.9-262. $1{ }^{\circ} \mathrm{C} ;{ }^{1} \mathrm{H}$ NMR $(300$ $\left.\mathrm{MHz}, \mathrm{CDCl}_{3}\right) \delta 7.43(\mathrm{~d}, J=7.7 \mathrm{~Hz}, 1 \mathrm{H}), 7.30-7.23(\mathrm{~m}, 4 \mathrm{H}), 7.11(\mathrm{~s}, 1 \mathrm{H}), 7.08-7.01$ $(\mathrm{m}, 3 \mathrm{H}), 6.85(\mathrm{~d}, J=8.1 \mathrm{~Hz}, 1 \mathrm{H}), 6.78(\mathrm{~s}, 1 \mathrm{H}), 4.36(\mathrm{~d}, J=9.0 \mathrm{~Hz}, 2 \mathrm{H}), 3.73(\mathrm{~d}, J=$ $14.1 \mathrm{~Hz}, 1 \mathrm{H}), 3.15(\mathrm{~d}, J=14.1 \mathrm{~Hz}, 1 \mathrm{H}) ;{ }^{13} \mathrm{C}$ NMR $\left(75 \mathrm{MHz}, \mathrm{CDCl}_{3}\right) \delta 151.6,139.1$, 134.2, 133.7, 130.2, 130.2, 128.9, 128.4, 127.8, 127.4, 127.3, 126.7, 126.5, 126.3, 48.7, 29.6; IR (solid) v 3069, 1712, 1539, 1369, 758, $695 \mathrm{~cm}^{-1}$; HRMS (ESI) m/z calcd. for $\mathrm{C}_{19} \mathrm{H}_{16} \mathrm{ClN}_{2}[\mathrm{M}+\mathrm{H}]+$ : 307.0997 , found: 307.1004 . 


\section{III-3. Products of Mechanistic Experiments}

ethyl 3a-deuterium-1-phenyl-3-tosyl-3a,4-dihydro-3H-benzo[f]imidazo[1,2-a]azepine-5carboxylate (3a $\left.a^{1}-D\right)$ :<smiles>[2H]C12C(C(=O)OCC)=Cc3ccccc3N1C(c1ccccc1)=C[N+]2([SH3+])c1ccccc1</smiles>

Yellow Solid $\left(70 \%, 68.0 \mathrm{mg}\right.$ ); $\mathrm{R}_{\mathrm{f}}=0.4$ (hexane/EtOAc $\left.=4: 1\right)$; m.p. $138.9-140.1{ }^{\circ} \mathrm{C}$; ${ }^{1} \mathrm{H} \mathrm{NMR}\left(300 \mathrm{MHz}, \mathrm{CDCl}_{3}\right) \delta 7.80(\mathrm{~s}, 1 \mathrm{H}), 7.58(\mathrm{~d}, J=8.1 \mathrm{~Hz}, 2 \mathrm{H}), 7.34(\mathrm{~d}, J=7.7$ $\mathrm{Hz}, 1 \mathrm{H}), 7.25-7.19(\mathrm{~m}, 3 \mathrm{H}), 7.11(\mathrm{~d}, J=6.8 \mathrm{~Hz}, 4 \mathrm{H}), 6.88(\mathrm{t}, \mathrm{J}=7.4 \mathrm{~Hz}, 1 \mathrm{H}), 6.70(\mathrm{t}$, $J=7.6 \mathrm{~Hz}, 1 \mathrm{H}), 6.19(\mathrm{~s}, 1 \mathrm{H}), 5.38(\mathrm{~d}, J=7.9 \mathrm{~Hz}, 1 \mathrm{H}), 4.30(\mathrm{q}, J=7.1 \mathrm{~Hz}, 2 \mathrm{H}), 3.22-$ $3.07(\mathrm{~m}, 1.6 \mathrm{H}), 2.29(\mathrm{~s}, 3 \mathrm{H}), 1.36(\mathrm{t}, J=7.1 \mathrm{~Hz}, 3 \mathrm{H}) ;{ }^{13} \mathrm{C} \mathrm{NMR}\left(75 \mathrm{MHz}, \mathrm{CDCl}_{3}\right) \delta$ $167.8,144.2,140.6,137.7,137.6,136.5,134.7,130.7,129.1,128.9,128.7,128.6$, 128.4, 128.4, 128.3, 127.8, 126.6, 125.8, 122.5, 120.3, 109.1, 61.2, 39.4, 21.4, 14.3; IR (liquid) v 3060, $2981,1704,1363,1167,677,596 \mathrm{~cm}^{-1}$; HRMS (ESI) m/z calcd. for $\mathrm{C}_{28} \mathrm{H}_{25} \mathrm{DN}_{2} \mathrm{NaO}_{4} \mathrm{~S}[\mathrm{M}+\mathrm{Na}]^{+}: 510.1568$, found: 510.1577 .

(Z)-tert-butyl 2-(1-((Z)-2-(4-methylphenylsulfonamido)-1-phenylvinyl)quinolin-4(1H)ylidene)acetate (9):

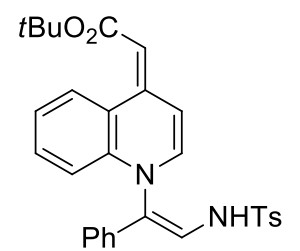

Yellow Solid (6\%, 5.9mg); $R_{f}=0.2$ (EtOAc); m.p. 258.8-260.3 ${ }^{\circ} \mathrm{C} ;{ }^{1} \mathrm{H}$ NMR $(400$ $\left.\mathrm{MHz}, \mathrm{CDCl}_{3}\right) \delta 7.91-7.88(\mathrm{~m}, 1 \mathrm{H}), 7.71(\mathrm{t}, J=7.5 \mathrm{~Hz}, 3 \mathrm{H}), 7.33(\mathrm{~d}, J=8.0 \mathrm{~Hz}, 2 \mathrm{H})$, 7.30-7.28 (m, 1H), $7.24(\mathrm{~m}, 1 \mathrm{H}), 7.19-7.06(\mathrm{~m}, 6 \mathrm{H}), 6.71-6.68(\mathrm{~m}, 1 \mathrm{H}), 6.34(\mathrm{~d}, J=$ $8.0 \mathrm{~Hz}, 1 \mathrm{H}), 5.78(\mathrm{~s}, 1 \mathrm{H}), 2.46(\mathrm{~s}, 3 \mathrm{H}), 1.54(\mathrm{~s}, 9 \mathrm{H}) ;{ }^{13} \mathrm{C} \mathrm{NMR}\left(100 \mathrm{MHz}, \mathrm{CDCl}_{3}\right) \delta$ 168.2, 144.6, 143.8, 137.2, 136.5, 134.7, 133.9, 130.7, 130.2, 129.1, 128.3, 126.7, 124.3, 124.0, 123.9, 123.0, 122.3, 122.0, 115.7, 106.5, 96.0, 78.6, 28.6, 21.6; IR (liquid) $v$ 2924, 2853, 1560, 1456, 1166, $1131 \mathrm{~cm}^{-1}$; HRMS (ESI) m/z calcd. for $\mathrm{C}_{30} \mathrm{H}_{31} \mathrm{~N}_{2} \mathrm{O}_{4} \mathrm{~S}[\mathrm{M}+\mathrm{H}]^{+}$: 515.1999, found: 515.2004 . 


\section{X-ray Crystal Data (3a)}

Preparation: To the mixture of compound $3 \mathbf{a}(5.0 \mathrm{mg})$ in $\mathrm{CH}_{2} \mathrm{Cl}_{2}(3 \mathrm{~mL}), 1.0 \mathrm{~mL}$ of hexane was slowly added at room temperature. And then, the solvent slowly diffused. The single crystal was obtained after five days.

The structure in Figure S1 showed the absolute configuration of $\mathbf{3 a}$.

The CCDC number is 2060953. These details can be obtained free of charge via www.ccdc.com.ac.uk/data_request/cif from the Cambridge Crystallo- graphic Data Centre.

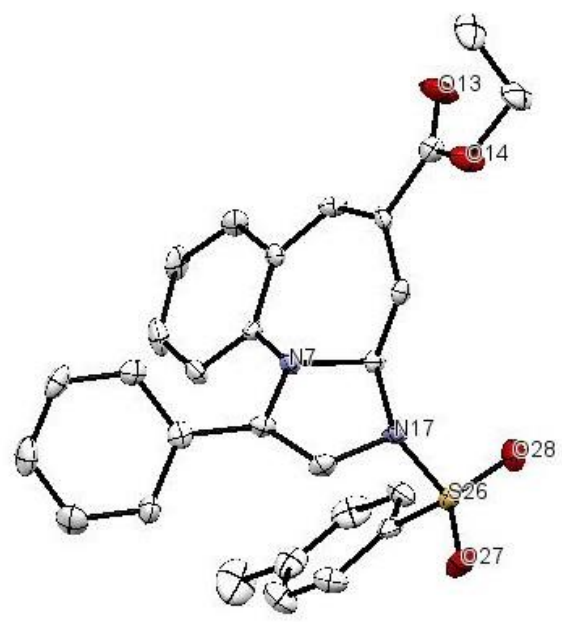

Figure S1. X-Ray Structure of 3a (50\% probability level)

Table S2. Table 1. Crystal data and structure refinement for 3a.

Empirical formula

Formula weight

Temperature

Wavelength

Crystal system

Space group

Unit cell dimensions

Volume

Z

Density (calculated)

Absorption coefficient

$\mathrm{F}(000)$

\section{$\mathrm{C}_{28} \mathrm{H}_{26} \mathrm{~N}_{2} \mathrm{O}_{4} \mathrm{~S}$}

486.57

173(2) K

$0.71073 \AA$

Orthorhombic

Pbca

$\begin{array}{ll}\mathrm{a}=7.8008(4) \AA & \alpha=90^{\circ} \\ \mathrm{b}=19.1685(10) \AA & \beta=90^{\circ} \\ \mathrm{c}=31.8776(15) \AA & \gamma=90^{\circ}\end{array}$

4766.6(4) $\AA^{3}$

8

$1.356 \mathrm{Mg} / \mathrm{m}^{3}$

$0.174 \mathrm{~mm}^{-1}$

2048 
Crystal size

Theta range for data collection

Index ranges

Reflections collected

Independent reflections

Completeness to theta $=24.998^{\circ}$

Absorption correction

Max. and min. transmission

Refinement method

Data / restraints / parameters

Goodness-of-fit on $\mathrm{F}^{2}$

Final $\mathrm{R}$ indices [I $>2 \operatorname{sigma}(\mathrm{I})]$

$\mathrm{R}$ indices (all data)

Largest diff. peak and hole
$0.189 \times 0.067 \times 0.029 \mathrm{~mm}^{3}$

2.480 to $24.998^{\circ}$.

$-9<=\mathrm{h}<=9,-22<=\mathrm{k}<=22,-37<=\mathrm{l}<=37$

85726

$4183[\mathrm{R}(\mathrm{int})=0.1561]$

$99.7 \%$

Semi-empirical from equivalents

0.7107 and 0.6609

Full-matrix least-squares on $\mathrm{F}^{2}$

4183 / 6 / 329

1.332

$\mathrm{R} 1=0.1203, \mathrm{wR} 2=0.1959$

$\mathrm{R} 1=0.1344, \mathrm{wR} 2=0.2014$

0.442 and $-0.414 \mathrm{e} \cdot \AA^{-3}$

\section{Reference}

S1. (a) Lee, D. J.; Han, H. S.; Shin, J.; Yoo, E. J. Multicomponent [5 + 2] Cycloaddition Reaction for the Synthesis of 1,4-Diazepines: Isolation and Reactivity of Azomethine Ylides. J. Am. Chem. Soc., 2014, 136, 11606. (b) Lee, J. Y.; Shim, J. Y.; Kim, H. K.; Ko, D.; Baik, M.-H.; Yoo, E. J. 3,5Diarylimidazo[1,2-a]pyridines as Color-Tunable Fluorophores. J. Org. Chem., 2017, 82, 4352. (c) De, N.; Song, C. E.; Ryu, D. H.; Yoo, E. J. Gold-catalyzed [5 + 2] cycloaddition of quinolinium zwitterions and allenamides as an efficient route to fused 1,4-diazepines. Chem. Commun., 2018, 54, 6911. (d) De, N.; Ko, D.; Baek, S.-y.; Oh, C.; Kim, J.; Baik, M.-H.; Yoo, E. J. Cu(I)-Catalyzed Enantioselective [5 + 1] Cycloaddition of $\mathrm{N}$-Aromatic Compounds and Alkynes via Chelating-Assisted 1,2-Dearomative Addition. ACS Catal., 2020, 10, 10905.

S2. Mao, H.; Lin, A.; Shi, Y.; Mao, X.; Zhu, C.; Li, W.; Hu, H.; Cheng, Y.; Zhu, C. Construction of Enantiomerically Enriched Diazo Compounds Using Diazo Esters as Nucleophiles: Chiral Lewis Base Catalysis. Angew. Chem., Int. Ed. 2013, 52, 6288.

S3. Ahmed, W.; Zhang, S.; Yu, X.; Feng, X.; Yamamoto, Y.; Bao, M. Direct Carbohydroxylation of Arylalkenes with Allylic Alcohols through Cooperative Catalysis of Copper, Silver, and Brønsted Acid. Angew. Chem., Int. Ed. 2019, 58, 2495. 
VI. Copies of Spectral Data of Compounds Obtained in this Study

(Z)-(2-phenyl-2-(2-deuterium-quinolin-1-ium-1-yl)vinyl)(tosyl)amide (1a-D):

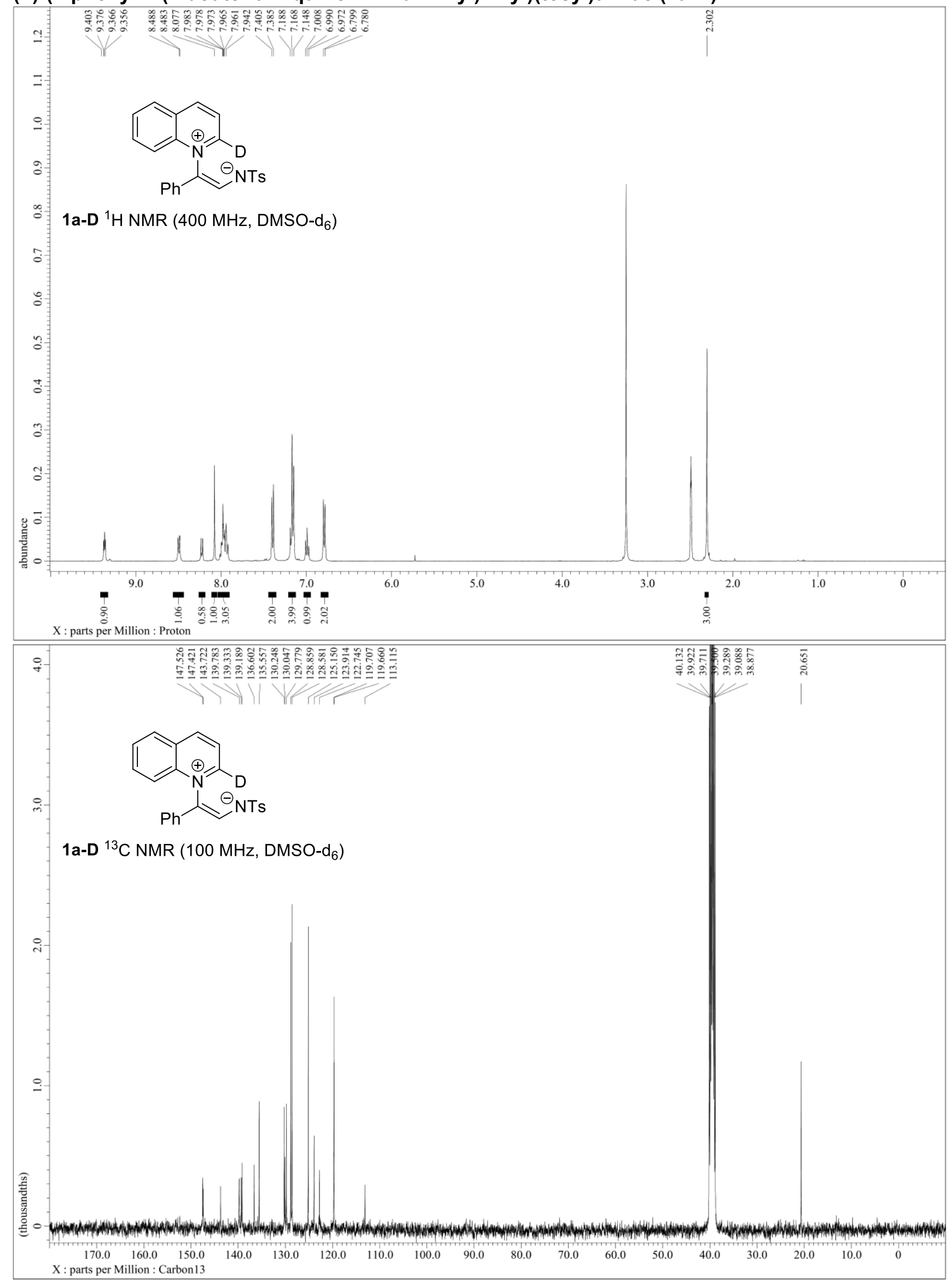


ethyl 1-phenyl-3-tosyl-3a,4-dihydro-3H-benzo[f]imidazo[1,2-a]azepine-5-carboxylate (3a):

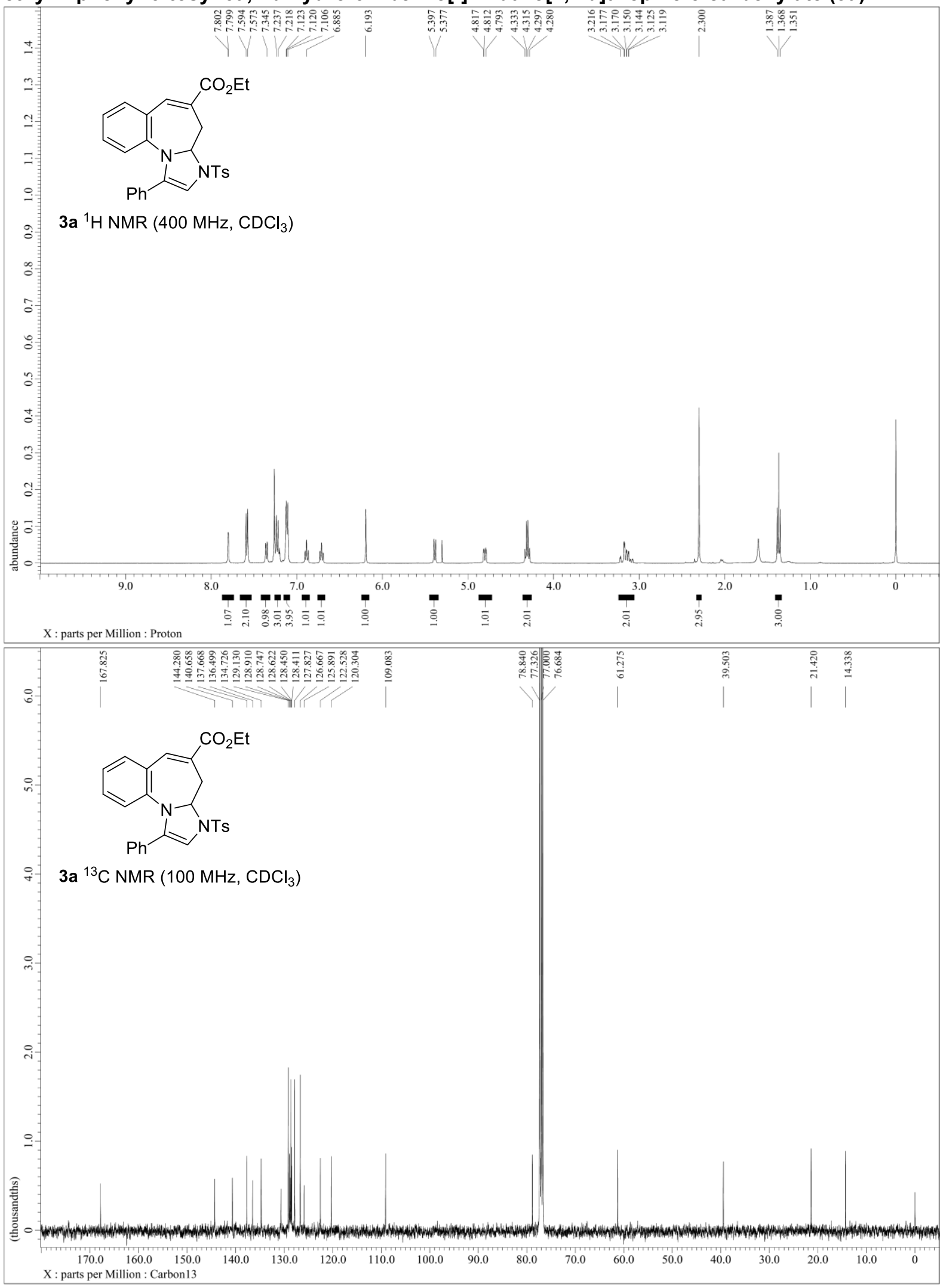


ethyl 7-bromo-1-phenyl-3-tosyl-3a,4-dihydro-3H-benzo[f]imidazo[1,2-a]azepine-5-carboxylate (3b):
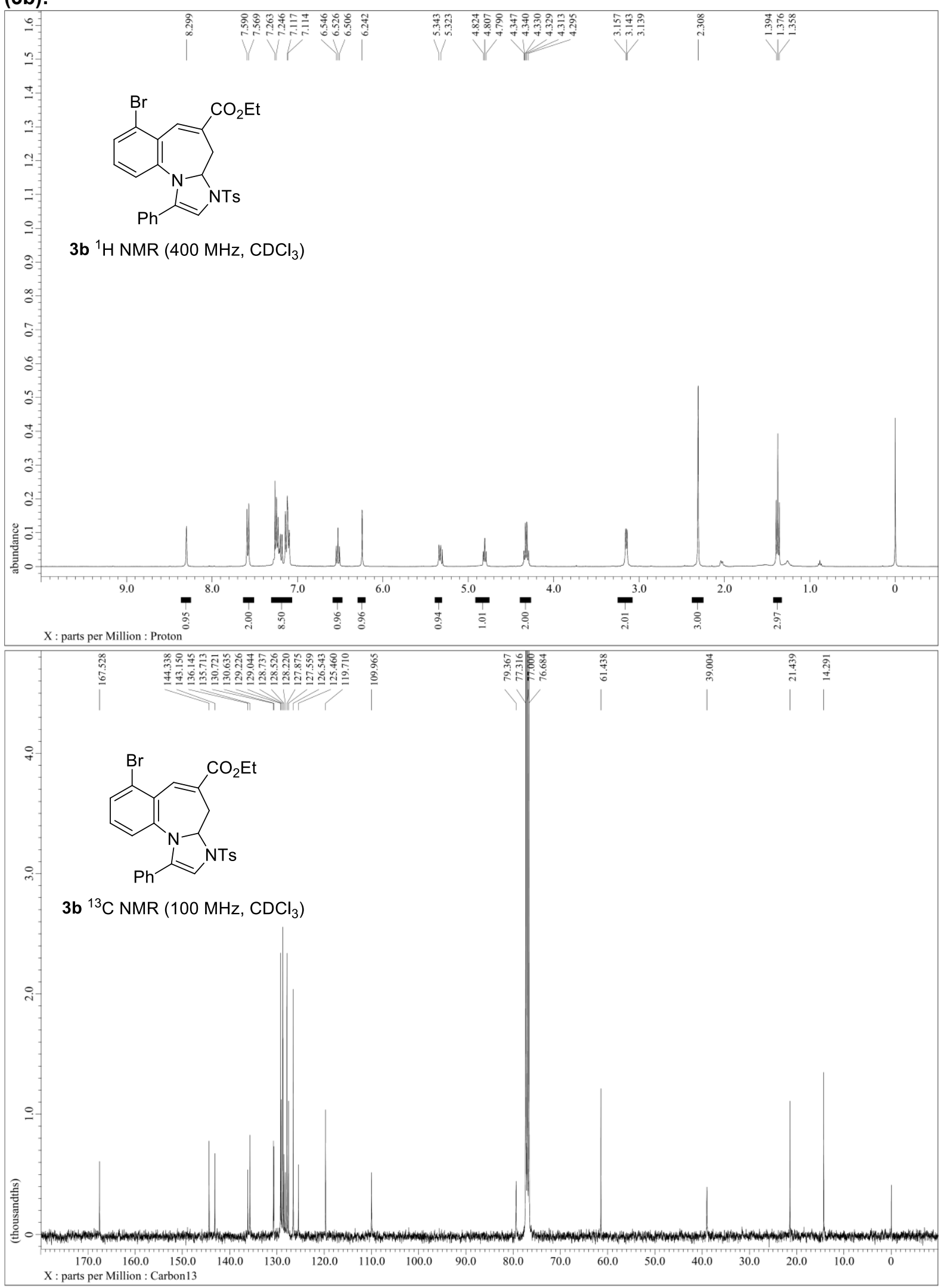
ethyl 8-methoxy-1-phenyl-3-tosyl-3a,4-dihydro-3H-benzo[f]imidazo[1,2-a]azepine-5-carboxylate (3c):

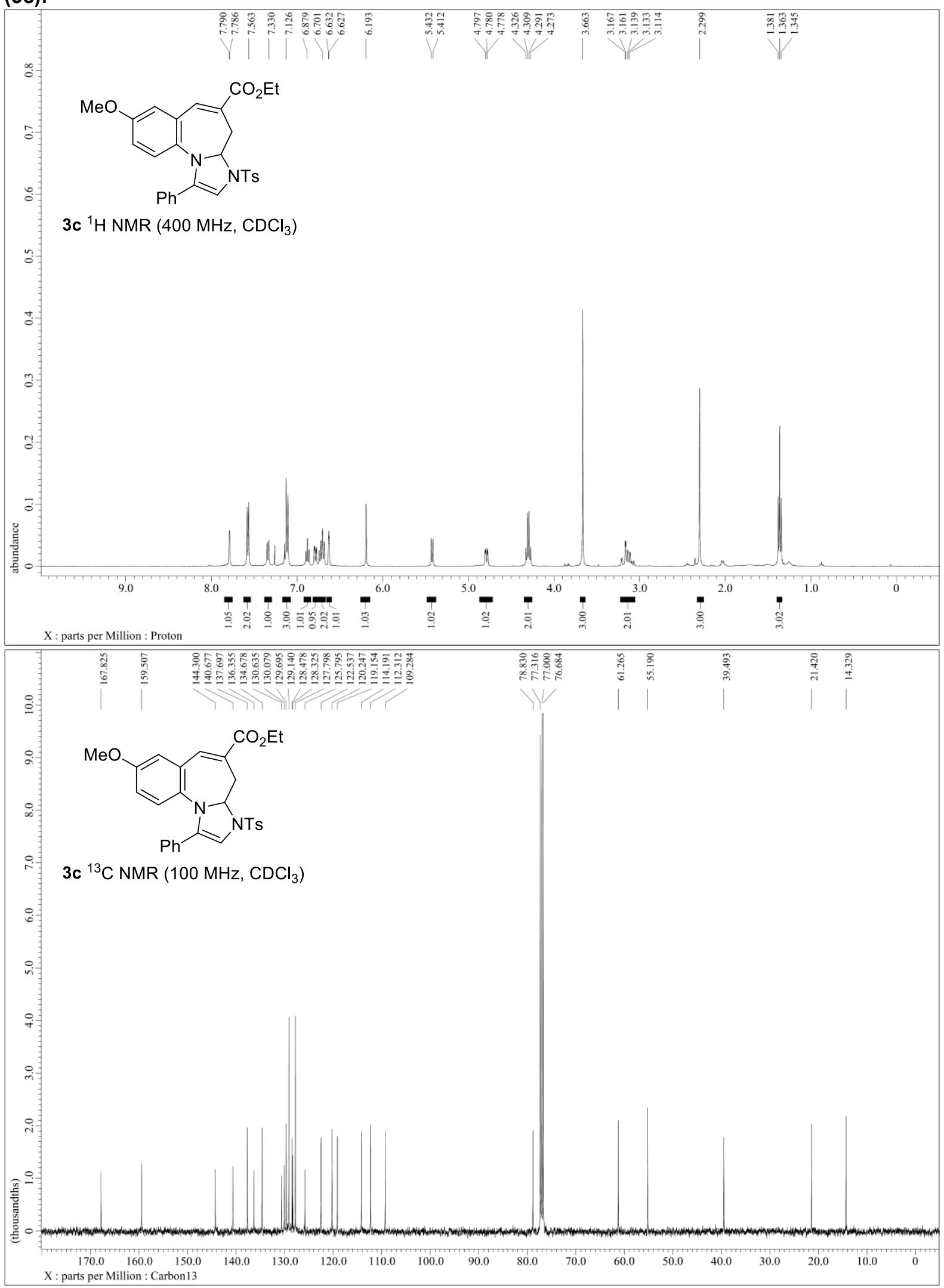




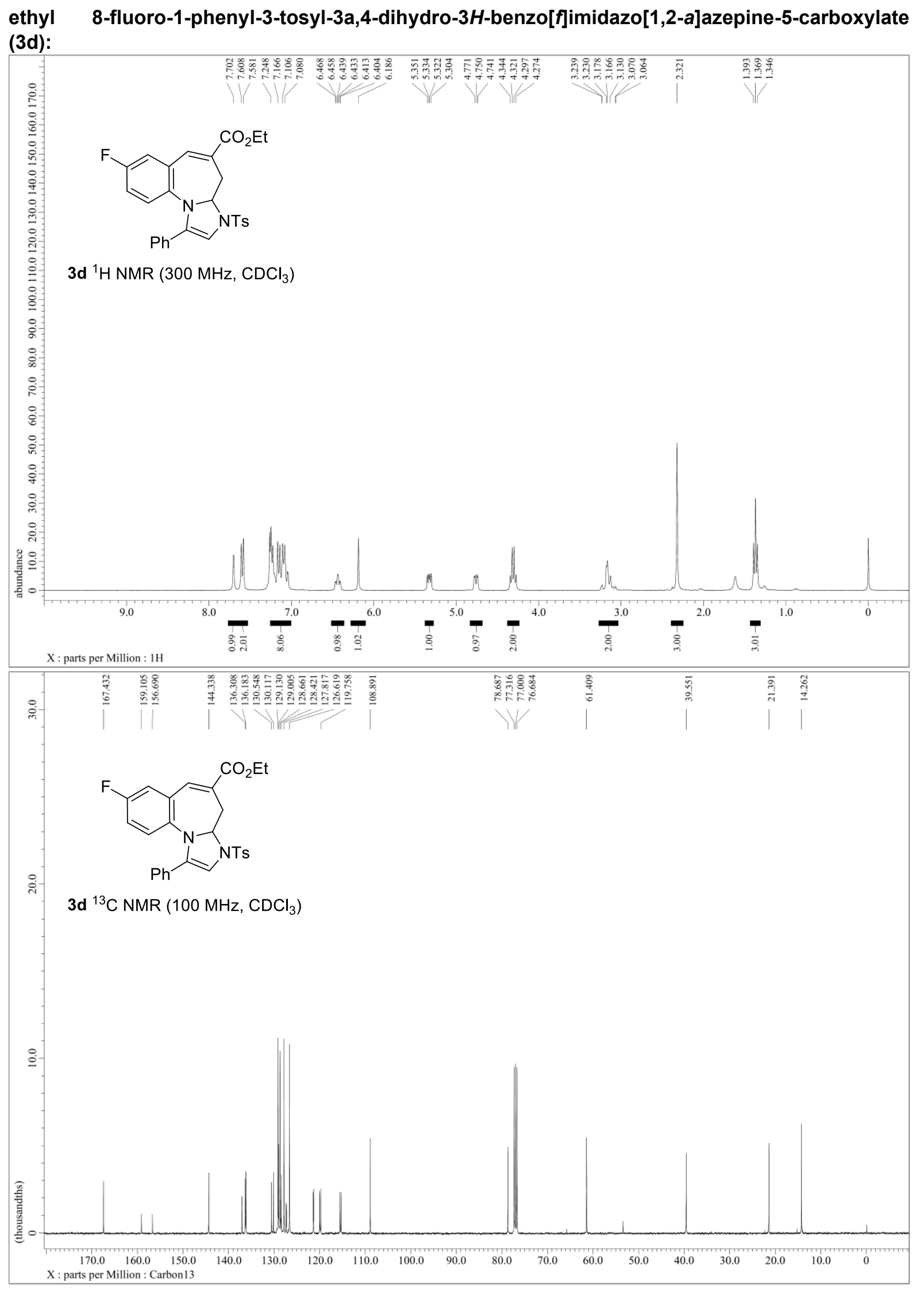

20 
ethyl 10-methoxy-1-phenyl-4H-benzo[f]imidazo[1,2-a]azepine-5-carboxylate (3e'):
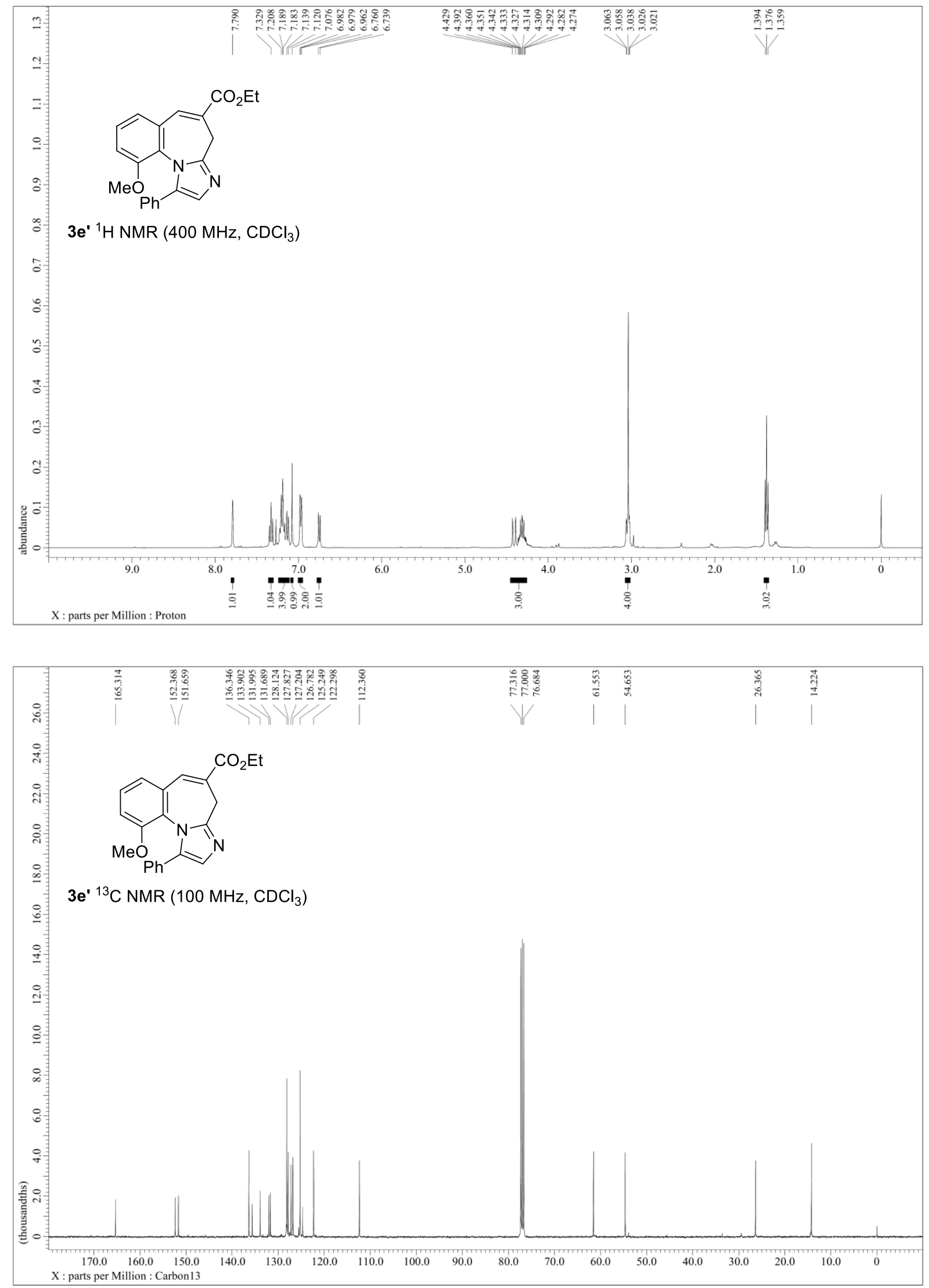
ethyl 6-phenyl-4-tosyl-3a,4-dihydro-3H-imidazo[1',2':1,7]azepino[2,3-h]quinoline-2-carboxylate

(3f):

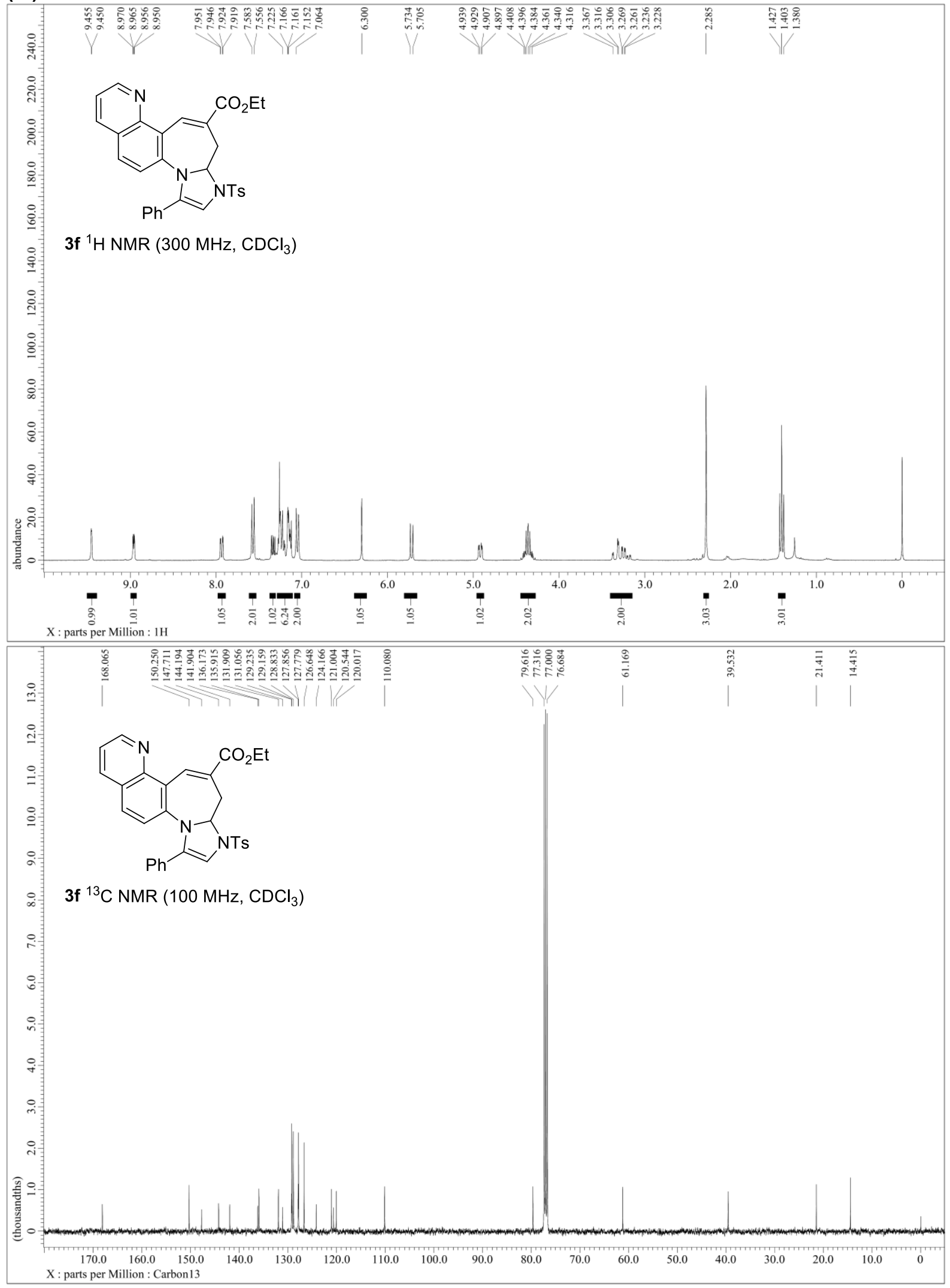

22 
carboxylate $(3 \mathrm{~g})$ :

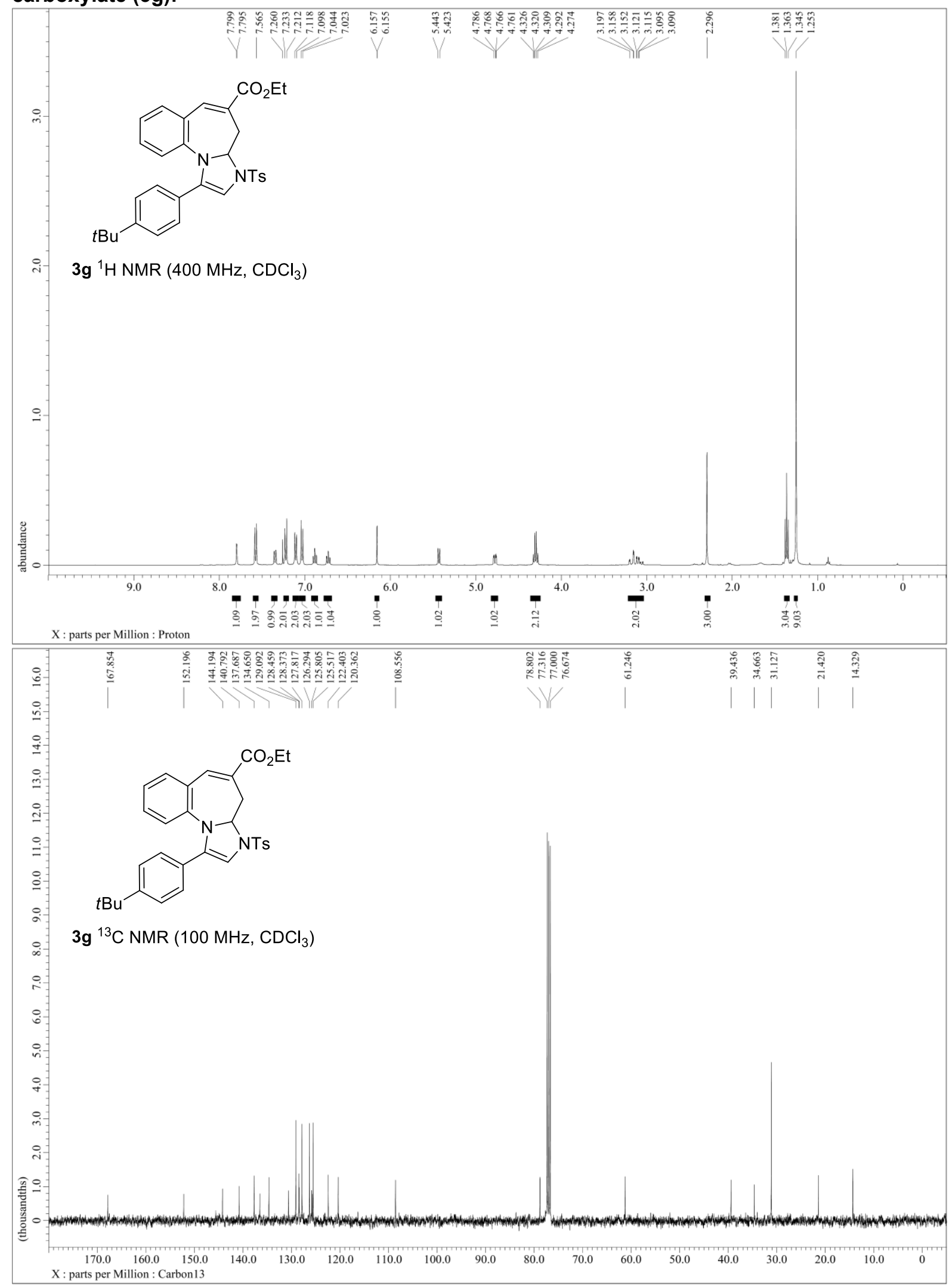


ethyl 3-tosyl-1-(4-(trifluoromethyl)phenyl)-3a,4-dihydro-3H-benzo[f]imidazo[1,2-a]azepine-5carboxylate (3h):

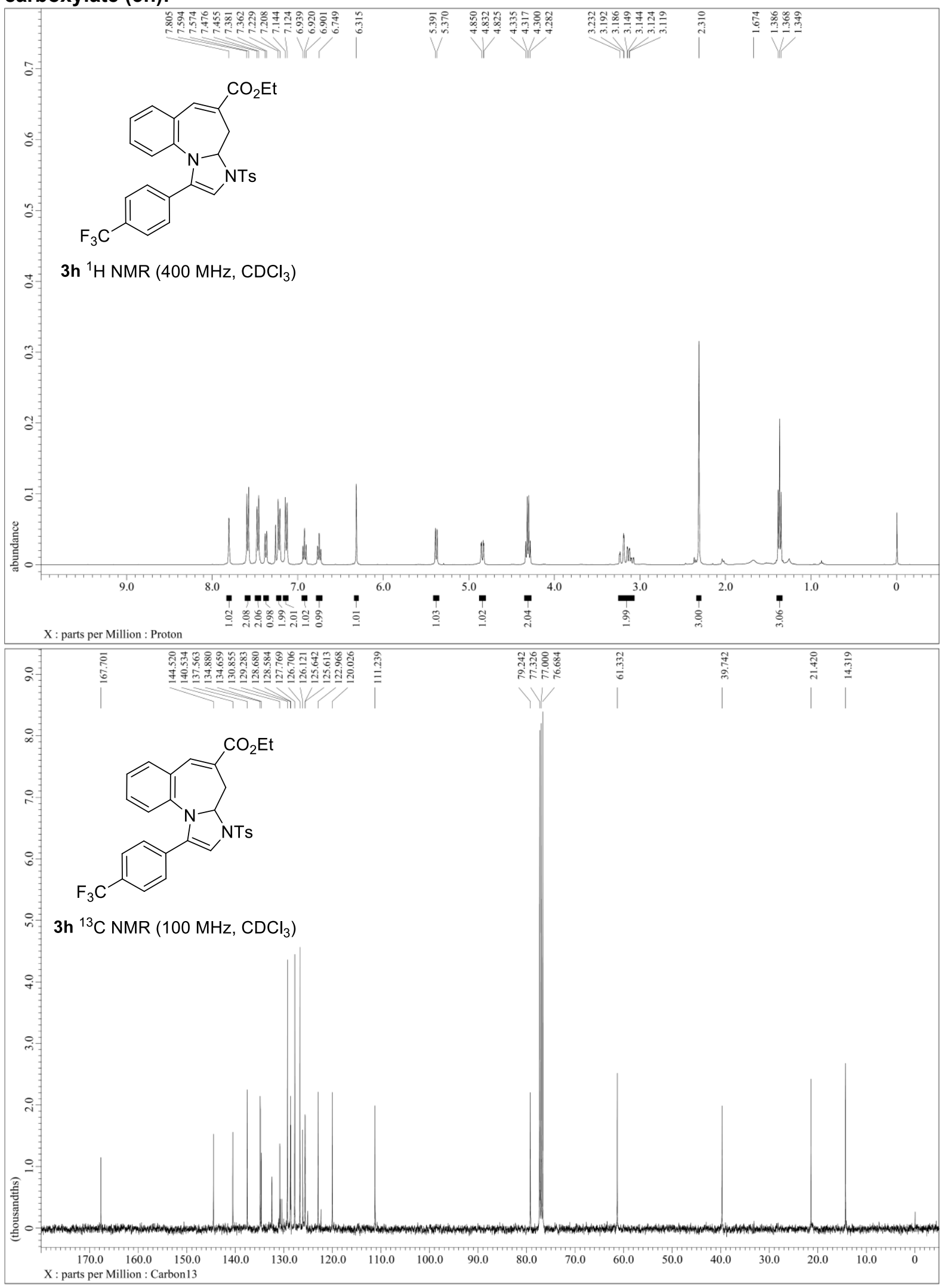


ethyl 1-(3-methoxyphenyl)-3-tosyl-3a,4-dihydro-3H-benzo[f]imidazo[1,2-a]azepine-5-carboxylate (3i):

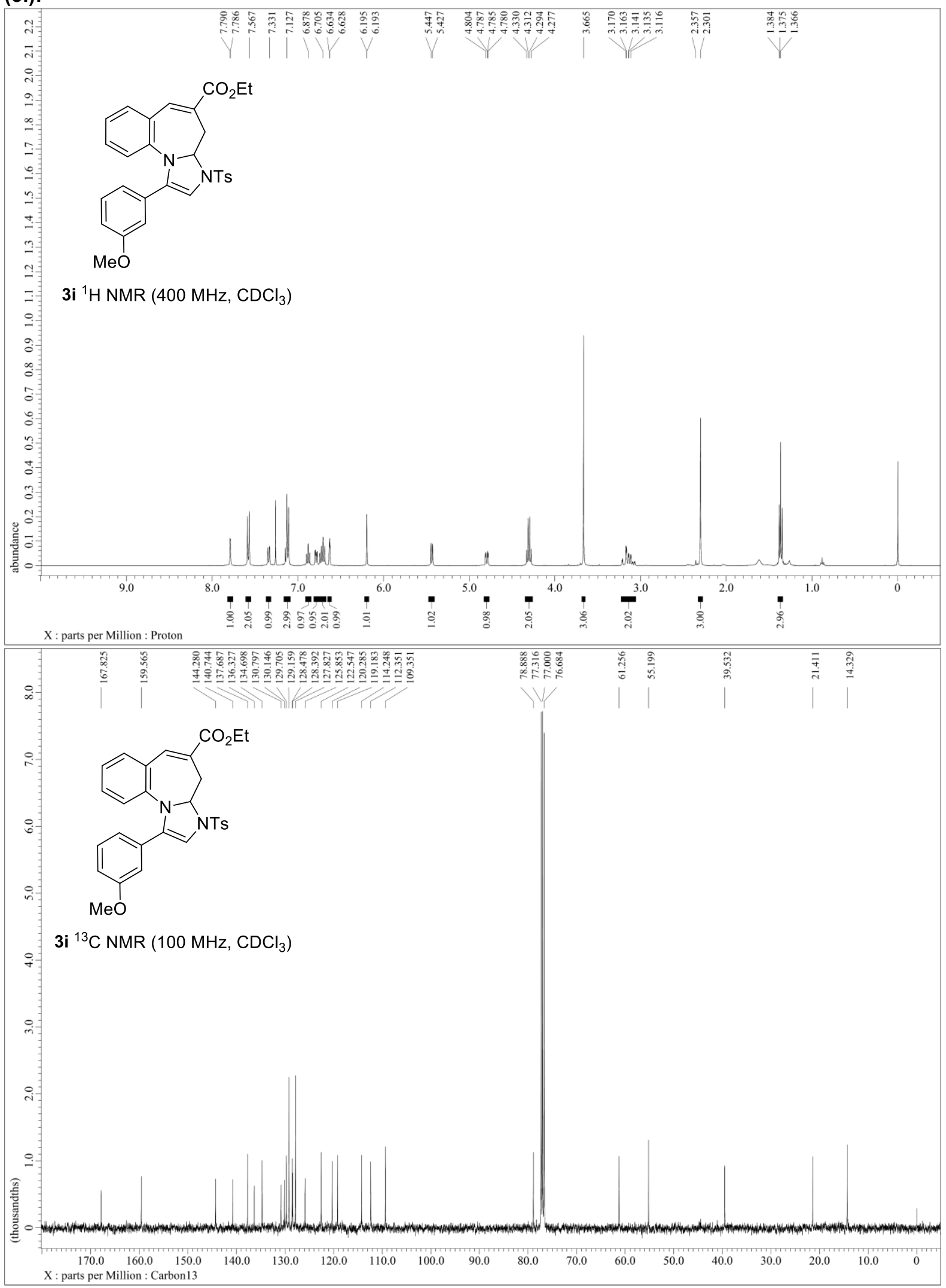


ethyl 1-(3-fluorophenyl)-3-tosyl-3a,4-dihydro-3H-benzo[f]imidazo[1,2-a]azepine-5-carboxylate (3j):

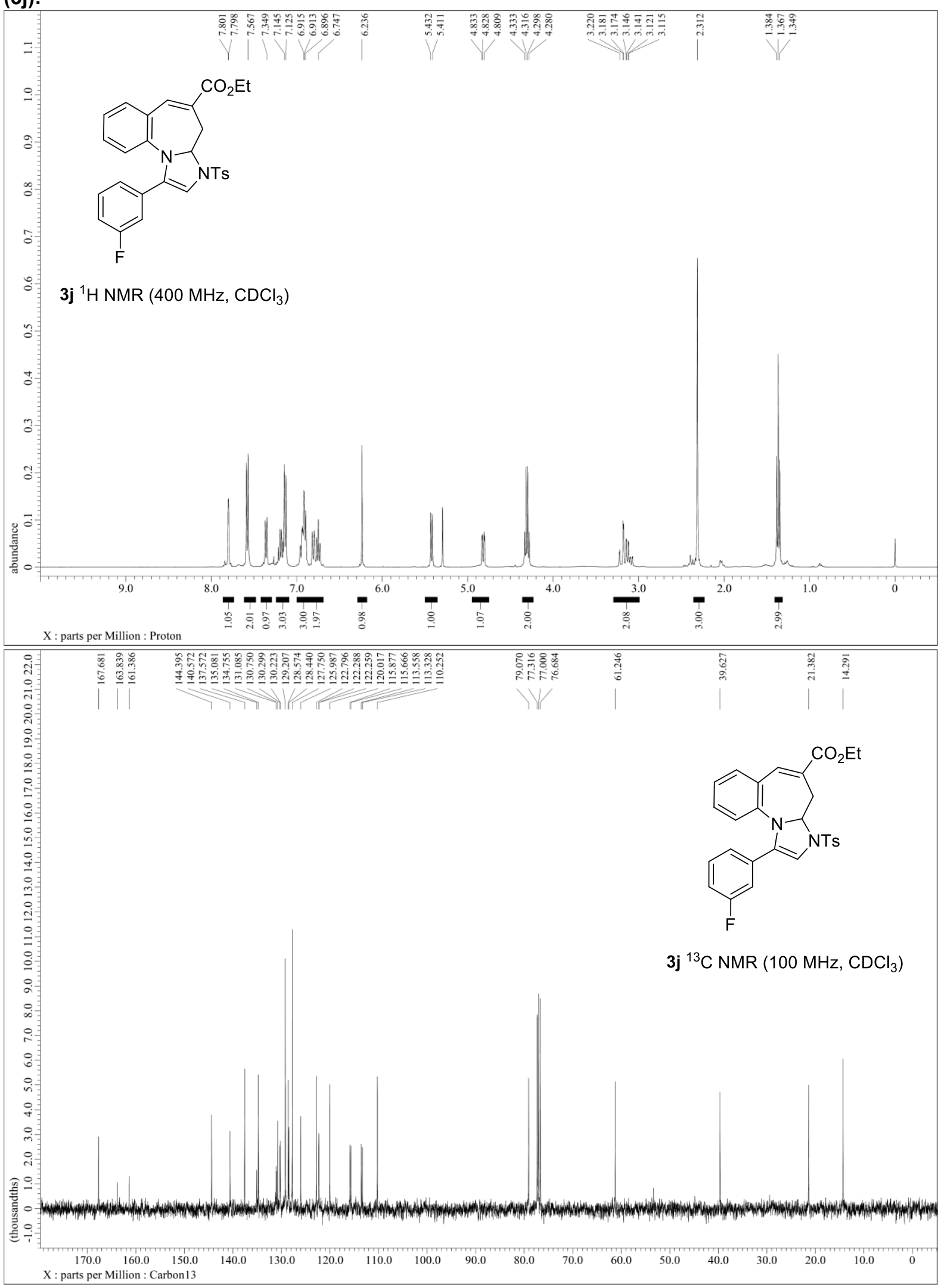


ethyl 1-(2-fluorophenyl)-3-tosyl-3a,4-dihydro-3H-benzo[f]imidazo[1,2-a]azepine-5-carboxylate (3k):

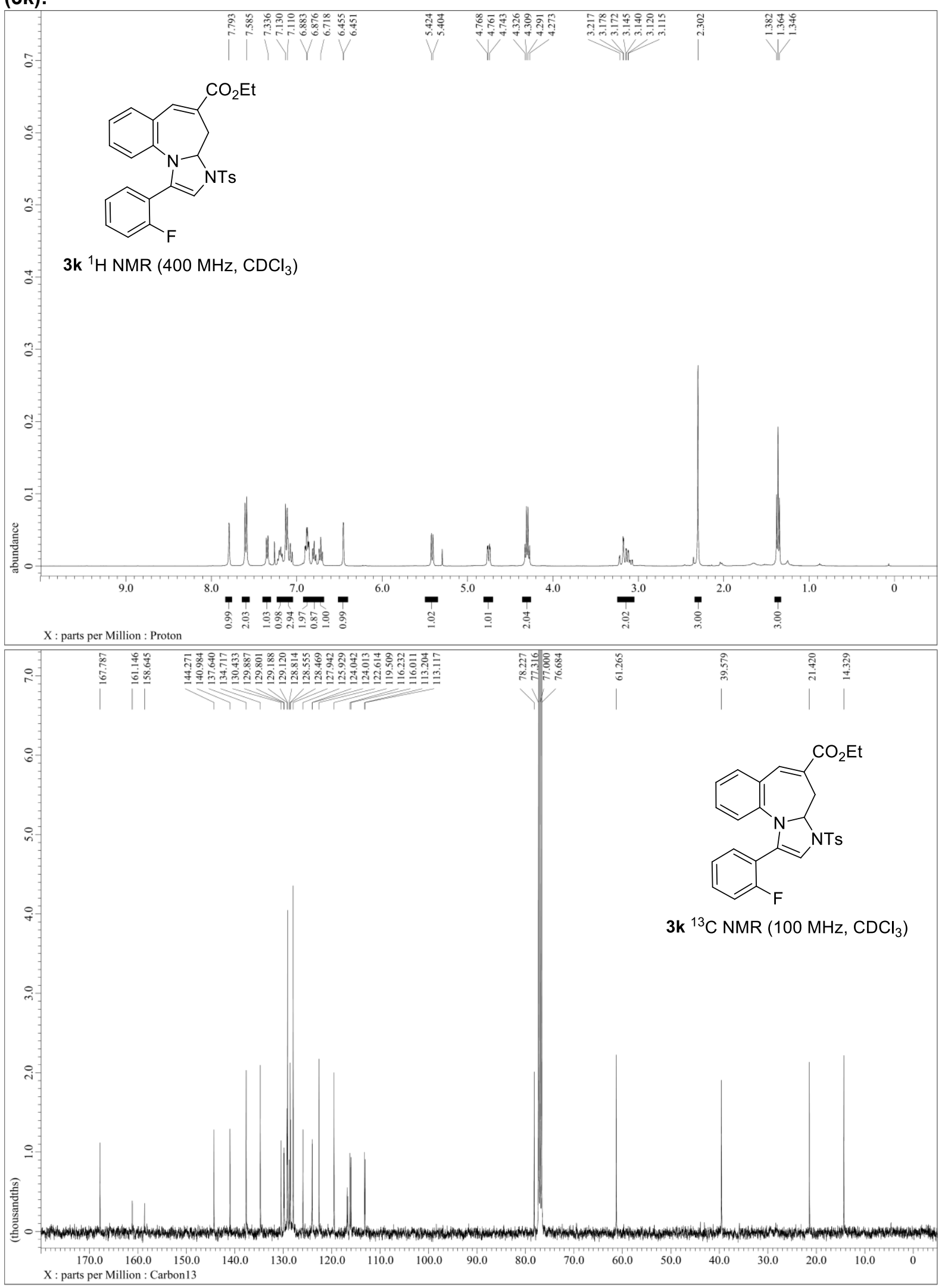


ethyl 1-(3,5-difluorophenyl)-3-tosyl-3a,4-dihydro-3H-benzo[f]imidazo[1,2-a]azepine -5 -carboxylate (3I):

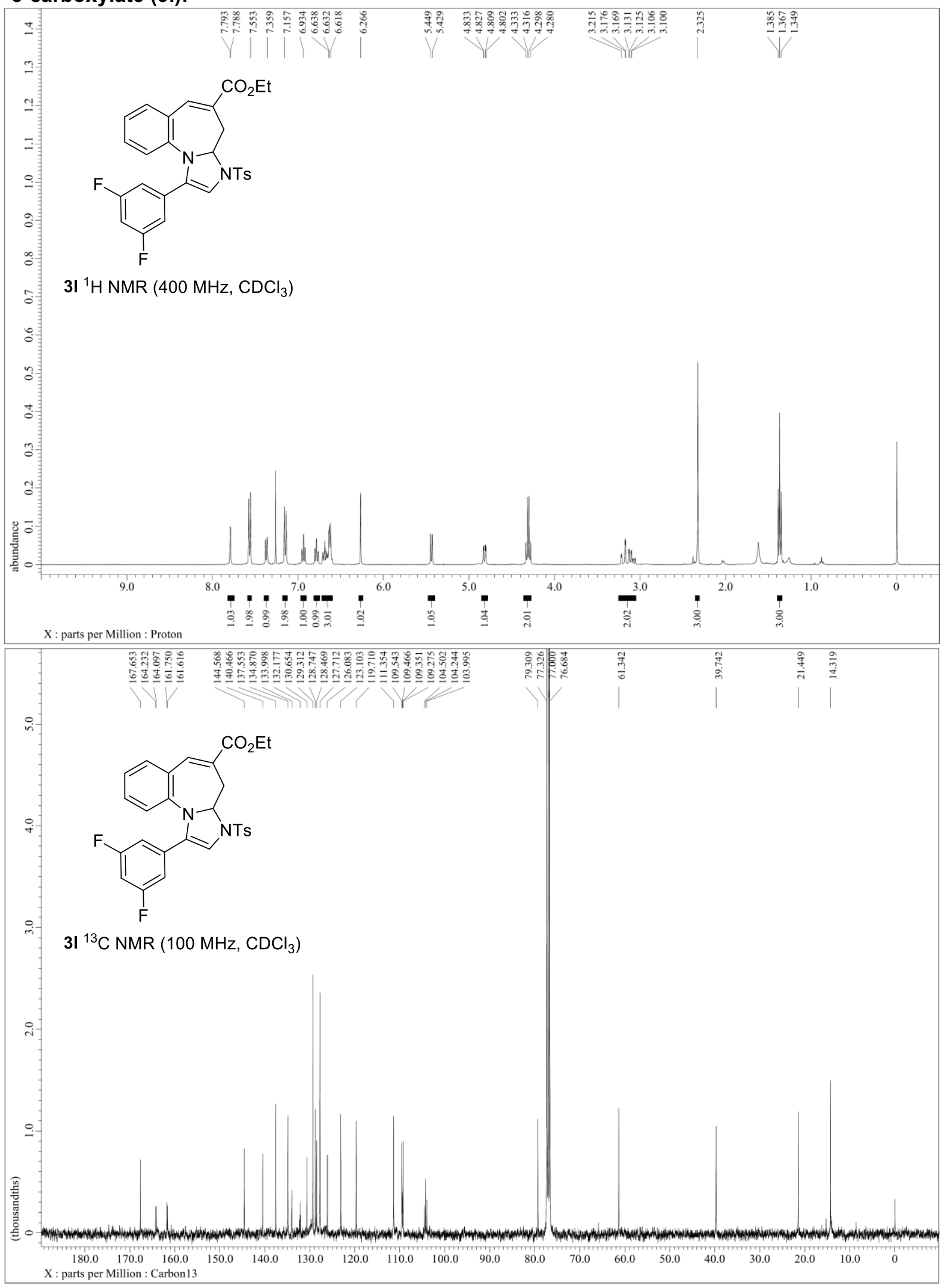


ethyl 1-phenyl-3-(phenylsulfonyl)-3a,4-dihydro-3H-benzo[f]imidazo[1,2-a]azepine-5-carboxylate $(3 \mathrm{~m})$ :

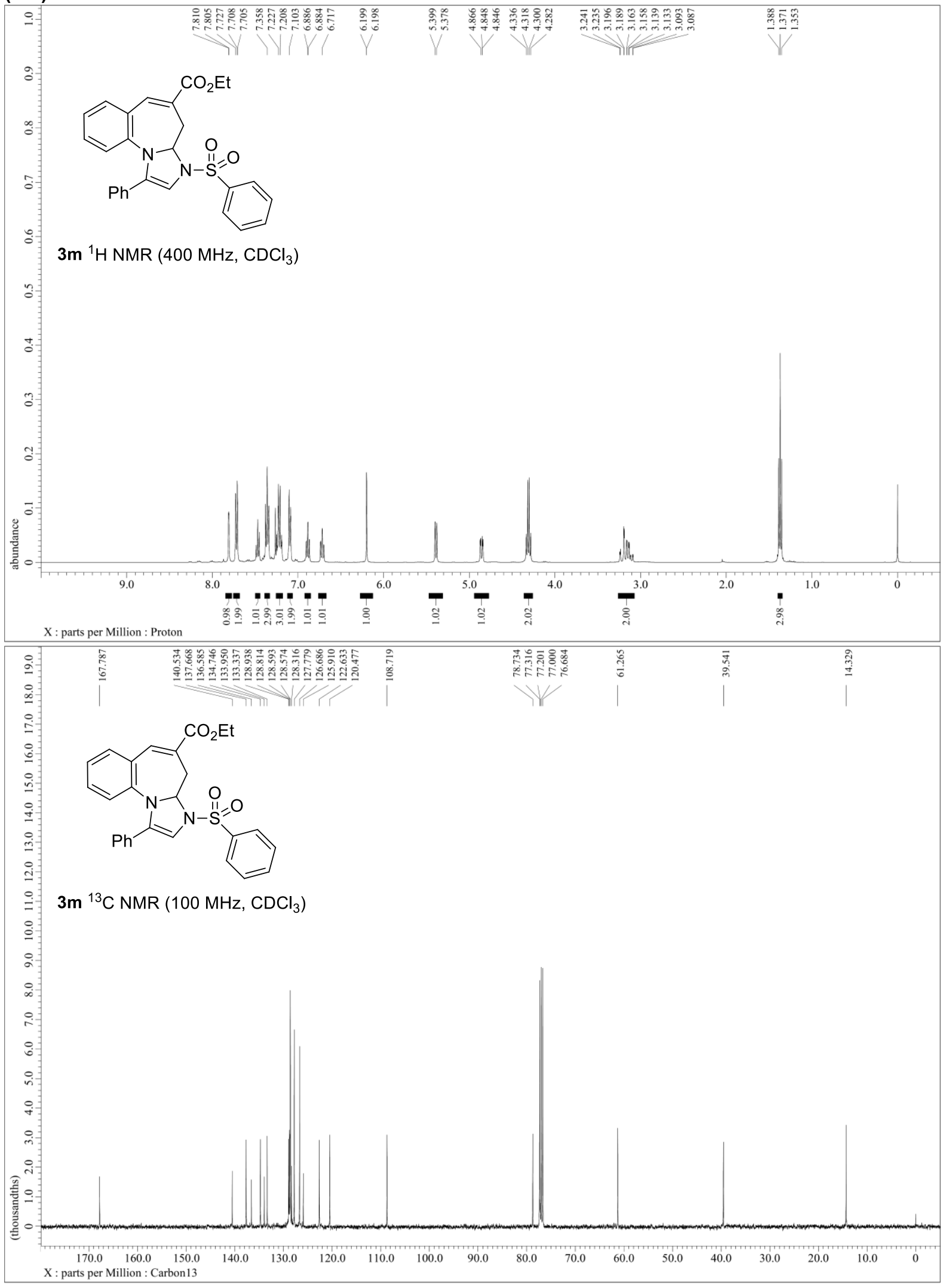


ethyl 3-((4-methoxyphenyl)sulfonyl)-1-phenyl-3a,4-dihydro-3H-benzo[f $]$ imidazo [1,2-a]azepine-5-carboxylate $(3 n)$ :

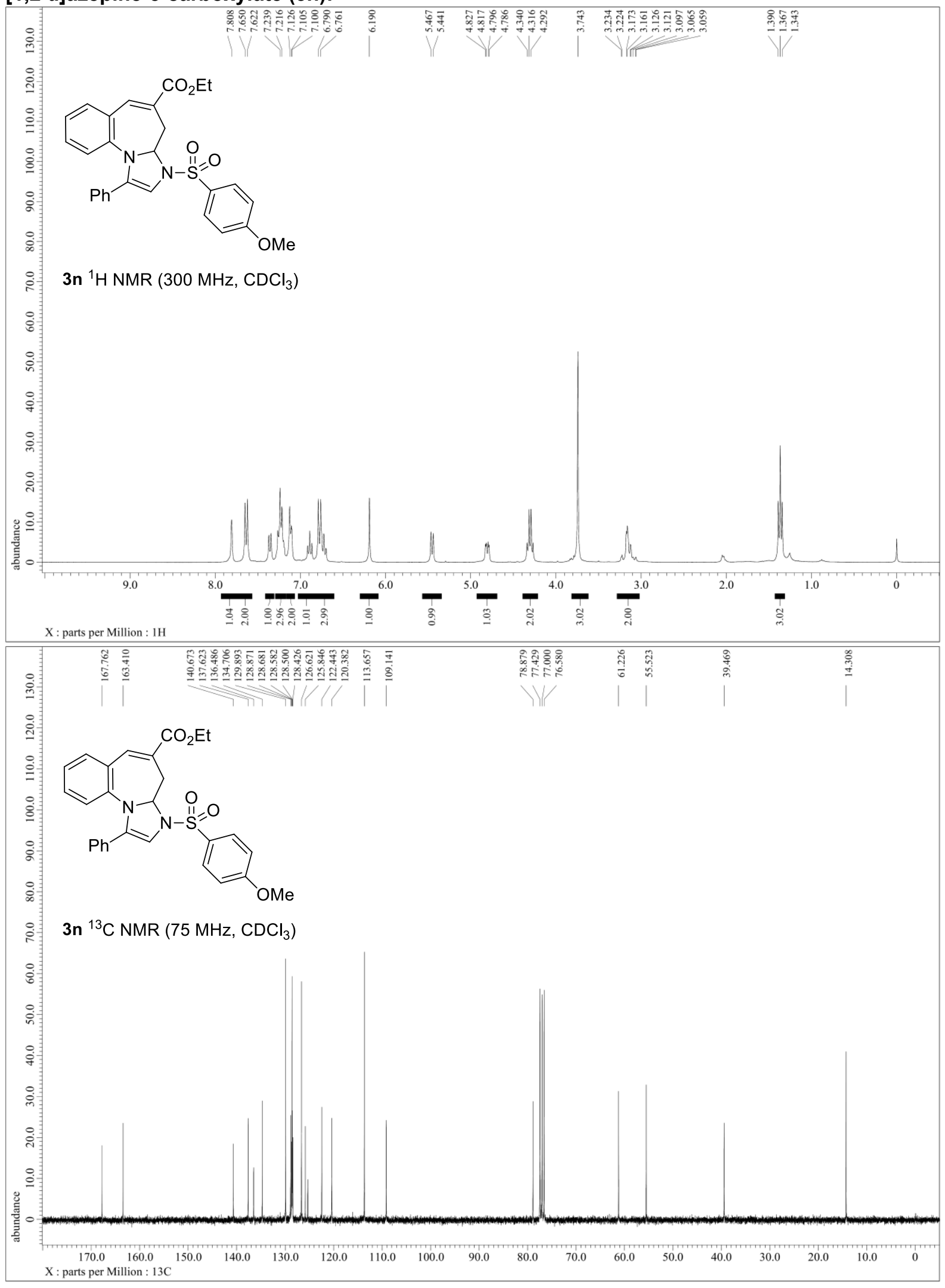


ethyl 1-phenyl-3-((4-(trifluoromethyl)phenyl)sulfonyl)-3a,4-dihydro-3H-benzo[f]imidazo[1,2a]azepine-5-carboxylate (30):

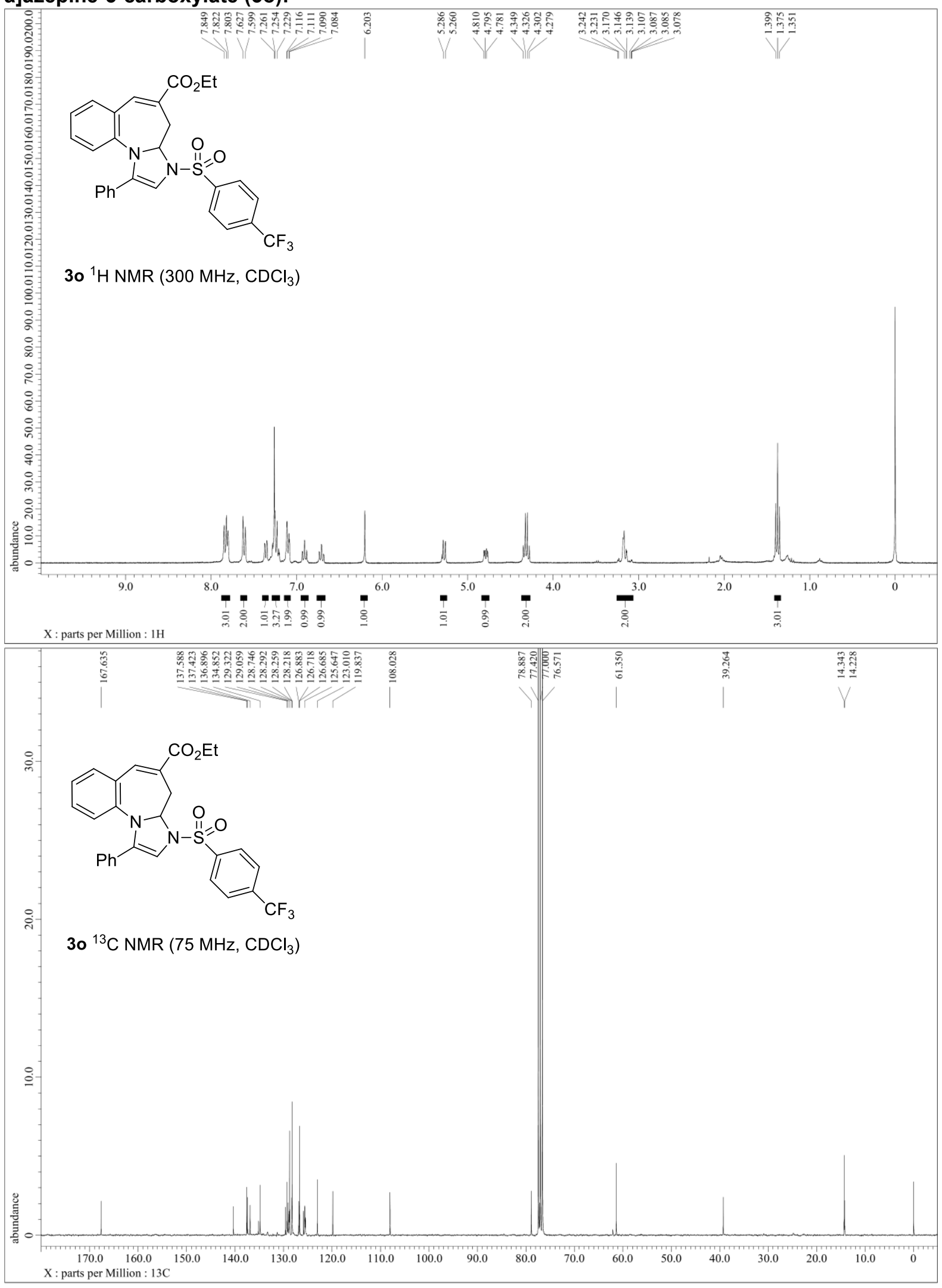




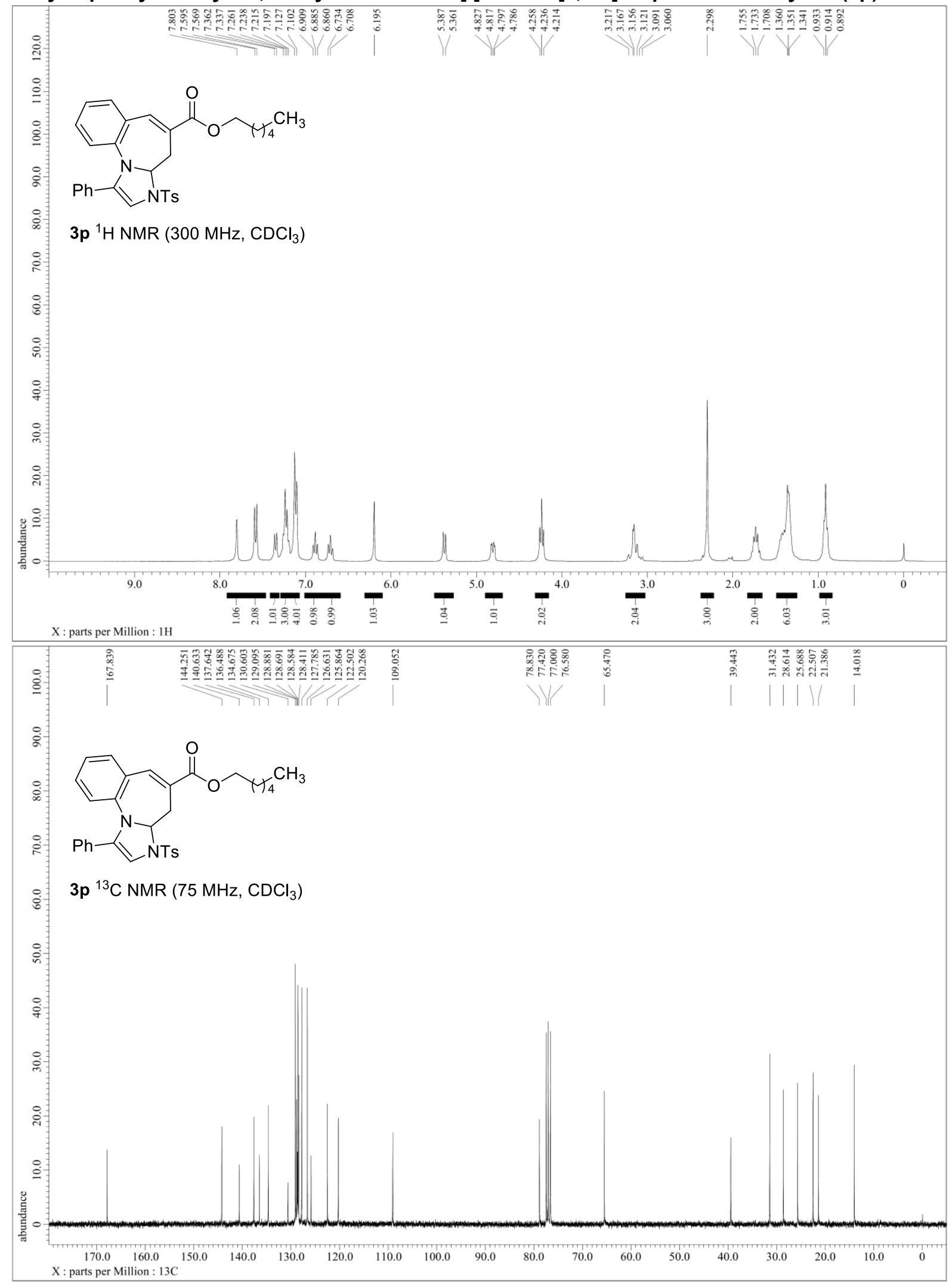




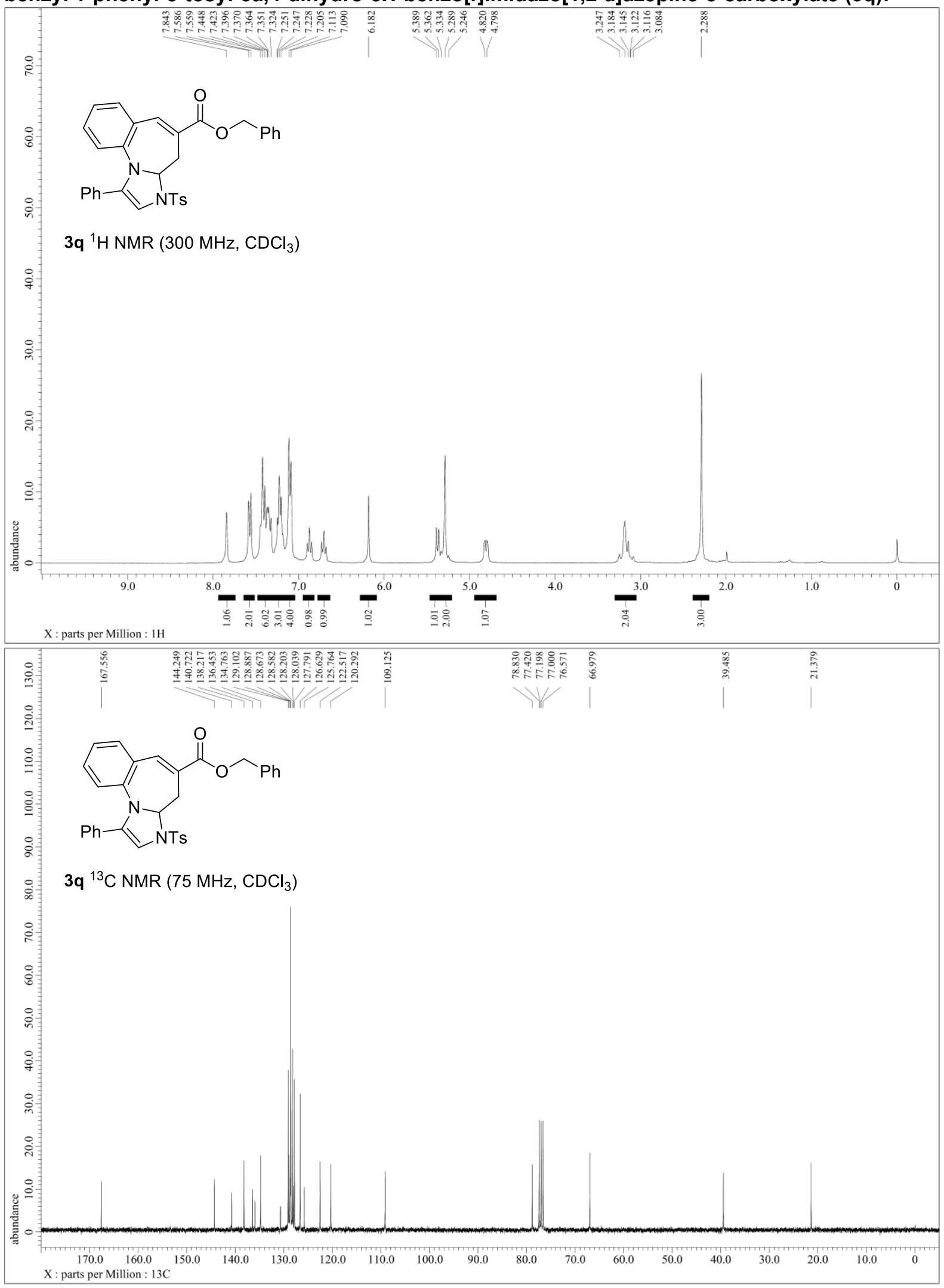


isopropyl 1-phenyl-3-tosyl-3a,4-dihydro-3H-benzo[f]imidazo[1,2-a]azepine-5-carboxylate (3r):

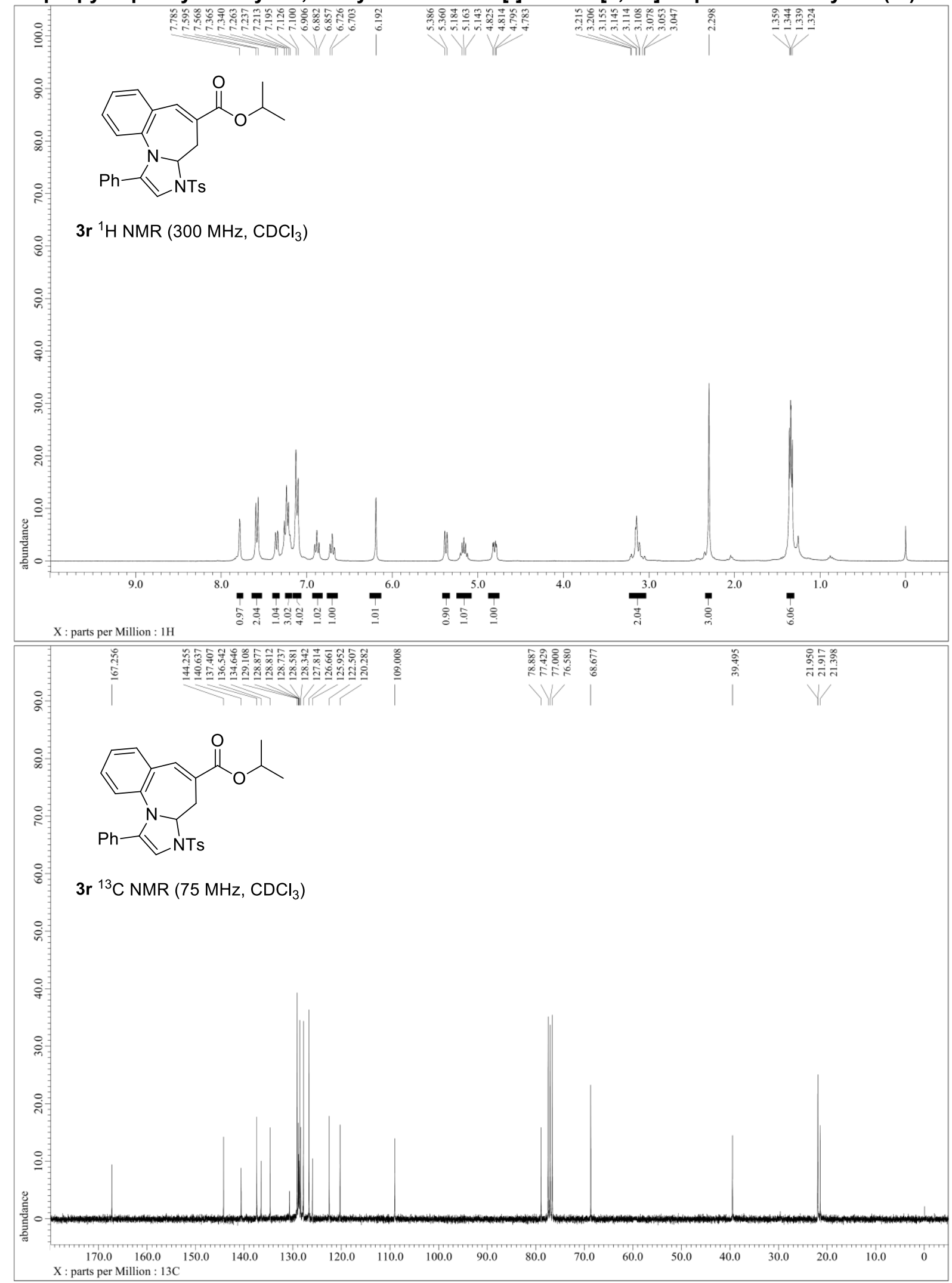


cyclohexyl 1-phenyl-3-tosyl-3a,4-dihydro-3H-benzo[f]imidazo[1,2-a]azepine-5-carboxylate (3s):

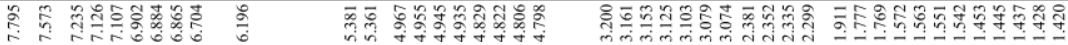<smiles>O=C(OC1CCCCC1)C1=Cc2ccccc2N2C(c3ccccc3)=C[NH+](S)C2C1</smiles>

3. 3s ${ }^{1} \mathrm{H} \mathrm{NMR}\left(400 \mathrm{MHz}, \mathrm{CDCl}_{3}\right)$

공

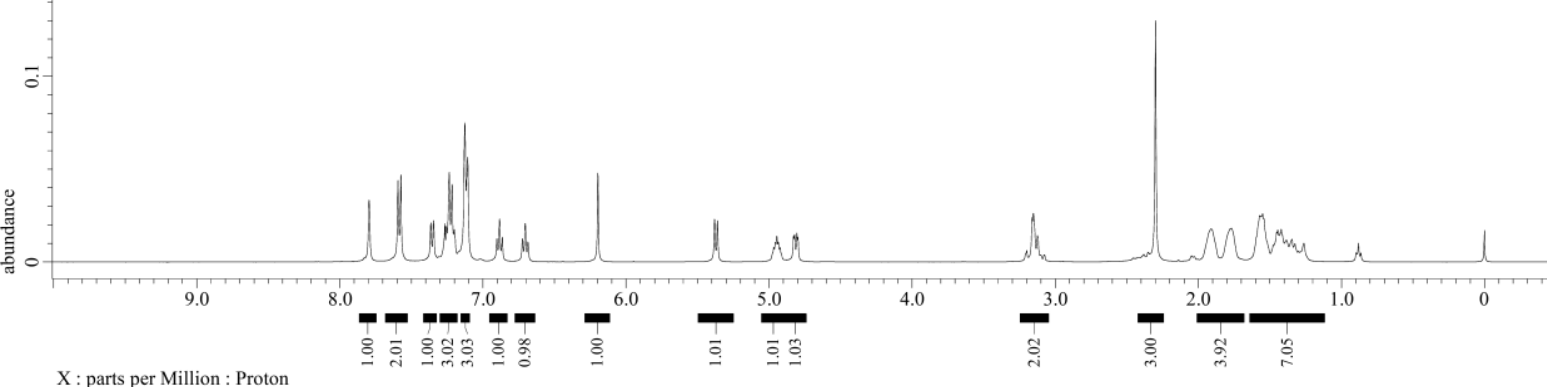

X : parts per Million : Proton

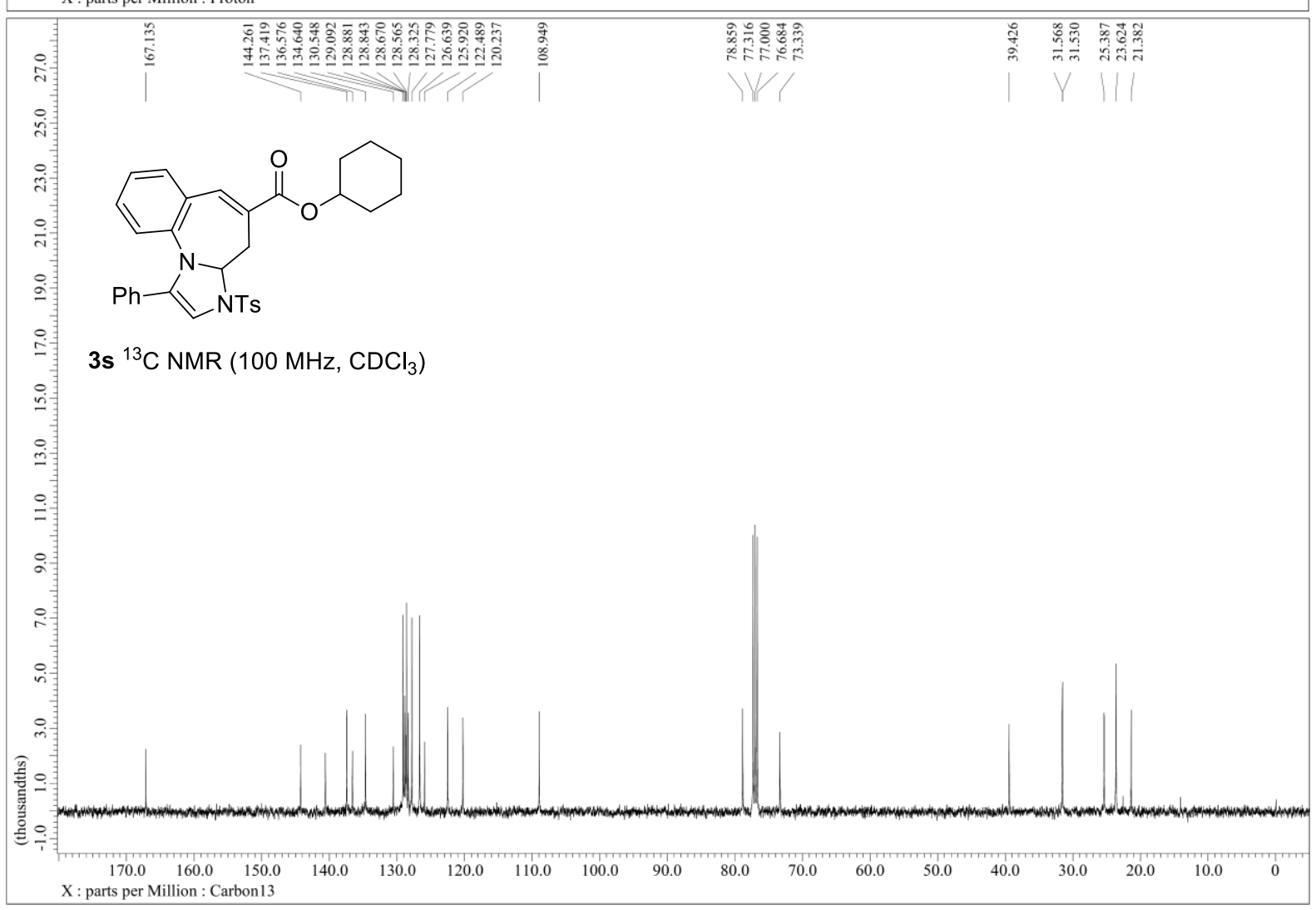


tert-butyl 1-phenyl-3-tosyl-3a,4-dihydro-3H-benzo[f]imidazo[1,2-a]azepine-5-carboxylate (3t):
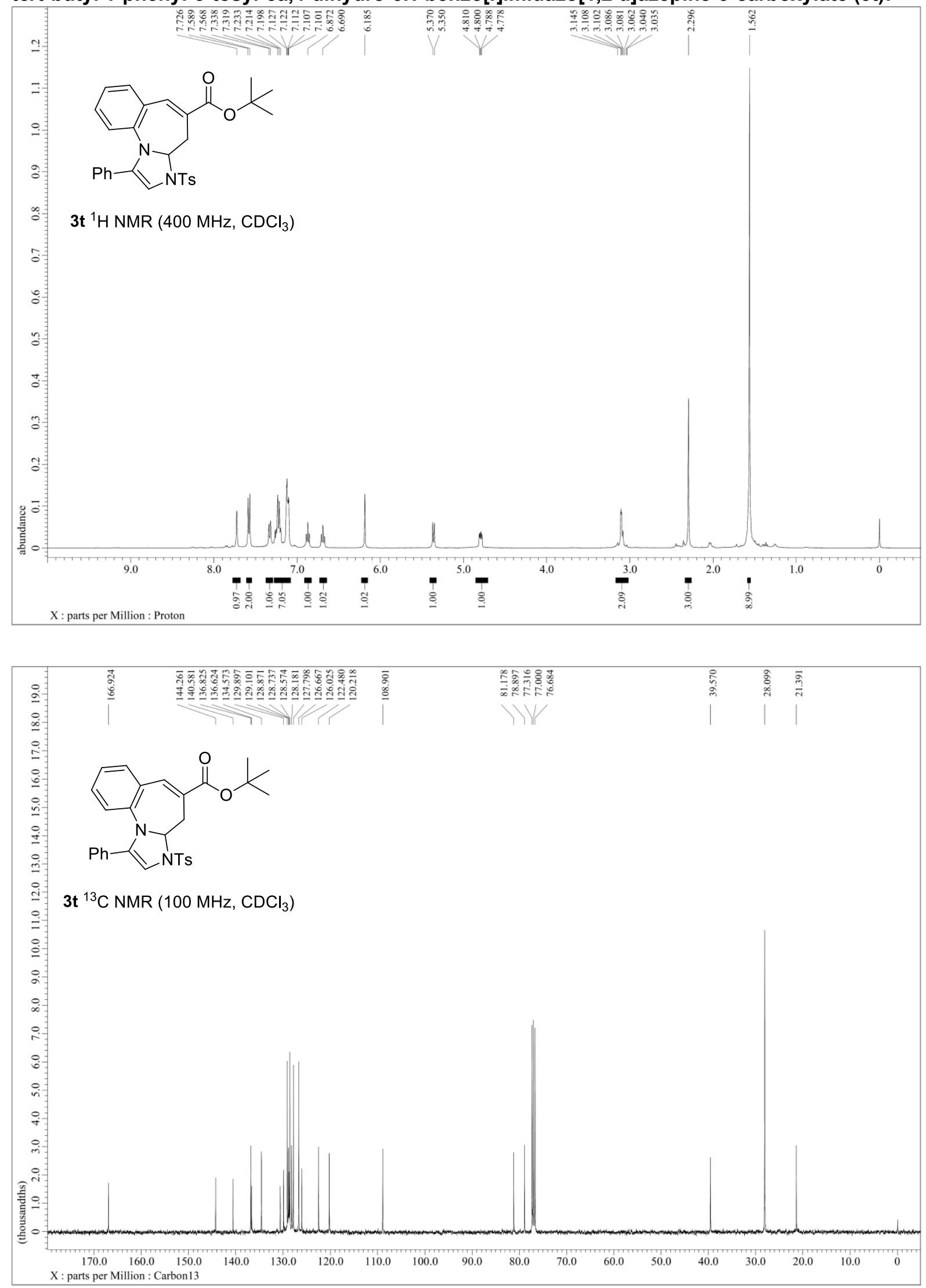
4-methoxyphenyl 1-phenyl-3-tosyl-3a,4-dihydro-3H-benzo[f]imidazo[1,2-a]azepine-5carboxylate $(3 \mathrm{u})$ :

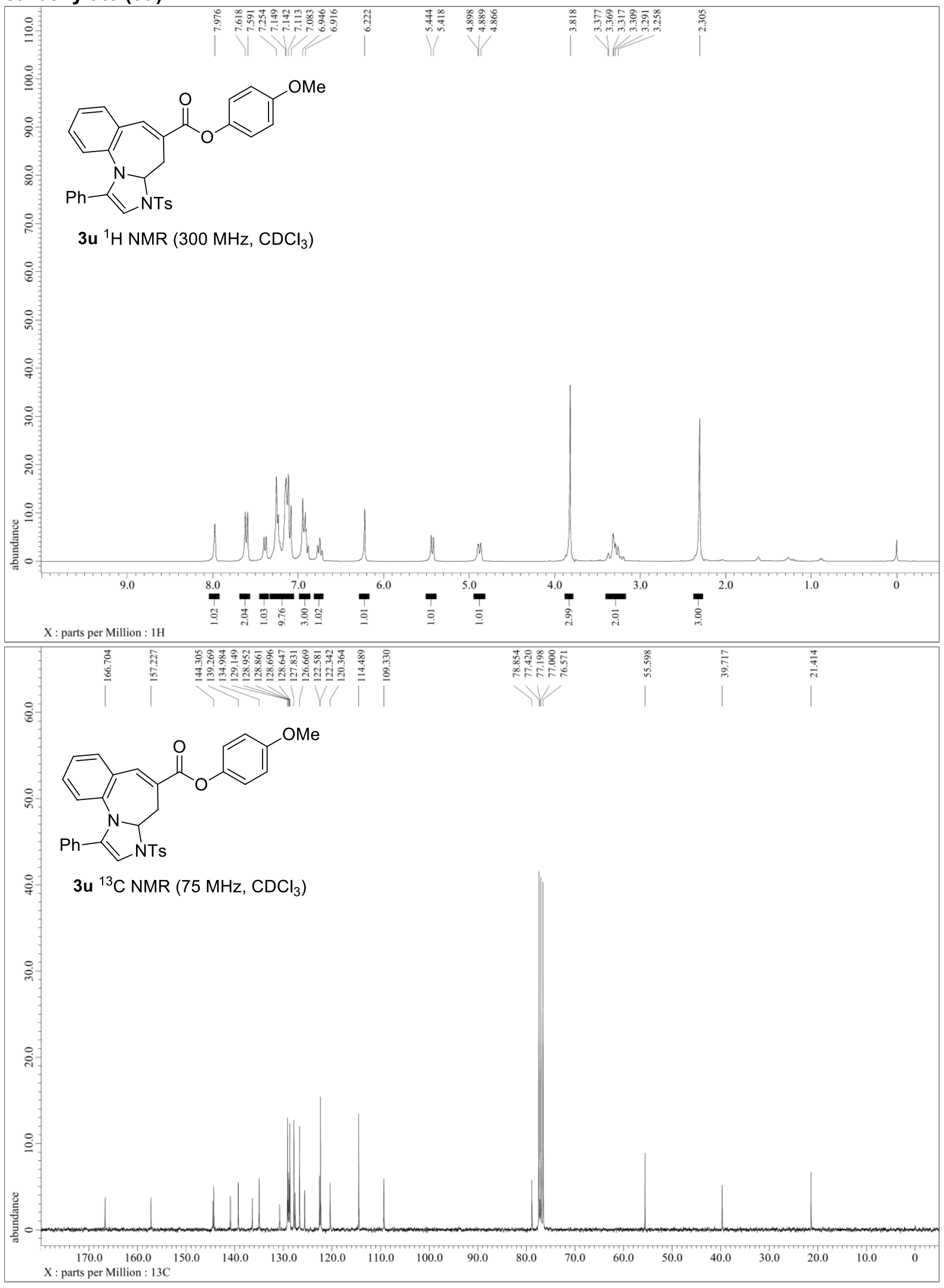


ethyl 1-phenyl-4H-benzo[f]imidazo[1,2-a]azepine-5-carboxylate (4):

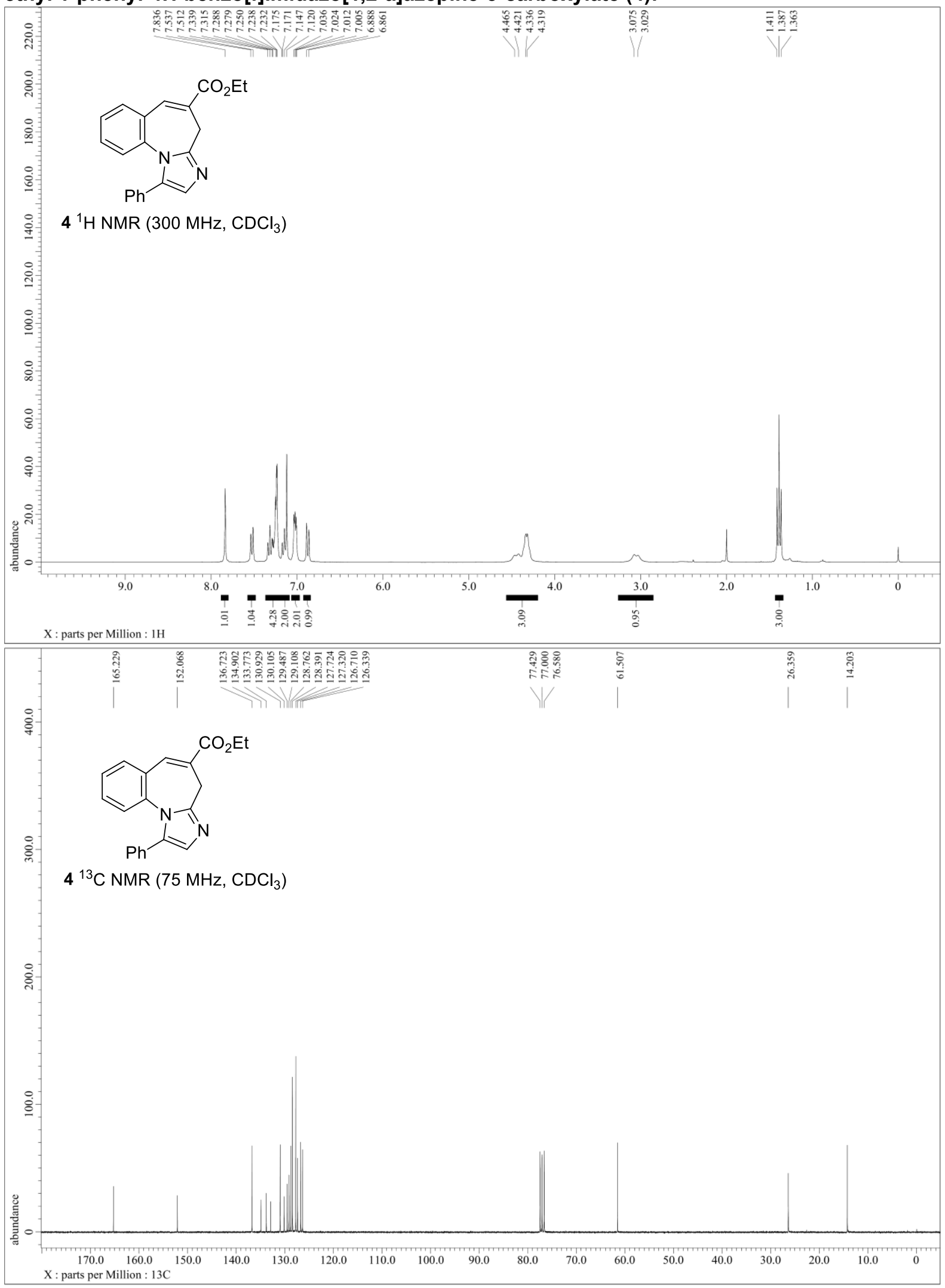


ethyl 1-phenyl-5,6-dihydro-4H-benzo[f]imidazo[1,2-a]azepine-5-carboxylate (5):

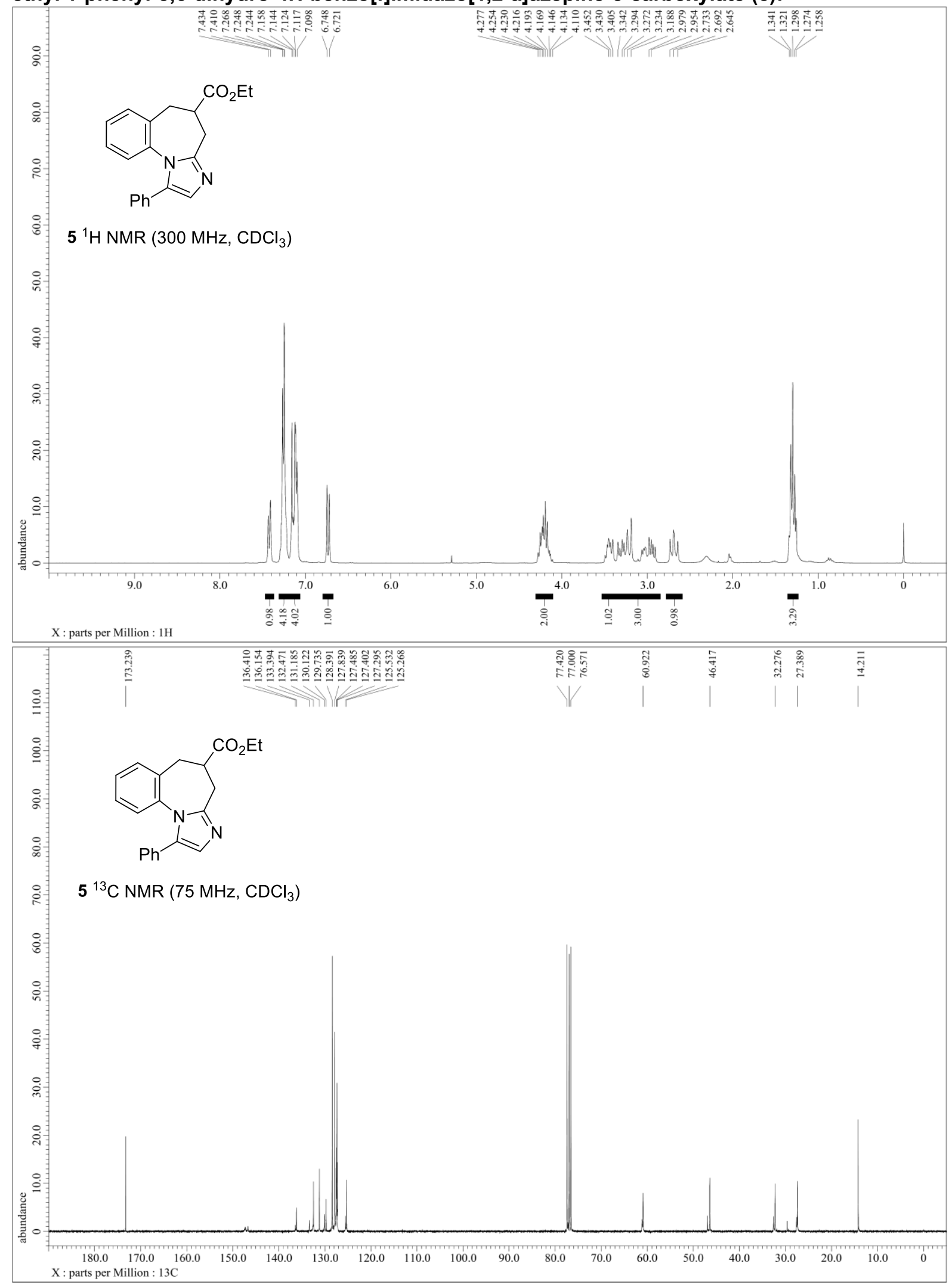


(1-phenyl-4H-benzo[f]imidazo[1,2-a]azepin-5-yl)methanol (6):

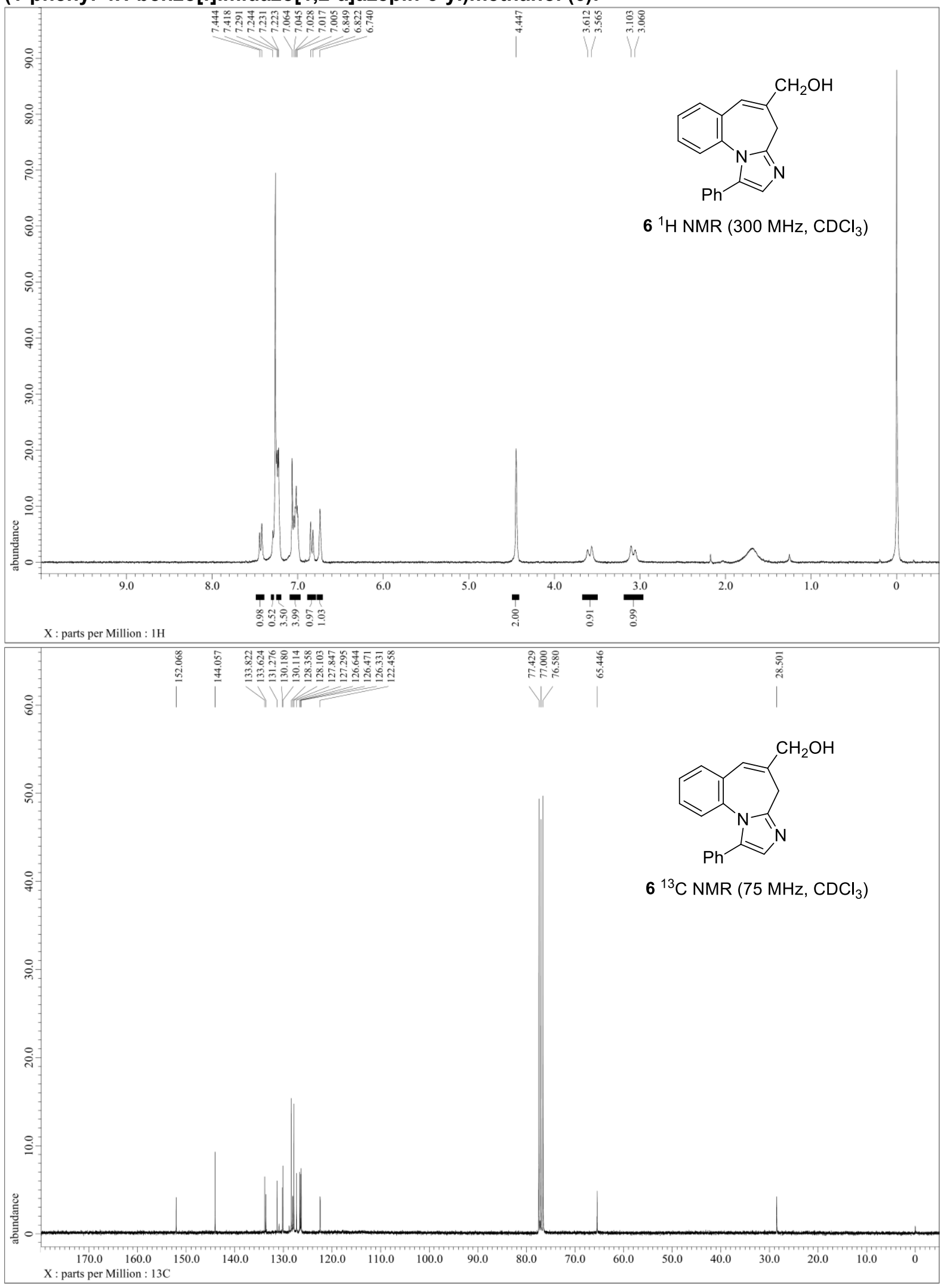


5-(chloromethyl)-1-phenyl-4H-benzo[f]imidazo[1,2-a]azepine (7):

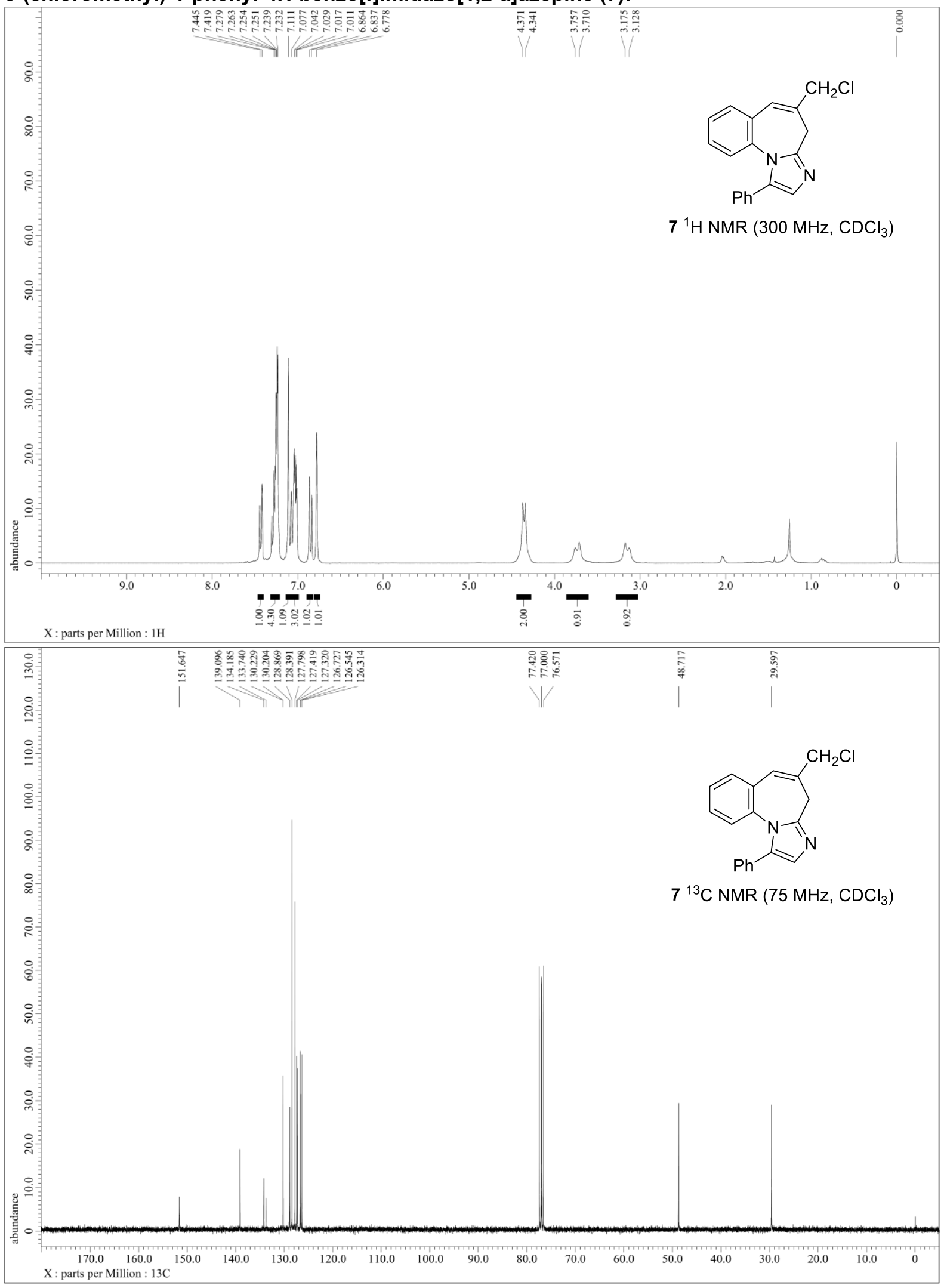


ethyl 3a-deuterium-1-phenyl-3-tosyl-3a,4-dihydro-3H-benzo[f]imidazo[1,2-a]azepine-5carboxylate (3a $\left.{ }^{1}-D\right)$ :
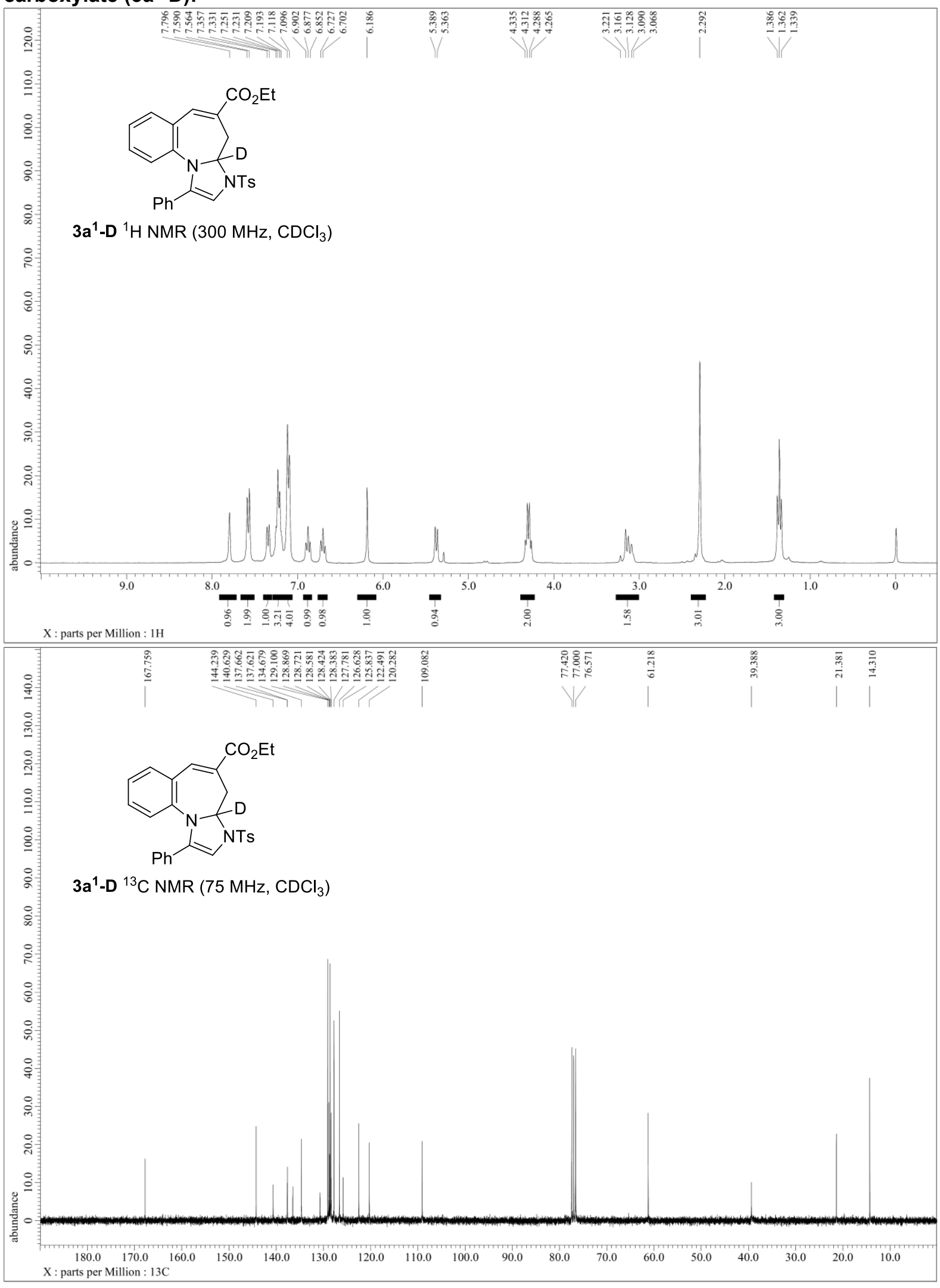
ylidene)acetate (9):
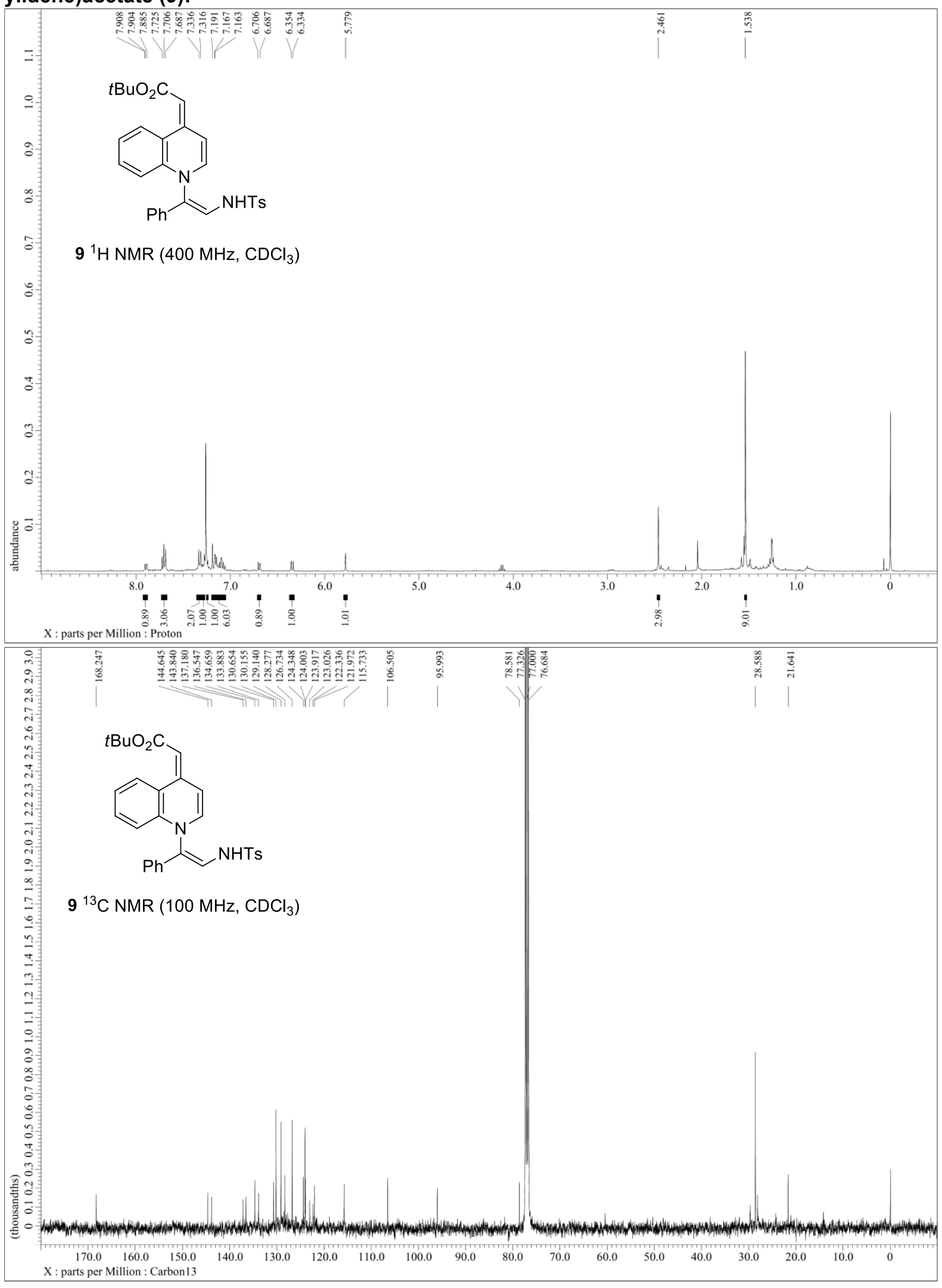